\title{
Gravitational waves and geometrical optics in scalar-tensor theories
}

\section{Alice Garoffolo, ${ }^{a, b, c}$ Gianmassimo Tasinato, ${ }^{d}$ Carmelita Carbone, ${ }^{e, f}$ Daniele Bertacca, ${ }^{c, g, h}$ and Sabino Matarrese ${ }^{c, g, h, i}$}

\author{
${ }^{a}$ Institute Lorentz, Leiden University, PO Box 9506, Leiden 2300 RA, The Netherlands \\ ${ }^{b}$ Dipartimento di Fisica "Aldo Pontremoli", Università degli Studi di Milano, via Celoria 16, \\ I-20133 Milano, Italy \\ ${ }^{c}$ Dipartimento di Fisica e Astronomia G. Galilei, Università degli Studi di Padova, I-35131 \\ Padova, Italy. \\ ${ }^{d}$ Department of Physics, Swansea University, Swansea, SA2 8PP, UK \\ ${ }^{e}$ INAF - Istituto di Astrofisica Spaziale e Fisica cosmica di Milano, via Alfonso Corti 12, \\ 20133, Milano, Italy \\ ${ }^{f}$ INFN, Sezione di Milano, via Celoria 16, I-20133 Milano, Italy \\ ${ }^{g}$ INFN, Sezione di Padova, via F. Marzolo 8, I-35131, I-35131 Padova, Italy. \\ ${ }^{h}$ INAF - Osservatorio Astronomico di Padova, vicolo dell'Osservatorio 5, I-35122 Padova, \\ Italy. \\ ${ }^{i}$ Gran Sasso Science Institute, viale F. Crispi 7, I-67100 L'Aquila, Italy. \\ E-mail: garoffolo@lorentz.leidenuniv.nl,g.tasinato2208gmail.com, \\ carmelita.carbone@inaf.it, daniele.bertacca@pd.infn.it, \\ sabino.matarrese@pd.infn.it
}

\begin{abstract}
The detection of gravitational waves (GWs) propagating through cosmic structures can provide invaluable information on the geometry and content of our Universe as well as on the fundamental theory of gravity. In order to test possible departures from General Relativity, it is essential to analyze, in a modified gravity setting, how GWs propagate through a perturbed cosmological space-time. Working within the framework of geometrical optics, we develop tools to address this topic for a broad class of scalar-tensor theories, including scenarios with non-minimal, derivative couplings between scalar and tensor modes. By focussing on a set-up where scalar modes propagate with the same speed as tensor degrees of freedom, we determine the corresponding evolution equations for the GW amplitude and polarization tensor. The former satisfies a generalized evolution equation that includes possible effects due to a variation of the effective Planck scale; the latter can fail to be parallel transported along the GW geodesics unless certain conditions are satisfied. We apply our general formulas to specific scalar-tensor theories with unit tensor speed, and then focus on GW propagation on a perturbed space-time. We determine corrections to standard formulas for the GW luminosity distance and for the evolution of the polarization tensor, which depend both on modified gravity and on the effects of cosmological perturbations. Our results can constitute a starting point to disentangle among degeneracies from different sectors that can influence GW propagation through cosmological space-times.
\end{abstract}




\section{Contents}

1 Introduction 1

2 General formalism $\quad 4$

2.1 Linearized equations of motion: high and low frequency modes 4

$\begin{array}{lll}2.2 & \text { On the averaging procedure } & 6\end{array}$

2.3 The derivative expansion 6

2.4 On the structure of the energy-momentum tensor $T_{\mu \nu} \quad 8$

2.5 Gauge choice and evolution equations 8

2.5.1 A convenient gauge for theories with a single extra high-frequency mode 8

$\begin{array}{ll}2.5 .2 \text { Compatibility among our gauge choices } & 11\end{array}$

2.5.3 Summary of the gauge conditions and of the relevant equations of motion 12

3 Geometrical optics with a single extra degree of freedom 13

3.1 Ansatz and evolution equations 13

3.2 On the decomposition of the energy-momentum tensor $t_{\mu \nu} \quad 16$

$\begin{array}{lll}3.3 & \text { Separating the spin-2 and spin-0 degrees of freedom } & 17\end{array}$

4 An explicit scalar-tensor realization $\quad 19$

5 GW propagation on a cosmological space-time $\quad 21$

5.1 Cosmic rulers for gravitational waves 23

5.2 Gravitational waves in the observer frame 26

$\begin{array}{lll}\text { 5.2.1 The GW phase } & 26\end{array}$

$\begin{array}{ll}\text { 5.2.2 The GW amplitude } & 28\end{array}$

5.3 Perturbations in the Poisson gauge 30

5.4 The luminosity distance 31

5.5 The polarization tensor $\quad 32$

6 Conclusions 33

A Conditions to ensure a unit speed for scalar fluctuations 35

B Defining spin-2 and spin-0 polarization tensors using tetrads 36

$\begin{array}{lll}\text { B.1 Gauge conditions and null tetrads } & 37\end{array}$

B.2 Decoupling of amplitude's evolution equations 38

\section{Introduction}

The $\Lambda$ CDM model of cosmology (see e.g. [1] for an introduction) offers a well defined, simple framework for analyzing current cosmological data, and it agrees with observations. In this set up, a cosmological constant $\Lambda$ is responsible for the present-day acceleration of our universe. Nevertheless, the extreme smallness of the value of $\Lambda$ with respect to all other energy scales one encounters in particle physics [2], as well as anomalies in observational results (the most recent being the so-called $H_{0}$ tension, see e.g. the assessment in [3]), motivate the exploration of alternative scenarios, possibly based on modifications of General Relativity 
(GR). In modified gravity, additional degrees of freedom are introduced, whose dynamics drive present-day cosmological acceleration (see e.g. [4-6] for reviews). The minimal possibility for modifying gravity is to consider scalar-tensor theories, where a single scalar field is added to the spin-2 degrees of freedom characterising GR. Thanks to interactions with itself and with spin-2 tensor modes (or matter fields), scalar-tensor theories can exhibit selfaccelerating solutions, where the scalar profile sources accelerating cosmologies with no need of cosmological constant (see e.g. [6] for a review). Moreover, scalar fifth forces can be suppressed thanks to powerful screening mechanisms, which are able to hide the effects of a light scalar nearby sources (see e.g. [7, 8] for reviews on chameleon and Vainshtein screening mechanisms).

The rapidly developing field of gravitational wave cosmology offers new avenues for testing modified theories of gravity (see e.g. the specific analysis of [9-14]). The single event GW170817 [15-18] and its EM counterpart [19] impose strong constraints on modified theories of gravity predicting a speed of gravitational waves different than light [20-23] (see also the analysis in $[24,25]$ ). In general, the physics of standard sirens offer great promises for cosmology [26-33], and studies of cosmological parameter estimation have been carried on for 2nd generation GW experiments (see e.g. [34]), for ET (see [35-37]), for LISA and space-based detectors [38-41]. In the future, GW observables related with GW frequency, chirp mass, and luminosity distance of coalescing events will be able to further constrain (or discover) modified gravity effects with GW observations, see e.g. [41-47] for works discussing the potential of GW cosmology in probing modified gravity.

The purpose of this work is to develop the first steps towards a systematic and consistent approach for studying the propagation of GWs around arbitrary space-times, based on the separation by high and low frequency modes pioneered by Isaacson [48, 49] (see also [50, 51] for early works on the subject). This formalism is at the basis of a geometrical optics approach to GW propagation; we extend it for accounting the possibility of having rapidly moving scalar fluctuations, with non minimal couplings to the metric. Within this framework, in Sections 2 and 3 we derive general formulas for describing the propagation of GWs, whose structure does not depend on the specific choice of the scalar-tensor theory under consideration, nor on the particular background over which the high-frequency GW is travelling. We only need to make (physically well founded) assumptions on the structure of the EMT controlling the propagation of high-frequency fluctuations ${ }^{1}$, and then apply our arguments within a geometrical optics approximation. Besides well-known modified gravity consequences for the evolution of the amplitude of tensor fluctuations - related with non-conservation of the effective Planck mass along the null GW geodesics - we also point out potentially interesting effects associated with the failure of parallel transport the polarization tensors along the GW geodesics. Although these latter effects can be vanishingly small for GWs propagating through smooth, homogeneous space-times, they can instead contribute to the evolution of the polarization tensors through the inhomogeneous Universe. GW evolution equations within GR, which include effects of non-parallel transport for the polarization tensors have been studied in a framework beyond geometrical optics, see e.g. [11, 50-53], associating it with (higher order) GW lensing effects (see e.g. [54-56]). Interestingly, we find that these effects can be present also within a geometrical optics approximation in theories of modified gravity. Over the past decades, various classes scalar-tensor theories have been applied to cosmology: Brans-Dicke [57]; K-essence [58]; Galileons [59], Horndeski [60-62],

\footnotetext{
${ }^{1}$ We focus on set-up with luminal speed for the spin-2 modes, as suggested by the GW170817 observations.
} 
beyond-Horndeski [63, 64], and DHOST [65-71]. These are increasingly complex theories with specific derivative couplings between scalar and tensor degrees of freedom. Our general approach can be in principle applied to any of these theories (and possibly beyond, if one admits Lorentz-violating scenarios) although for this work we focus only on scenarios in which the scalar mode propagates at the same speed of tensor degrees of freedom. In Section 4 we apply our approach to a specific case of the kinetic-gravity braiding set-up developed in [72], analyzing the differences with respect to GR for what concerns the evolution of GWs. Finally, in Section 5 we apply these formulas to GW propagation through a perturbed spacetime, to understand the role of modified gravity effects for propagation in an inhomogeneous universe.

In fact, we wish to close our Introduction with more detailed arguments for motivating our study. The physics of GWs travelling through cosmological distances can provide valuable information on gravitational interactions and on the properties of space-time between the emission and detection of GWs. In the literature, the effect of cosmological perturbations on the propagation of GWs has been often neglected. On the other hand, we find it timely to investigate these subtle effects to understand possible contaminations and degeneracies, so to obtain a more reliable estimates of cosmography. Obviously a similar analysis, for the correlated fluctuations in luminosity distance of the electromagnetic spectrum, has already and widely been discussed in literature, e.g. see [73-77]. Recently there have been several initial attempts to investigate the Integrated-Sachs Wolfe (ISW) effect on GWs from supermassive black hole mergers and in particular its impact on the system's parameter estimation [78], the ISW of a primordial stochastic background [79-81] and for astrophysical stochastic background in [82-93]. In these works the authors consider the presence of inhomogeneities in the matter distribution and allow to probe GW's sources on cosmological, galactic and sub-galactic scales, peculiar velocity [94-96] and lensing effects [27, 97-103]. [104] examines the GW memory, a permanent displacement of the gravitational wave detector after the wave has passed, in a highly inhomogeneous universe. It is worth noticing that environmental effects can also influence estimates of the luminosity distance [105]. Precisely, these sources are affected by coherent peculiar velocity of the merging at low redshift and weak gravitational lensing by intervening inhomogeneities in the cosmic mass distribution. Both these systematic errors could introduce a misidentification of the host's redshift. Consequently, changes of typically a few percent (but occasionally much larger) in the flux are introduced which do not significantly affect the redshift, providing a source of noise in the luminosity distance redshift relation [35, 97]. Very recently, in [107], the authors discuss the effect of cosmological perturbations and inhomogeneities on estimates of the luminosity distance of black hole (BH) or neutron star (NS) binary mergers through gravitational waves. They applied the "Cosmic Rulers" formalism [108] and considered the observer frame as reference system and they derived a different expression wrt [78], which is correct for the effect of large-scale structures on GW waveforms, accounting for lensing, Sachs-Wolfe, integrated Sachs-Wolfe, time delay and volume distortion effects, and evaluate their importance for future GW experiments. [107] showed that the amplitude of the corrections is important and cannot be negligible for future interferometers as the ET, DECIGO and Big Bang Observer (BBO). From [107], the additional luminosity distance uncertainty, arising because of the inclusion of perturbations, has a peak at low- $z$ due to velocity contributions that surpasses the predicted measurement errors for all the experiments considered here. However, peculiar velocity effects rapidly decrease as $z$ increases. On the other hand lensing, which is an integrated effect, increases with the redshift of the source $z$, and its amplitude is of a factor $\sim 10$ smaller than ET forecast pre- 
cision ( $\sim 2$ for DECIGO). For BBO, the correction to the luminosity distance is consistently twice the predicted errors, making it a very relevant correction, that one will need to take into account. Making use of the weak-lensing magnification effect on a GW from a compact binary object, in [32] is showed that it is possible to discriminate the concordance $\Lambda$ CDM cosmological model and up-to-date competing alternatives as dynamical dark energy models (DE) or modified gravity theories (MG) parametrized with the usual two free phenomenological functions that modify the perturbed Einstein equations relating the matter density contrast to the lensing and the Newtonian potential (e.g. see [109]). Finally, for the effect of lensing magnification, in [97], the authors pointed out that for the uncertainty in the distance to an ensemble of GW's sources is not completely correct to use the standard deviation of the lensing magnification distribution divided by the square root of the number of sources. They showed that by exploiting the non-Gaussian nature of the lensing magnification distribution, it is possible to improve this distance determination, typically by a factor of 2 to 3 .

\section{General formalism}

In this Section we develop a general formalism aimed to describe the propagation of highfrequency gravitational waves (GWs), in the framework of scalar-tensor theories.

While the evolution of spin- 2 and spin-0 degrees of freedom decouple around conformally flat space-times (as for example homogeneous FRW universes), more care is needed when describing GW propagation around perturbed backgrounds - above all in scalar-theories with kinetic mixing between different fields, motivated by recent approaches to the dark energy problem. To address this topic in general terms, in this Section we propose an approach based on an expansion of the evolution equations in an high-frequency parameter $\epsilon$. Such approach makes use of a convenient unitary gauge to deal with scalar fluctuations. It does not require us to specify the exact structure of the scalar-tensor theory we consider but only at which order in the small parameter $\epsilon$ it contributes to the energy momentum tensor controlling the high-frequency fluctuations. The general set of evolution equations we obtain in this Section shall be then applied in Section 3 to a specific geometrical optics Ansatz.

\subsection{Linearized equations of motion: high and low frequency modes}

Geometrical optics for gravitational waves (GWs) is a well developed subject, which started with the systematic analysis of Isaacson [48, 49], and that is now part of well-known textbooks discussing GW physics (see e.g. [110]). It corresponds to the limit where GWs are characterized by a short wavelength, while the background space-time, over which the wave propagates, varies over much larger scales. Such distinction between two characteristic length scales allows one to define a hierarchy and a 'small' parameter used for a derivative expansion. Most works consider geometrical optics for scalar-tensor systems in which the scalar is minimally coupled with gravity (see e.g. the book [111] and references therein). On the other hand, for analyzing more modern scalar-tensor theories aimed to explain dark energy, the standard formalism is generally not sufficient, since it does not satisfactorily catch possible effects associated with derivative self-interactions and with non-minimal couplings of scalar to tensor degrees of freedom. The latter scenarios are well motivated by theories of dark energy exhibiting self-accelerating solutions (see e.g. [6] for a review). Our purpose in this Section is to provide an extension able to eventually include also these models (and possibly additional ones), trying to be as general and flexible as possible. 
In the system we consider, the metric $g_{\mu \nu}$ satisfies the gravitational field equations

$$
R_{\mu \nu}=\frac{8 \pi G_{N}}{c^{4}}\left(T_{\mu \nu}-\frac{1}{2} g_{\mu \nu} T\right)
$$

where the left hand side (LHS) contains only geometrical quantities depending on the metric (the Ricci tensor), while the right hand side (RHS) contributions from the extra scalar coupled with the spin-2 excitations, and possibly additional matter fields. We concentrate all the effects of 'modified gravity' in the RHS of the equation.

We assume that the tensor $g_{\mu \nu}$ can be separated into a slowly varying background $\bar{g}_{\mu \nu}$, and high frequency small fluctuations $h_{\mu \nu}$, which represent the GWs propagating over the smooth space-time:

$$
g_{\mu \nu}=\bar{g}_{\mu \nu}+h_{\mu \nu}, \quad \text { with } \quad\left|h_{\mu \nu}\right| \ll\left|\bar{g}_{\mu \nu}\right| .
$$

The background metric is characterized by the length scale $L_{B}$, while the wavelength of the wave is $\lambda$, such that the derivatives of these quantities scale as

$$
\partial \bar{g} \sim \frac{1}{L_{B}}, \quad \partial h \sim \frac{1}{\lambda} \quad \text { and } \quad \lambda \ll L_{B} .
$$

In this context, the background metric $\bar{g}_{\mu \nu}$ is general as long as it satisfies Eq. (2.2) and Eq. (2.3). In particular, on cosmological scales it does not necessarily describe an isotropic and homogeneous Universe. We shall make use of this fact in Section 5.

Our system contains also a scalar field $\varphi$, whose value can be separated in a smooth contribution $\bar{\varphi}$, plus a rapidly varying fluctuation

$$
\varphi=\bar{\varphi}+\varphi_{r},
$$

analogously to the metric decomposition in Eq. (2.2). We assume that the typical wavelength of the scalar fluctuation $\varphi_{r}$ of order $\lambda$ and the variation length scale of $\bar{\varphi}$ of order $L_{B}$. As discussed in the Introduction, for the class of scalar-tensor theories we are interested in, the background scalar field acquires a non-null vacuum expectation value (vev) $\bar{\varphi}$, associated with the physics governing the late-universe cosmological acceleration. The smooth vev $\bar{\varphi}$ normally depends on coordinates, and its gradient allows us to define a vector

$$
\bar{v}^{\mu}=\bar{\nabla}^{\mu} \bar{\varphi},
$$

slowly varying over the background. For cosmological applications such vector is typically time-like, $\bar{v}^{\mu} \bar{v}_{\mu}<0$.

We plug the decomposition of the metric Eq. (2.2) in the field equations of Eq. (2.1). We organize the expansion of the Ricci tensor as

$$
R_{\mu \nu}=\bar{R}_{\mu \nu}+R_{\mu \nu}^{(1)}+R_{\mu \nu}^{(2)}+\mathcal{O}\left(h^{3}\right),
$$

where $R_{\mu \nu}^{(1)}$ and $R_{\mu \nu}^{(2)}$ denote respectively linear and second order terms in $h_{\mu \nu}$ and are of the order $\sim h / \lambda^{2}$ and $\sim h^{2} / \lambda^{2}$ respectively, while $\bar{R}_{\mu \nu}$ only depends on $\bar{g}_{\mu \nu}$. The RHS of Eq. (2.1) may depend on the metric and on the scalar and matter fields. We can apply the same reasoning as for the Ricci and expand it at second order in the metric fluctuations. In this way Eq. (2.1) becomes

$$
\bar{R}_{\mu \nu}+R_{\mu \nu}^{(1)}+R_{\mu \nu}^{(2)}=\frac{8 \pi G_{N}}{c^{4}}\left[\left(\bar{T}_{\mu \nu}-\frac{1}{2} \bar{g}_{\mu \nu} \bar{T}\right)+\left(T_{\mu \nu}-\frac{1}{2} g_{\mu \nu} T\right)^{(1)}+\left(T_{\mu \nu}-\frac{1}{2} g_{\mu \nu} T\right)^{(2)}\right] \text {. }
$$




\subsection{On the averaging procedure}

It is possible to separate between high and low-frequency modes by averaging Eq. (2.7) over length scales large compared to $\lambda$ and small compared to $L_{B}$, i.e. over volumes of size $\bar{l}$ with

$$
\lambda \ll \bar{l} \ll L_{B} .
$$

This procedure has been known in literature as ADM-averaging scheme and is well described in [48]. The effect of such averages is to extract the slowly varying part of a quantity as its contribution is almost constant throughout the volume of integration, while the rapidly oscillating one averages out to zero. We can find the equation of motion of the high-frequency part by subtracting from Eq. (2.7) the slow part extracted through the averaging. In particular, in Eq. (2.7) we have

i) Barred quantities that survive the averaging, e.g. $\left\langle\bar{R}_{\mu \nu}\right\rangle_{\bar{l}}=\bar{R}_{\mu \nu}$.

ii) Linear quantities in $h_{\mu \nu}$ that do not survive the averaging, e.g. $\left\langle R_{\mu \nu}^{(1)}\right\rangle_{\bar{l}}=0$.

iii) Quadratic quantities in $h_{\mu \nu}$ that may survive the averaging. For instance, in a quadratic contribution as $h_{\mu \nu} h_{\rho \sigma}$, a mode with a high frequency wave-vector $\mathbf{k}_{1}$ from $h_{\mu \nu}$ can combine with a mode with a high wave-vector $\mathbf{k}_{2} \approx-\mathbf{k}_{1}$ from $h_{\rho \sigma}$ to give a low frequency wave-vector mode.

Using these prescriptions, after taking the average of Eq. (2.7), we can split Eq. (2.7) into

$$
\begin{aligned}
& \bar{R}_{\mu \nu}=\left(\bar{T}_{\mu \nu}-\frac{1}{2} \bar{g}_{\mu \nu} \bar{T}\right)-\left\langle R_{\mu \nu}^{(2)}\right\rangle_{\bar{l}}+\left\langle\left(T_{\mu \nu}-\frac{1}{2} g_{\mu \nu} T\right)^{(2)}\right\rangle_{\bar{l}}, \\
& R_{\mu \nu}^{(1)}=\left(T_{\mu \nu}-\frac{1}{2} g_{\mu \nu} T\right)^{(1)}-\left[R_{\mu \nu}^{(2)}\right]^{h i g h}+\left(T_{\mu \nu}^{(2)}-\frac{1}{2}\left(g_{\mu \nu} T\right)^{(2)}\right)^{h i g h},
\end{aligned}
$$

where the over-script high means $\left[R_{\mu \nu}^{(n)}\right]^{h i g h}=R_{\mu \nu}^{(n)}-\left\langle R_{\mu \nu}^{(n)}\right\rangle_{\bar{l}}$.

\subsection{The derivative expansion}

In light of these arguments, we can define separate expansions for the quantities entering in the field equations:

i) An expansion in small fluctuations $h_{\mu \nu}$, and (independently)

ii) an expansion in derivatives of the high-frequency modes, controlled by the small parameter

$$
\epsilon \equiv \lambda / L_{B}
$$

The mutual relationship between the two parameters is set by Eq. (2.8). If the background curvature is dominated by the matter contribution, Eq. (2.8) sets

$$
\frac{1}{L_{B}^{2}}=\frac{h^{2}}{\lambda^{2}}+(\text { matter contribution })+(\text { dark energy contribution }) \gg \frac{h^{2}}{\lambda^{2}},
$$

meaning that the hierarchy between the amplitude of GW and ratio of GW/background characteristics wavelengths is [110]

$$
1 \gg \frac{\lambda}{L_{B}} \gg h \quad \text { thus } \quad \epsilon \gg h
$$


This is not always the case: for instance, there may be situations in which the background curvature is determined by the gravitational waves content. In this case the relationship between the two parameters would be $\epsilon=h$ [110]. However, one of our goals is to study the propagation of GWs through the cosmic inhomogeneities where the background spacetime is completely determined by the large-scale structures present in the Universe. In this case the hierarchy in Eq. (2.12) holds. The $\epsilon$-expansion is meant to single out high frequency contributions to the equations: each derivative of high-frequency fluctuations $\left(h_{\mu \nu}\right.$, $\varphi_{r}$ ) collects a factor $1 / \epsilon$, which allows one to clearly separate independent contributions to the evolution equations, which are controlled by powers of $\epsilon$.

The hierarchy Eq. (2.12) allows us to discard the quadratic terms in $h_{\mu \nu}$ of Eq. (2.8) and Eq. (2.9), and expand what is left in powers of $\epsilon$ because $\epsilon \gg h \epsilon \gg h \epsilon^{2} \gg \cdots \gg h^{2}$. We have the background metric field equation

$$
\bar{R}_{\mu \nu}=\frac{8 \pi G_{N}}{c^{4}}\left(\bar{T}_{\mu \nu}-\frac{1}{2} \bar{g}_{\mu \nu} \bar{T}\right),
$$

which is the fully non-linear Einstein equation solved by the slowly-varying metric $\bar{g}_{\mu \nu}$, turning off the high-frequency fluctuations. The linearized equations for the perturbations are

$$
\begin{aligned}
& {\left[R_{\mu \nu}^{(1)}\right]_{1 / \epsilon^{2}}=0,} \\
& {\left[R_{\mu \nu}^{(1)}\right]_{1 / \epsilon}=\frac{8 \pi G_{N}}{c^{4}}\left[\left(T_{\mu \nu}-\frac{1}{2} g_{\mu \nu} T\right)^{(1)}\right]_{1 / \epsilon},} \\
& {\left[R_{\mu \nu}^{(1)}\right]_{\epsilon^{0}}=\frac{8 \pi G_{N}}{c^{4}}\left[\left(T_{\mu \nu}-\frac{1}{2} g_{\mu \nu} T\right)^{(1)}\right]_{\epsilon^{0}},}
\end{aligned}
$$

the subscript $\epsilon^{p}$ means that the equality holds at that order in the expansion in power of $\epsilon$.

The limit of geometrical optics consists in focusing on the previous equations at leading and next-to-leading order in an $\epsilon$ expansion: that is, on Eq. (2.14) and Eq. (2.15). For sake of clearness, we write the RHS of the equations above explicitly

$$
\left(T_{\mu \nu}-\frac{1}{2} g_{\mu \nu} T\right)^{(1)}=T_{\mu \nu}^{(1)}-\frac{1}{2} \bar{g}_{\mu \nu} T^{(1)}-\frac{1}{2} h_{\mu \nu} \bar{T}+\frac{1}{2} \bar{g}_{\mu \nu} h^{\alpha \beta} \bar{T}_{\alpha \beta}
$$

where we defined

- $\bar{T}_{\mu \nu}$ is the background stress-energy tensor built with $\bar{g}_{\mu \nu}$ and $\bar{T}=\bar{g}^{\mu \nu} \bar{T}_{\mu \nu}$,

- $T_{\mu \nu}^{(1)}$ is the linear in $h_{\mu \nu}$ stress-energy tensor and $T^{(1)}=\bar{g}^{\mu \nu} T_{\mu \nu}^{(1)}$.

The last two terms of Eq. (2.17) do not contain derivatives of the metric perturbation $h_{\mu \nu}$, so they will not contribute to Eq. (2.15) but only to Eq. (2.16), namely

$$
\left[T_{\mu \nu}^{(1)}-\frac{1}{2} \bar{g}_{\mu \nu} T^{(1)}-\frac{1}{2} h_{\mu \nu} \bar{T}+\frac{1}{2} \bar{g}_{\mu \nu} h^{\alpha \beta} \bar{T}_{\alpha \beta}\right]_{1 / \epsilon^{1}}=\left[T_{\mu \nu}^{(1)}-\frac{1}{2} \bar{g}_{\mu \nu} T^{(1)}\right]_{1 / \epsilon^{1}},
$$

therefore we can rewrite Eq. (2.15) as

$$
\left[R_{\mu \nu}^{(1)}\right]_{1 / \epsilon^{1}}=\frac{8 \pi G_{N}}{c^{4}}\left[T_{\mu \nu}^{(1)}-\frac{1}{2} \bar{g}_{\mu \nu} T^{(1)}\right]_{1 / \epsilon^{1}} .
$$




\subsection{On the structure of the energy-momentum tensor $T_{\mu \nu}$}

Usually, when describing propagation of GWs over cosmological distances, it is assumed that the energy-momentum tensor (EMT) $T_{\mu \nu}$ experienced by GWs is smooth, and does not affect the high-frequency GW evolution in the limit of geometrical optics (see e.g. [110]). Under such hypothesis, both the RHS of Eq. (2.14) and of Eq. (2.19) vanish, and the EMT does not contribute to the GW evolution in the geometrical optics limit. But this hypothesis can be too restrictive in scalar-tensor theories, where derivative scalar self-interactions can lead to derivative contributions to the EMT involving high-frequency modes. As an example, the EMT associated with a cubic Galileon model described by the Lagrangian density

$$
\mathcal{L}=-\frac{1}{2}\left(\partial_{\mu} \varphi \partial^{\mu} \varphi\right) \square \varphi
$$

contains second covariant derivatives of the scalar, $\nabla_{\mu} \nabla_{\nu} \varphi$ that - through the Christoffel symbols contained in the $\nabla_{\mu} \nabla_{\nu}$ operator - lead to first derivatives of the high-frequency metric fluctuations. (We will study in more detail this and other models in Section 4.)

In this case, as well as in other modified gravity scenarios, one then expects contributions to the EMT at next-to-leading order in an $\epsilon$-expansion, affecting a geometrical optics description. For the rest of this Section and in Section 3, therefore, we assume to have a non-vanishing contribution to the RHS of Eq. (2.19), associated with single derivatives of the high-frequency modes. Without further specifying the theory one considers, we can then derive general consequences of this hypothesis for what concerns GW propagation in the framework of geometrical optics ${ }^{2}$.

Eq. (2.16) at order $\mathcal{O}\left(\epsilon^{0}\right)$, as well as other equations characterized by higher powers of $\epsilon$, will not be considered, since they do not contribute in the limit of geometrical optics (where $\epsilon \ll 1$ ). When expanded at order $\mathcal{O}\left(\epsilon^{0}\right)$, Eq. (2.16) acquires extra contributions after performing a coordinate transformation, and it is not easily related to a physical observable. This fact is discussed in [48] for the case of GR. In next Section, we show that the same argument holds also in the more general scenario we study.

\subsection{Gauge choice and evolution equations}

We now discuss how to choose a convenient gauge choice for high frequency fluctuations, and the resulting equations of motion for the propagating modes. We shall adopt a unitary gauge that sets to zero high frequency fluctuations associated with the scalar $\varphi_{r}$. This choice turns out to be convenient for physically motivating our Ansatz, and the applications we develop in Section 3. Note that, within this gauge that sets to zero the rapidly-varying scalar fluctuations, the dark energy scalar field can still be characterized by slowly varying dynamical perturbations.

\subsubsection{A convenient gauge for theories with a single extra high-frequency mode}

We make the hypothesis that the scalar-tensor system one considers transforms in the standard way under diffeomorphism transformations. As discussed in $[48,110]$, gauge transformations can be organized in inverse powers of an expansion in $\epsilon$. Calling $\xi^{\mu}$ the high-frequency

\footnotetext{
${ }^{2}$ For simplicity we shall only allow for contributions to the EMT with a single derivative acting on high frequency fields, i.e. at order $1 / \epsilon$. As it will become clear in section 3 , the presence of two or higher derivatives in such fields could affect our hypothesis that gravitons propagate on null geodesics, and at the speed of light.
} 
part of the the coordinate transformation $x^{\mu} \rightarrow x^{\mu}+\xi^{\mu}$, the high frequency GW metric fluctuations and high frequency scalar field fluctuation transform in curved space as

$$
\begin{aligned}
& h_{\mu \nu} \rightarrow h_{\mu \nu}-\left(\bar{\nabla}_{\mu} \xi_{\nu}+\bar{\nabla}_{\nu} \xi_{\mu}\right), \\
& \varphi_{r} \rightarrow \varphi_{r}+\bar{v}^{\rho} \xi_{\rho},
\end{aligned}
$$

where the bars on $\bar{\nabla}_{\mu}$ mean covariant derivatives with respect to the background curved metric $\bar{g}_{\mu \nu}$ and $\bar{v}^{\mu}=\bar{\nabla}^{\mu} \bar{\varphi}$ as defined in Eq. (2.5). From now on, space-time indexes are always raised and lowered with the background metric $\bar{g}_{\mu \nu}$.

As the decompositions Eq. (2.2) and Eq. (2.4) have to hold also in the new coordinate system, we require that the vector field $\xi_{\mu}$ satisfy the following inequalities

$$
\left|\xi_{\nu}\right| \lesssim h L_{B} \quad \text { and } \quad\left|\partial_{\mu} \xi_{\nu}\right| \lesssim h \quad \text { and } \quad\left|\xi_{\nu}\right| \lesssim \varphi_{r} L_{B}
$$

in this way the background is left unchanged when performing a gauge transformation. These requirements together with Eq. (2.22) imply that

$$
\varphi_{r} \sim h \epsilon,
$$

so, when considering an expansion in the quantity $\epsilon$, the amplitude of the rapidly varying scalar fluctuation is one order of magnitude smaller than the amplitude of the metric fluctuations.

The high-frequency contributions to the linearized Ricci tensor and of the EMT are gauge invariant up to next-to leading order in the $1 / \epsilon$ expansion $[48,110]$. Indeed, under a gauge transformation, they transform as (we focus on the Ricci tensor as an example)

$$
R_{\mu \nu}^{(1)} \rightarrow R_{\mu \nu}^{(1)}=R_{\mu \nu}^{(1)}-\mathcal{L}_{\xi} \bar{R}_{\mu \nu},
$$

where $\bar{R}_{\mu \nu}$ refers to the quantity evaluated with the background metric, and $\mathcal{L}_{\xi}$ is the Lie derivative along the vector field $\xi$. In general the Lie derivatives will be different from zero, in fact $\mathcal{L}_{\xi} \bar{R}_{\mu \nu} \sim \epsilon^{0}$, therefore $R_{\mu \nu}^{(1)} \rightarrow R_{\mu \nu}^{(1)}$ only at $1 / \epsilon^{2}$ and $1 / \epsilon$ orders. This means that Eq. (2.14) and Eq. (2.19) are gauge invariant, while Eq. (2.16) is not. The reason behind this fact is that, on a scale of distance of order $\lambda$, the space-time appears locally flat and the curvature is locally gauge invariant. As long as $\lambda \ll L_{B}$, perturbations do not have any long-wavelength component and this local behavior carries over to curved background, to give a global gauge invariance [48] which is a result of our high-frequency assumptions. Since we will never use quantities at $\mathcal{O}\left(\epsilon^{0}\right)$, we adopt the following prescription in our computation: we keep only terms at $1 / \epsilon^{2}$ and $1 / \epsilon$ orders, and neglect those at $\epsilon^{0}$ onward.

It is convenient to work with the trace-reversed metric perturbation defined as

$$
\hat{h}_{\mu \nu}=h_{\mu \nu}-\frac{1}{2} \bar{g}_{\mu \nu} h,
$$

with $h_{\mu}^{\mu}=h$.

We are interested in gauge-fixing $\hat{h}_{\mu \nu}$ so that it is transverse, and set to zero the highfrequency scalar field oscillation $\varphi_{r}=0$. So we focus on the transformation laws (the primes indicate quantities after acting with a gauge transformation)

$$
\begin{aligned}
& \varphi_{r}^{\prime}=\varphi_{r}+\bar{v}^{\rho} \xi_{\rho}, \\
& \bar{\nabla}^{\nu} \hat{h}_{\mu \nu}^{\prime}=\bar{\nabla}^{\nu} \hat{h}_{\mu \nu}+\bar{\nabla}^{\rho} \bar{\nabla}_{\rho} \xi_{\mu}-\bar{R}_{\mu \nu} \xi^{\nu},
\end{aligned}
$$


and choose $\xi^{\mu}$ such that

$$
\bar{\nabla}^{\rho} \bar{\nabla}_{\rho} \xi_{\mu}=-\bar{\nabla}^{\nu} \hat{h}_{\mu \nu},
$$

where we neglect the contribution proportional to $\bar{R}_{\mu \nu}$ in the transformation law of $\bar{\nabla}^{\mu} \hat{h}_{\mu \nu}$, since it is lower at order $\mathcal{O}\left(\epsilon^{0}\right)$ in an $\epsilon$-expansion. We then find

$$
\bar{\nabla}^{\nu} \hat{h}_{\mu \nu}^{\prime}=0, \quad \text { and } \quad \varphi_{r}^{\prime}=\varphi_{r}+\bar{v}^{\rho} \xi_{\rho} .
$$

We can make the further transformation $x^{\mu} \rightarrow x^{\mu}+\chi^{\mu}$ with $\square \chi^{\mu}=0$ that does not spoil the harmonic gauge,

$$
\varphi_{r}^{\prime \prime}=\varphi_{r}+\bar{v}^{\rho}\left(\xi_{\rho}+\chi_{\rho}\right), \quad \text { and } \quad \bar{\nabla}^{\nu} \hat{h}_{\mu \nu}^{\prime \prime}=0,
$$

which allows us to set $\varphi_{r}^{\prime \prime}=0$ by taking

$$
v^{\mu} \chi_{\mu}=-\left(\varphi_{r}+\bar{v}^{\rho} \xi_{\rho}\right)
$$

so that we are finally left with

$$
\varphi_{r}^{\prime \prime}=0, \quad \text { and } \quad \bar{\nabla}^{\nu} \hat{h}_{\mu \nu}^{\prime \prime}=0,
$$

as desired.

We point out that there is another residual gauge freedom given by $x^{\mu} \rightarrow x^{\mu}+\sigma^{\mu}$ with $\bar{\square} \sigma^{\mu}=\bar{v}^{\mu} \sigma_{\mu}=0$ that leaves Eq. (2.33) unchanged. We opt to make use of this remaining freedom to fix the quantity

$$
c_{\mu} \equiv \bar{v}^{\nu} \hat{h}_{\mu \nu}
$$

that will play an important role in our discussion, and whose transformation law follows from the one of $\hat{h}_{\mu \nu}$. Indeed, under $x^{\mu} \rightarrow x^{\mu}+\left(\xi^{\mu}+\chi^{\mu}+\sigma^{\mu}\right)$ the vector $c_{\mu}$ transforms as

$$
\begin{aligned}
c_{\mu}^{\prime \prime \prime} & =c_{\mu}-\bar{v}^{\nu}\left(\bar{\nabla}_{\nu}\left(\xi_{\mu}+\chi_{\mu}+\sigma_{\mu}\right)+\bar{\nabla}_{\mu}\left(\xi_{\nu}+\chi_{\nu}+\sigma_{\nu}\right)-\bar{g}_{\mu \nu} \bar{\nabla}^{\rho}\left(\xi_{\rho}+\chi_{\rho}+\sigma_{\rho}\right)\right) \\
& =c_{\mu}^{\prime \prime}-\bar{v}^{\nu}\left(\bar{\nabla}_{\nu} \sigma_{\mu}+\bar{\nabla}_{\mu} \sigma_{\nu}-\bar{g}_{\mu \nu} \bar{\nabla}^{\rho} \sigma_{\rho}\right)
\end{aligned}
$$

with $c_{\mu}^{\prime \prime}=\bar{v}^{\nu} \hat{h}_{\mu \nu}^{\prime \prime}$. From the transversality of $\hat{h}_{\mu \nu}^{\prime}$ it follows that $c_{\mu}^{\prime}$ is divergenceless at leading and next-to-leading order, in fact

$$
\bar{\nabla}^{\mu} c_{\mu}^{\prime}=\bar{\nabla}^{\mu}\left(\bar{v}^{\nu} \hat{h}_{\mu \nu}^{\prime}\right)=\bar{v}^{\nu} \bar{\nabla}^{\mu} \hat{h}_{\mu \nu}^{\prime}=0
$$

and the same holds for $c_{\mu}^{\prime \prime}$ and $c_{\mu}^{\prime \prime \prime}$. This can be checked also by taking the divergence of Eq. (2.35). However, from Eq. (2.29) we see that $\bar{\nabla}^{\mu} c_{\mu}=-\bar{v}^{\mu} \square \xi_{\mu}$. Hence, from Eq. (2.35) we learn that using the residual gauge condition associated with the vector $\sigma_{\mu}$, we can make our desired choice for the vector $c_{\mu}$, fixing the last remaining gauge freedom. Notice that whatever our initial choice for $c_{\mu}$ is, this vector shall have to satisfy conditions of compatibility with the metric equations of motion, implying that in general its value will not be covariantly preserved over the GW null geodesics. We shall concretely discuss in Section 3 how the $c_{\mu^{-}}$ evolution is controlled by the equations of motion for the high-frequency metric fields. In the case of null waves, both scalar and tensor, one can choose to use the last gauge freedom associated to $\sigma_{\mu}$ to fix the scalar longitudinal and vector modes present in the wave content. In Appendix B.1 we show how it is possible to do so. 


\subsubsection{Compatibility among our gauge choices}

We have learned that it is in principle possible to select at the same time a unitary gauge for the rapid scalar fluctuations, and a transverse gauge for the rapid metric fluctuations, as in Eq. (2.33). We now examine whether there are requirements to impose on the system for ensuring the compatibility of these two gauge conditions. As we have seen, the possibility of choosing the gauge Eq. (2.33) is guaranteed if there exist a vector $\chi^{\mu}$ such that

$$
\begin{aligned}
& \square \chi_{\mu}=0, \\
& \bar{v}^{\rho} \chi_{\rho}=-\left(\varphi_{r}+\bar{v}^{\rho} \xi_{\rho}\right) .
\end{aligned}
$$

In order to understand weather this is allowed, we take $\bar{\square}$ on both the left and right hand sides of Eq. (2.38), and after some manipulations, we arrive to

$$
\bar{\square} \varphi_{r}-\bar{\nabla}^{\mu} c_{\mu}=-\left(\chi_{\rho}+\xi_{\rho}\right) \bar{\square} \bar{v}^{\rho}-2 \bar{\nabla}^{\mu} \bar{v}^{\rho} \bar{\nabla}_{\mu}\left(\chi_{\rho}+\xi_{\rho}\right) .
$$

The orders of magnitude of the vector fields on the RHS of the previous equation is restricted by Eq. (2.23) so that

$$
\begin{aligned}
& -\left(\chi_{\rho}+\xi_{\rho}\right) \bar{\square} \bar{v}^{\rho} \lesssim h L_{B}^{-2} \\
& -2 \bar{\nabla}^{\mu} \bar{v}^{\rho} \bar{\nabla}_{\mu}\left(\chi_{\rho}+\xi_{\rho}\right) \lesssim h L_{B}^{-2}
\end{aligned}
$$

so the RHS of Eq. (2.39) does not have contributions at leading $\left(\epsilon^{-2}\right)$ and next to leading $\left(\epsilon^{-1}\right)$ and therefore the compatibility condition is given by

$$
\left[\bar{\square} \varphi_{r}-\bar{\nabla}^{\mu} c_{\mu}\right]_{1 / \epsilon^{2}, 1 / \epsilon^{1}}=0 .
$$

The compatibility condition Eq. (2.42) can be also stated in a more physically transparent way in terms of a linearized equation for the scalar fluctuation

$$
\left[(\square \varphi)^{(1)}\right]_{1 / \epsilon^{2}, 1 / \epsilon^{1}}=0,
$$

because, up to sub-leading contributions that we neglect, we have

$$
\begin{aligned}
(\square \varphi)^{(1)}=\bar{g}^{\mu \nu}\left(\varphi_{\mu \nu}\right)^{(1)} & =\bar{g}^{\mu \nu}\left(\bar{\nabla}_{\mu} \bar{\nabla}_{\nu} \varphi_{r}-\frac{\bar{\varphi}^{\sigma}}{2}\left(\bar{\nabla}_{\mu} h_{\sigma \nu}+\bar{\nabla}_{\nu} h_{\sigma \mu}-\bar{\nabla}_{\sigma} h_{\mu \nu}\right)\right)= \\
& =\square \varphi_{r}-v^{\sigma} \bar{\nabla}^{\mu} \hat{h}_{\sigma \mu}=\square \varphi_{r}-\bar{\nabla}^{\mu} c_{\mu} .
\end{aligned}
$$

We conclude that it is possible to choose simultaneously the gauge choices in Eq. (2.33) only if Eq. (2.42) - or equivalently Eq. (2.43) - is satisfied. This condition amounts to impose that rapidly moving linearized scalar fluctuations propagate freely - and at light speed - around the cosmological background under consideration. This fact typically imposes constraints on the scalar-tensor theories we can consider with our formalism, and on their background solutions. In Appendix A we discuss in the concrete setting of a general scalartensor system such constraint conditions. For the rest of our work, we then assume that the scalar-tensor systems and background configurations around which our rapidly moving fluctuations propagate are such that Eq. (2.43) is satisfied. 


\subsubsection{Summary of the gauge conditions and of the relevant equations of motion}

To briefly summarize, our system satisfies a unitary gauge for the high-frequency perturbations of the scalar field

$$
\varphi_{r}=0
$$

and a transverse (but not traceless) gauge for the fast moving part of the metric fluctuations:

$$
\bar{\nabla}^{\mu} \hat{h}_{\mu \nu}=0
$$

As a final condition, that exhausts our gauge freedom, we impose

$$
\bar{v}^{\mu} \hat{h}_{\mu \nu}=c_{\nu} \quad \text { with } \quad \bar{\nabla}^{\mu} c_{\mu}=0,
$$

where the initial value of $c_{\mu}$ can be chosen arbitrarily and its evolution along GW geodesics will be controlled by the condition of compatibility with the equations of motion for the high-frequency metric fields.

The number of propagating degrees of freedom (dofs) can be counted as follows. We start from 11 dofs: 1 in the scalar, 10 in the symmetric tensor $h_{\mu \nu}$. We have 1 gauge condition Eq. (2.45), 4 gauge conditions Eq. (2.46), 3 independent gauge conditions Eq. (2.47) $\left(c_{\nu}\right.$ is divergenceless). In total, we generically have $11-1-4-3=3$ independent propagating dofs, as expected in a scalar-tensor theory of gravity.

We shall use this gauge fixing and rewrite the relevant equations of motion Eqs. (2.14)(2.19), they become ${ }^{3}$

$$
\begin{aligned}
& {\left[\square \hat{h}_{\mu \nu}\right]_{1 / \epsilon^{2}}=0,} \\
& {\left[\square \hat{h}_{\mu \nu}\right]_{1 / \epsilon^{1}}=-\frac{16 \pi G_{N}}{c^{4}}\left[T_{\mu \nu}^{(1)}\right]_{1 / \epsilon^{1}} .}
\end{aligned}
$$

These are the evolution equations for the high frequency modes in our setting. Given that the only high frequency fields are the metric fluctuations $\hat{h}_{\mu \nu}$, the EMT appearing in Eq. (2.50) is built in terms of single derivatives of these quantities, that select the $1 / \epsilon$ contributions to the equations. (The concrete structure of $T_{\mu \nu}^{(1)}$ depends of course on the scalar-tensor theory one considers.)

Since we work in an unitary gauge for the high frequency scalar fluctuations, the metric fluctuations $\hat{h}_{\mu \nu}$ contain the extra degree of freedom which we expect to generically propagate in our system. Consequently, it does not correspond to a pure spin-2, transverse-traceless fluctuations of GR.

The compatibility of equations of motion and gauge conditions leads to an additional relation. We apply a covariant derivative $\bar{\nabla}^{\mu}$ on both sides of Eq. (2.50), and we select the $1 / \epsilon^{2}$ contributions. In the LHS we can interchange the order of covariant derivatives (the error being of order $1 / \epsilon$ hence negligible) and find $\square \bar{\nabla}^{\mu} \hat{h}_{\mu \nu}=0$ for the transverse gauge

\footnotetext{
${ }^{3}$ The linearized Ricci tensor, neglecting its contribution at $\epsilon^{0}$ order, in this gauge reads

$$
R_{\mu \nu}^{(1)}=-\frac{1}{2}\left(\bar{\square} \hat{h}_{\mu \nu}-\frac{1}{2} \bar{g}_{\mu \nu} \bar{\square} \hat{h}\right) .
$$
}


condition Eq. (2.46). In the RHS, we obtain a condition corresponding to the conservation of the energy momentum tensor:

$$
\left[\bar{\nabla}^{\mu} T_{\mu \nu}^{(1)}\right]_{1 / \epsilon^{2}}=0
$$

As typical in scalar-tensor systems, we expect such condition to be equivalent to the conditions provided by the high-frequency contributions to the scalar field equation in unitary gauge. Therefore, the three dynamical equations, at separate orders in $\epsilon$ expansion, are

$$
\begin{aligned}
{\left[\bar{\nabla}^{\rho} \bar{\nabla}_{\rho} \hat{h}_{\mu \nu}\right]_{1 / \epsilon^{2}} } & =0 \\
{\left[\bar{\nabla}^{\rho} \bar{\nabla}_{\rho} \hat{h}_{\mu \nu}\right]_{1 / \epsilon} } & =-\frac{16 \pi G_{N}}{c^{4}}\left[T_{\mu \nu}^{(1)}\right]_{1 / \epsilon} \\
{\left[\bar{\nabla}^{\mu} T_{\mu \nu}^{(1)}\right]_{1 / \epsilon^{2}} } & =0 .
\end{aligned}
$$

We now analyse all these equations in the framework of geometrical optics. Our considerations will be sufficiently general to be applied to any scalar-tensor system with a highfrequency contribution to its EMT, as described above.

\section{Geometrical optics with a single extra degree of freedom}

The analysis of Section 2 sets the stage to consistently treat the evolution of high frequency fluctuations in scalar-tensor settings, without specific hypothesis on the scalar-tensor theory and the background one considers. The effects of 'modified gravity' in the high-frequency field dynamics are enclosed in contributions to the EMT, built in terms of high-frequency fluctuations ${ }^{4}$. The scope of this Section is to apply this formalism to a framework of geometrical optics. We adopt a specific Ansatz that allows us to follow the evolution of the GW amplitude, phase, and GW polarization tensor, as a function of certain combinations of the EMT. We determine modified evolution equations for these quantities with respect to GR, and discuss the potential physical consequences.

We find that, for scalar-tensor theories of the kind we are interested in, GWs follow null geodesics, but the evolution equation for the GW amplitude is modified with respect to GR, leading to the phenomenon of non-conservation of the effective Planck mass. This is a well known property found in various works that study GW propagation in FRW space-times: our results generalize those findings to more general settings.

Interestingly, we also find that the polarization tensor is generally not parallel transported along the GW null geodesics. Possible implications of this fact for the evolution of GWs will then be discussed analyzing specific examples in the next Sections.

\subsection{Ansatz and evolution equations}

We start defining the geometrical optic Ansatz we adopt. The metric fluctuations can be expressed in terms of an amplitude and a phase as [48]

$$
\hat{h}_{\mu \nu} \equiv\left[\mathcal{A}_{\mu \nu}+\epsilon \cdots+\ldots\right] e^{i \theta / \epsilon}
$$

\footnotetext{
${ }^{4}$ In fact, we made the choice to include all the effects of modified gravity on the RHS of Einstein equations, while on the LHS we keep the traditional Einstein tensor.
} 
where all the parts with dots are higher order in the $\epsilon$ expansion described in Section 2, and will be neglected in the geometrical optics approximations we adopted here. The tensor $\mathcal{A}_{\mu \nu}$ contains both spin- 2 and spin- 0 contributions. Since they are part of the same metric tensor $\hat{h}_{\mu \nu}$ in the unitary gauge we adopt, we make the hypothesis that they have the same phase, hence they follow the same geodesics. This hypothesis is backed up by the fact that in our approach we restrict ourselves only to systems in which scalar waves and gravitational waves travel at the same speed. (In Section 3.3 we also investigate at what extent, within this hypothesis, the evolution of tensor and scalar modes can be studied independently.)

The tensorial quantity $\mathcal{A}_{\mu \nu}$ is decomposed into an amplitude $\mathcal{A}$ and a tensor polarization $\mathbf{e}_{\mu \nu}$ with unit norm

$$
\mathcal{A}_{\mu \nu} \equiv \mathcal{A} \mathbf{e}_{\mu \nu}
$$

with

$$
\mathcal{A}=\sqrt{\mathcal{A}_{\mu \nu} \mathcal{A}^{\mu \nu}}, \quad \mathbf{e}_{\mu \nu}=\frac{\mathcal{A}_{\mu \nu}}{\mathcal{A}}, \quad \mathbf{e}_{\mu \nu} \mathbf{e}^{\mu \nu}=1 .
$$

In the unitary gauge adopted, the high frequency contribution to the EMT is built with derivatives of the metric fluctuations, therefore we can assume it has the same phase as the metric fluctuation (we will give an explicit example in Section 4). We then adopt the following Ansatz for the EMT at order $1 / \epsilon$ (the overall coefficient is chosen such to simplify the following equations):

$$
\left[T_{\mu \nu}^{(1)}\right]_{1 / \epsilon} \equiv \frac{i}{\epsilon} \frac{c^{4}}{16 \pi G_{N}} t_{\mu \nu} e^{i \theta / \epsilon} .
$$

As explained in the previous Sections, the contributions that scale as $1 / \epsilon$ collect the terms proportional to first derivatives of the high-frequency fields. The tensor $t_{\mu \nu}$, consequently, is proportional to the coefficients of these terms. Importantly, notice that we collect in the coefficient of the RHS of Eq. (3.4) the quantity $G_{N}=\bar{M}_{\mathrm{Pl}}^{-2}$ with $\bar{M}_{\mathrm{Pl}}$ a reference, constant mass scale. The effective Planck scale experienced by the GWs can on the other hand vary along the GW trajectory - a phenomenon found in various examples of scalar-tensor theories. We make the hypothesis that the tensor $t_{\mu \nu}$ includes contributions containing this possibility, if realized in the scalar-tensor theories under consideration.

So far, we learned that the unitary gauge condition we adopted allow us to make the reasonable assumption that all the quantities we are dealing with in geometrical optics share the same phase. We will focus on this simplifying assumption throughout all this work. We also write

$$
\bar{v}^{\mu} \hat{h}_{\mu \nu}=c_{\nu}=\left[\mathcal{C}_{\nu}+\epsilon \ldots\right] e^{i \theta / \epsilon} .
$$

Finally, the wave-vector of the propagating GW is defined $\operatorname{as}^{5}$

$$
k_{\mu} \equiv-\partial_{\mu} \theta,
$$

and identifies the GW geodesics as we now show. The gauge conditions Eqs. (2.46)-(2.47), at leading and next-to-leading order, are given by

$$
\begin{aligned}
k^{\mu} \mathbf{e}_{\mu \nu} & =0, \\
\bar{v}^{\mu} \mathcal{A}_{\mu \nu} & =\mathcal{C}_{\nu}, \\
k^{\mu} \mathcal{C}_{\mu} & =0,
\end{aligned}
$$

\footnotetext{
${ }^{5}$ The minus sign in the definition of $k_{\mu}$ is because we use the comoving distance $\chi$ as affine parameter. The opposite convention is used in [78] because they parametrize the GW's geodesics with $\lambda=-\chi$.
} 
so that the polarization tensor $\mathbf{e}_{\mu \nu}$ and the vector $\mathcal{C}_{\mu}$ are transverse to the $\mathrm{GW}$ propagation. We can now plug the Anstaz (3.1) and (3.4) in the equations of motion Eqs. (2.52), (2.53) and (2.54).

From Eq. (2.52) we obtain

$$
k_{\mu} k_{\nu} \bar{g}^{\mu \nu}=0
$$

which means that the GWs wave-vector is a null vector of the slowly varying background $\bar{g}_{\mu \nu}$. Moreover, using the definition of $k_{\mu}$, one can show that it also satisfies

$$
k^{\mu} \bar{\nabla}_{\mu} k_{\nu}=0 .
$$

Thus, the GWs wave-vector is a geodesic vector. These two results allow us to conclude that, even in the more general case where $T_{\mu \nu}^{(1)}$ contains contributions at $1 / \epsilon$, GWs travel along null geodesics of the background manifold as in the case of GR with a smooth matter content. The hypothesis that $T_{\mu \nu}^{(1)}$ only contribute starting at order $1 / \epsilon$, and not $1 / \epsilon^{2}$, is crucial to ensure this property. Our assumption is motivated by the fact that the multi-messenger event GW170817 established that, in excellent approximation, GWs travel at the speed of light.

From Eq. (2.54) we obtain

$$
k^{\mu} t_{\mu \nu}=0,
$$

which states that the $1 / \epsilon$ order of $T_{\mu \nu}^{(1)}$ is transverse. This property is a direct consequence of the fact that $t_{\mu \nu}$ is built by the high-frequency metric fluctuations, which are transverse.

From Eq. (2.53) we obtain

$$
2 k_{\rho} \bar{\nabla}^{\rho}\left(\mathcal{A}_{\mu \nu}\right)+\mathcal{A}_{\mu \nu}\left(\bar{\nabla}^{\rho} k_{\rho}\right)=t_{\mu \nu},
$$

which can be separated into an equation for the amplitude $\mathcal{A}$

$$
\bar{\nabla}_{\rho}\left(k^{\rho} \mathcal{A}^{2}\right)=\mathcal{A} t_{\mu \nu} \mathbf{e}^{\mu \nu}
$$

and equation for the polarization $\mathbf{e}_{\mu \nu}$ :

$$
k_{\rho} \bar{\nabla}^{\rho} \mathbf{e}_{\mu \nu}=\frac{1}{2 \mathcal{A}}\left[t_{\mu \nu}-\mathbf{e}_{\mu \nu}\left(t_{\rho \sigma} \mathbf{e}^{\rho \sigma}\right)\right]
$$

by multiplying Eq. (3.13) by $\mathbf{e}^{\mu \nu}$ and using Eq. (3.3). Eqs. (3.14)-(3.15) control the evolution of the metric's fluctuations in the limit of geometrical optics. The difference with respect to standard geometrical optics in GR lies in their potentially non-vanishing right-hand-sides, controlled by the high-frequency contribution to the EMT $t_{\mu \nu}$, as defined in Eq. (3.4).

We propose the following interpretation of these results:

- Eq. (3.14) controls the evolution of the GW amplitude of the high-frequency GWs as they travel along a null geodesics. A non-vanishing RHS is associated with the non-conservation of the current $\mathcal{A}^{2} k^{\mu}$.

In scalar-tensor theories where the graviton number is conserved, the physical interpretation of this fact is related with the non-conservation of effective Planck mass, as experienced by travelling GWs. Indeed, we expect its RHS to be proportional to the rate of change of the effective Planck mass, a phenomenon already discussed in related settings, see e.g. [41, 46]. Eq. (3.14) provides a 'covariant' version of this result in the 
gauge we are adopting. This fact has interesting phenomenological consequences being related with observables associated with the GW luminosity distance, see e.g. [4147]. In the next Sections, we shall make this connection more explicit when analyzing specific models.

There can also be theories where the graviton number is not conserved (as theories with extra dimensions, see e.g. [10,12, 112-114] for recent studies in the framework of GW cosmology) which might be described in terms of effective scalar-tensor systems; in this case, a non-vanishing RHS of Eq. (3.14) controls the amount of graviton number non-conservation.

- Eq. (3.15) controls the evolution of the polarization tensor. If its RHS is non-vanishing, it implies that this quantity is not parallel transported along GW null geodesics. This is a novel effect, which again depends on the structure of the high-frequency EMT $t_{\mu \nu}$. Similar phenomena have been noticed in standard gravity working beyond a geometrical optics approximation in GR - see e.g. [50-53, 115, 116] - and might be related with lensing of GWs. Interestingly, we find that these effects can be present also at leading order in geometrical optics for certain scalar-tensor theories. We shall return in the next Sections on the physical implications of this result, when specializing to particular models.

Solving the independent Eqs. (3.14)-(3.15) allows one to determine the most general solution for $\mathcal{A}$ and for the transverse, normalized tensor $\mathbf{e}_{\mu \nu}$. Moreover, contracting Eq. (3.13) with $\bar{v}^{\mu}$, and using the gauge conditions Eq. (3.8) and Eq. (3.9), we find the following condition on the transverse vector

$$
2 k_{\rho} \bar{\nabla}^{\rho} \mathcal{C}_{\nu}=\mathcal{A}_{\mu \nu}\left[2 k^{\rho} \bar{\nabla}_{\rho} \bar{v}^{\mu}-\bar{v}^{\mu} \bar{\nabla}_{\rho} k^{\rho}\right]+\bar{v}^{\mu} t_{\mu \nu}
$$

This equation informs us that the vector $\mathcal{C}^{\mu}$ is in general not parallel transported along GW null geodesics (unless its RHS vanish). The RHS of Eq. (3.16) is made of two contributions: the first only depends on the background gradient $\bar{v}^{\mu}$ of the scalar field; the second depends on $t_{\mu \nu}$, hence its structure depends on the specific modified gravity model.

\subsection{On the decomposition of the energy-momentum tensor $t_{\mu \nu}$}

Even without relying on any specific scalar-tensor theory, we now have sufficient ingredients to pin down the general structure of the EMT for the systems we are interested in. In fact, it has to be: symmetric, transverse (in the sense that it has to satisfy Eq. (3.12)) and built with suitable combinations of the vectors $\left(\bar{v}^{\mu}, k^{\mu}, \mathcal{C}^{\mu}\right)$ and tensors $\left(\mathbf{e}_{\mu \nu}, \bar{g}_{\mu \nu}\right)$ as it is formed by metric fluctuations. Hence, it is necessarily of the form

$$
t_{\mu \nu} \equiv\left\{\tau^{(A)} \mathcal{A}_{\mu \nu}+\tau^{(B)}\left(k_{\mu} \mathcal{C}_{\nu}+k_{\nu} \mathcal{C}_{\mu}\right)+\tau^{(C)}\left[k_{\mu} \bar{v}_{\nu}+k_{\nu} \bar{v}_{\mu}-\bar{g}_{\mu \nu}\left(k_{\rho} \bar{v}^{\rho}\right)\right]+\tau^{(D)} k_{\mu} k_{\nu}\right\}
$$

since the four contributions proportional to the parameters $\tau^{(i)}$ are the only tensors with the desired properties. The last contribution proportional to $\tau^{(D)}$ will be associated with the self-induced GW energy momentum tensor at second order, a quantity that we shall not meet any more in this work, hence we set from now on $\tau^{(D)}=0$. In Eq. $(3.17), \tau^{(A)}, \tau^{(B)}$, $\tau^{(C)}$ are scalar functions of the space-time coordinates, that depend on the specific theory under consideration, on the smooth profiles of the quantities $\bar{\varphi}$ and $\bar{g}_{\mu \nu}$, and (possibly) on the 
trace of the polarization tensor and on the GW momentum $k^{\mu}$. In Section 4, when discussing explicit examples, we shall find realizations for each of the three contributions to Eq. (3.17).

Using the explicit decomposition Eq. (3.17), we can re-express the evolution equations for amplitude and polarization:

$$
\begin{aligned}
\bar{\nabla}_{\rho}\left(k^{\rho} \mathcal{A}^{2}\right) & =\mathcal{A}^{2} \tau^{(A)}-\tau^{(C)}(k \cdot \bar{v}) \mathcal{A}^{\mu \nu} \bar{g}_{\mu \nu} \\
2 k_{\rho} \bar{\nabla}^{\rho} \mathbf{e}_{\mu \nu} & =\tau^{(B)}\left(k_{\mu} \mathcal{C}_{\nu}+k_{\nu} \mathcal{C}_{\mu}\right)+\tau^{(C)}\left[k_{\mu} \bar{v}_{\nu}+k_{\nu} \bar{v}_{\mu}-\left(\bar{g}_{\mu \nu}-\mathbf{e}_{\mu \nu} \mathbf{e}\right)\left(k_{\rho} \bar{v}^{\rho}\right)\right]
\end{aligned}
$$

with the trace

$$
\mathbf{e}=\bar{g}^{\mu \nu} \mathbf{e}_{\mu \nu} .
$$

In terms of this EMT decomposition, the evolution Eq. (3.16) for the vector $\mathcal{C}_{\mu}=\bar{v}^{\nu} \mathcal{A}_{\mu \nu}$ reads

$$
2 k_{\rho} \bar{\nabla}^{\rho} \mathcal{C}_{\mu}=\mathcal{A}_{\mu \nu}\left[2 k^{\rho} \bar{\nabla}_{\rho} \bar{v}^{\mu}-\bar{v}^{\mu} \bar{\nabla}_{\rho} k^{\rho}\right]+\mathcal{C}_{\mu}\left[\tau^{(A)}+\tau^{(B)}(v \cdot k)\right]+k_{\mu}\left[\tau^{(B)}(\bar{v} \cdot \mathcal{C})+\bar{v}^{2} \tau^{(C)}\right]
$$

We then learn that the evolution equations for GW amplitude and polarization depend on different contributions to the EMT. The equation of evolution for the amplitude $\mathcal{A}$ can be further decomposed in a tensor and scalar part, as we are going to discuss in what next.

\subsection{Separating the spin-2 and spin-0 degrees of freedom}

So far, we made use of a polarization tensor $\mathcal{A}_{\mu \nu}$ that satisfy the gauge conditions Eq. (2.33). As scalar and tensor modes propagate at the same speed we assigned to both waves the same phase and, in the geometric optics limit, we showed that the associated wave vector is a null vector Eq. (3.10). This means that they are null waves, therefore they can be studied in the framework of null-tetrads as shown in the classic work of [117] (see also the textbook [111]).

In particular, in this formalism, there are six independent polarization modes which are dynamical or not according to the theory taken into account. Such modes correspond to two spin-2 modes, two scalar modes and two vector modes. In Appendix B we show how to build these independent polarizations and that in the theory Eq. (4.1) only three of them are dynamical. This is compatible with the fact that in the theory we are considering vectors are not dynamical and the scalar field is massless. Moreover, since $t_{\mu \nu}$ contains the rapid fluctuations of the fields as well, it will change after the removal of non-dynamical modes. In Appendix B we give a thorough explanation of how to treat these spurious modes. In particular we show that they decouple from the physical ones and how to build the first order stress energy tensor in terms only of the three physical polarizations. In this Section, thus, we consider only the spin- 2 and spin- 0 transverse modes and we call $t_{\mu \nu}^{\prime}$ the first order stress energy tensor after the removal of the spurious modes.

The three propagating modes present in the theory we are considering are the spin-2 transverse and traceless modes, represented by the polarization tensors $\left(\mathbf{e}_{\mu \nu}^{+}, \mathbf{e}_{\mu \nu}^{\times}\right)$, and the spin-0 transverse scalar mode described by $\mathbf{e}_{\mu \nu}^{S}$. Such polarization tensors are all normalized to 1, transverse as they have to satisfy Eq. (3.7) and orthogonal between each other. Their traces are

$$
\begin{aligned}
& \bar{g}^{\mu \nu} \mathbf{e}_{\mu \nu}^{+}=0, \\
& \bar{g}^{\mu \nu} \mathbf{e}_{\mu \nu}^{\times}=0, \\
& \bar{g}^{\mu \nu} \mathbf{e}_{\mu \nu}^{S}=\sqrt{2} .
\end{aligned}
$$


We also assume that the two spin-2 modes have the same amplitude, $\mathcal{A}^{T}$, as it should be in a non-parity violating theory. The more general case is considered in Appendix B.2. Hence, we decompose the tensor $\mathcal{A}_{\mu \nu}$ on the three polarizations as

$$
\mathcal{A}_{\mu \nu}=\mathcal{A} \mathbf{e}_{\mu \nu}=\mathcal{A}^{T}\left(\mathbf{e}_{\mu \nu}^{+}+\mathbf{e}_{\mu \nu}^{\times}\right)+\mathcal{A}^{S} \mathbf{e}_{\mu \nu}^{S}
$$

where

$$
\mathcal{A}=\sqrt{2\left(\mathcal{A}^{T}\right)^{2}+\left(\mathcal{A}^{S}\right)^{2}}
$$

The trace of Eq. (3.24) gives

$$
\mathbf{e}:=\bar{g}^{\mu \nu} \mathbf{e}_{\mu \nu}=\sqrt{2} \mathcal{A}^{S} / \mathcal{A},
$$

which states that the trace of the polarization is proportional to the amplitude of the spin- 0 mode, which we have hidden in the GW's polarization content because of our gauge choice. The first order stress-energy tensor without the gauge modes reads

$$
t_{\mu \nu}^{\prime}=\tau^{(A)} \mathcal{A}_{\mu \nu}-\sqrt{2} \tau^{(C)}\left(k_{\rho} \bar{v}^{\rho}\right) \mathbf{e}_{\mu \nu}^{S}
$$

where $\tau^{(A)}$ and $\tau^{(C)}$ are the ones defined in Eq. (3.17). This expression is derived in Appendix B.2. By plugging Eq. (3.24) into Eq. (3.13) and considering $t_{\mu \nu}^{\prime}$ as first-order stress energy tensor, we get

$$
2 k_{\rho} \bar{\nabla}^{\rho}\left(\mathcal{A}^{T} \mathbf{e}_{\mu \nu}^{+}+\mathcal{A}^{T} \mathbf{e}_{\mu \nu}^{\times}+\mathcal{A}^{S} \mathbf{e}_{\mu \nu}^{S}\right)+\left(\mathcal{A}^{T} \mathbf{e}_{\mu \nu}^{+}+\mathcal{A}^{T} \mathbf{e}_{\mu \nu}^{\times}+\mathcal{A}^{S} \mathbf{e}_{\mu \nu}^{S}\right)\left(\bar{\nabla}^{\rho} k_{\rho}\right)=t_{\mu \nu}^{\prime} .
$$

In Appendix B.2, starting from Eq. (3.28), we show how to separate the evolution of the scalar and tensor amplitudes, namely

$$
\begin{aligned}
& 2 k^{\rho} \bar{\nabla}_{\rho} \mathcal{A}^{S}+\mathcal{A}^{S} \bar{\nabla}_{\rho} k^{\rho}=t^{\prime \mu \nu} \mathbf{e}_{\mu \nu}^{S}=\tau^{(A)} \mathcal{A}^{S}-\sqrt{2}\left(\bar{v}^{\rho} k_{\rho}\right) \tau^{(C)}, \\
& 2 k^{\rho} \bar{\nabla}_{\rho} \mathcal{A}^{T}+\mathcal{A}^{T} \bar{\nabla}_{\rho} k^{\rho}=\frac{t^{\prime \mu \nu}}{2}\left(\mathbf{e}_{\mu \nu}^{+}+\mathbf{e}_{\mu \nu}^{\times}\right)=\tau^{(A)} \mathcal{A}^{T}
\end{aligned}
$$

So we obtain two equations for the amplitudes of the spin-0 and spin-2 modes generically coupled via the evolution of $t_{\mu \nu}^{\prime}$. We notice that the equation of $\mathcal{A}^{T}$ admits as solution $\mathcal{A}^{T}=0$ regardless of the form of the first order stress-energy tensor. On the contrary, the equation for $\mathcal{A}^{S}$ has a source term proportional to $\tau^{(C)}$. Thus, $\mathcal{A}^{S}=0$ is a solution only if $\tau^{(C)}$ is equal to zero or proportional to $\mathcal{A}^{S}$ itself. We will see that for the Lagrangian (4.1) braiding can play the role of source, while conformal couplings generates $\tau^{(C)} \propto \mathcal{A}^{S}$. In the case $\mathcal{A}^{S} \neq 0$ the previous two equations are equivalent to

$$
\begin{aligned}
& \bar{\nabla}_{\rho}\left(k^{\rho}\left(\mathcal{A}^{S}\right)^{2}\right)=\tau^{(A)}\left(\mathcal{A}^{S}\right)^{2}-\sqrt{2}\left(\bar{v}^{\rho} k_{\rho}\right) \mathcal{A}^{S} \tau^{(C)}, \\
& \bar{\nabla}_{\rho}\left(k^{\rho}\left(\mathcal{A}^{T}\right)^{2}\right)=\tau^{(A)}\left(\mathcal{A}^{T}\right)^{2} .
\end{aligned}
$$

Hence the EMT contributions to the evolution of the GW amplitude is in principle different for spin-2 and spin-0 modes. In both cases, we can have non-conservation of the spin-2 and spin-0 currents $\left(\mathcal{A}^{T} k^{\mu}\right)$ and $\left(\mathcal{A}^{S} k^{\mu}\right)$.

The solutions of Eq. (3.32) and Eq. (3.31) depend on the initial conditions. For example, one can expect that the initial amplitude of scalar components $\mathcal{A}^{S}$ to be smaller than 
the tensor one $\mathcal{A}^{T}$, thanks to screening mechanisms that reduce the size of scalar excitation around the source. For an explicit example where this happens, see [118]. This is an interesting topic to explore, that goes beyond the scope of this work.

After the removal of the spurious modes, as shown in Appendix B.2, Eq. (3.15) for the polarization tensor $\mathbf{e}_{\mu \nu}$ becomes

$$
k_{\rho} \bar{\nabla}^{\rho} \mathbf{e}_{\mu \nu}=\frac{1}{2 \mathcal{A}}\left[t_{\mu \nu}^{\prime}-\mathbf{e}_{\mu \nu}\left(t_{\rho \sigma}^{\prime} \mathbf{e}^{\rho \sigma}\right)\right]=\frac{\left(k_{\rho} \bar{v}^{\rho}\right) \tau^{(C)}}{\sqrt{2} \mathcal{A}}\left[\frac{\mathcal{A}^{S}}{\mathcal{A}^{2}} \mathcal{A}_{\mu \nu}-\mathbf{e}_{\mu \nu}^{S}\right]
$$

therefore $\mathbf{e}_{\mu \nu}$ is parallel transported or not according to whether the RHS of Eq. (3.33) vanishes or not. We will show that in the theory described by (4.1), the RHS of this equation is different from zero, so the total polarization is not parallel transported.

\section{An explicit scalar-tensor realization}

The geometrical optics framework developed in the previous Sections provides us with a general and flexible formalism that allows us to consistently treat the evolution of highfrequency GWs, and to acquire a transparent physical understanding of the results. Our formulas depend on the EMT tensor $t_{\mu \nu}$ which is controlled by the first derivatives of the high-frequency fields. To investigate our formalism in a concrete setting, we focus in this Section on a specific case of the scalar-tensor theory called kinetic gravity braiding [72], supplemented with a non-minimal coupling with the Ricci scalar. Its Lagrangian density can be expressed as

$$
\mathcal{L}^{(\text {tot })}=\mathcal{L}^{(F)}+\mathcal{L}^{(G)}+\mathcal{L}^{(K)}
$$

with

$$
\begin{aligned}
\mathcal{L}^{(F)} & =F(\varphi) R, \\
\mathcal{L}^{(G)} & =G(\varphi, X) \square \varphi, \\
\mathcal{L}^{(K)} & =K(\varphi, X),
\end{aligned}
$$

where $X=-\left(\partial_{\mu} \varphi \partial^{\mu} \varphi\right) / 2$. This is the most general subset of the Horndeski theory that leads to GW propagation at the speed of light, consistent with observational findings associated with the GW170817 event [119]. In order to apply the formalism we developed in the previous Sections, we focus on a set-up where we assume that the rapidly moving scalar fluctuations move with the speed of light. We further discuss this condition in Appendix A.

The Lagrangian $\mathcal{L}^{(F)}$ is related with the classic Brans-Dicke theory [120], and is common to many scalar-tensor theories that allow for a kinetic mixing between scalar and tensor fields in the Jordan frame. (Demixing can be obtained through a conformal transformation, at the price of introducing non-minimal couplings between scalar and matter degrees of freedom, that we prefer to avoid.) The Lagrangian $\mathcal{L}^{(G)}$ is a generalized cubic Galileon [59]. The contribution $\mathcal{L}^{(K)}$ corresponds instead to K-essence [58]. It would be interesting to explore further generalizations to DHOST theories [65-71] with the same property, or to consider scalar-tensor scenarios aimed to avoid constraints from graviton decay into dark energy [121123]. We plan to study such generalizations in future works. 
The energy-momentum tensor $T_{\mu \nu}$ associated $^{6}$ with the Lagrangian density Eq. (4.1) can be expressed as [62]

$$
T_{\mu \nu}^{(\mathrm{tot})}=T_{\mu \nu}^{(F)}+T_{\mu \nu}^{(G)}+T_{\mu \nu}^{(K)},
$$

with

$$
\begin{aligned}
T_{\mu \nu}^{(F)} & =\frac{F_{\varphi}}{F}\left(\nabla_{\mu} \nabla_{\nu} \varphi-g_{\mu \nu} \square \varphi\right)+\frac{F_{\varphi \varphi}}{F}\left(\nabla_{\mu} \varphi \nabla_{\nu} \varphi+2 X g_{\mu \nu}\right), \\
T_{\mu \nu}^{(G)} & =\frac{1}{2 F}\left(G_{X} \square \varphi \nabla_{\mu} \varphi \nabla_{\nu} \varphi+\nabla_{\mu} G \nabla_{\nu} \varphi+\nabla_{\nu} G \nabla_{\mu} \varphi-g_{\mu \nu} \nabla_{\lambda} G \nabla^{\lambda} \varphi\right), \\
T_{\mu \nu}^{(K)} & =\frac{1}{2 F}\left(K_{X} \nabla_{\mu} \varphi \nabla_{\nu} \varphi+K g_{\mu \nu}\right),
\end{aligned}
$$

The dynamics of the system is also controlled by the scalar equations of motion. We have proved that the relevant scalar equations are satisfied once the gravitational and the EMT conservation Eq. (2.51) are satisfied, hence we do not discuss the scalar equation any further.

We notice that $\mathcal{L}^{(G)}$ and $\mathcal{L}^{(F)}$ contain, respectively, derivative scalar self-couplings and non-minimal couplings of scalar to gravity, which lead to derivative contributions to the EMT involving metric fields. Hence, we expect them to give relevant contributions to the system evolution in the limit of geometrical optics. In order to extract the high frequency contributions to the linearized EMT tensor, i.e. $t_{\mu \nu}$ as defined in Eq. (3.4), one has to plug in the decompositions (2.2) and (2.4) into Eq. (4.5), use the gauge conditions discussed in Section 2.5.3 and then use the geometric optics Ansatz. Once this is done, one can organize the $1 / \epsilon$ contribution of the linearized EMT in the form of Eq. (3.17), that we re-write here

$$
t_{\mu \nu}^{(\text {tot })} \equiv\left\{\tau^{(A)} \mathcal{A}_{\mu \nu}+\tau^{(B)}\left(k_{\mu} \mathcal{C}_{\nu}+k_{\nu} \mathcal{C}_{\mu}\right)+\tau^{(C)}\left[k_{\mu} \bar{v}_{\nu}+k_{\nu} \bar{v}_{\mu}-\bar{g}_{\mu \nu}\left(k_{\rho} \bar{v}^{\rho}\right)\right]\right\} .
$$

For the model (4.1) the three functions $\tau^{(A)}, \tau^{(B)}$ and $\tau^{(C)}$ read

$$
\begin{aligned}
\tau^{(A)} & =-\frac{F_{\varphi}}{F}\left(k_{\rho} \bar{v}^{\rho}\right), \\
\tau^{(B)} & =\frac{F_{\varphi}}{F} \\
\tau^{(C)} & =-\frac{G_{X}}{2 F}\left(\bar{v}_{\rho} \mathcal{C}^{\rho}-\frac{\bar{v}^{2}}{2} \bar{g}^{\rho \sigma} \mathcal{A}_{\rho \sigma}\right)-\frac{F_{\varphi}}{2 F} \bar{g}^{\rho \sigma} \mathcal{A}_{\rho \sigma},
\end{aligned}
$$

Notice that, as anticipated above, only $\mathcal{L}^{F}$ and $\mathcal{L}^{G}$ contribute to the high-frequency EMT $t_{\mu \nu}$ and that

$$
\bar{g}^{\rho \sigma} \mathcal{A}_{\rho \sigma}=\sqrt{2} \mathcal{A}^{S},
$$

because of the trace properties of the polarization tensors. We can then apply the evolution equations derived in the previous Section to the present instance. We find the following results:

i) From Eq. (3.32) an evolution equation for the tensor component of the GW amplitude:

$$
\bar{\nabla}_{\rho}\left(k^{\rho}\left(\mathcal{A}^{T}\right)^{2}\right)=-\frac{F_{\varphi}}{F}\left(k_{\rho} \bar{v}^{\rho}\right)\left(\mathcal{A}^{T}\right)^{2} .
$$

\footnotetext{
${ }^{6}$ The total action for the system can contain additional smooth matter fields that can influence cosmological evolution. We do not consider them here, since we focus on GWs in the geometrical optics limit.
} 
ii) From Eq. (3.31) an evolution equation for the scalar component of the GW amplitude:

$$
\bar{\nabla}_{\rho}\left(k^{\rho}\left(\mathcal{A}^{S}\right)^{2}\right)=\frac{G_{X}}{2 F}\left(k_{\rho} \bar{v}^{\rho}\right) \mathcal{A}^{S}\left(\sqrt{2} \bar{v}_{\rho} \mathcal{C}^{\rho}-\bar{v}^{2} \mathcal{A}^{S}\right)
$$

iii) From Eq. (3.33) an evolution equation for the polarization tensor:

$$
2 k_{\rho} \bar{\nabla}^{\rho} \mathbf{e}_{\mu \nu}=-\left[\frac{G_{X}}{2 F}\left(\bar{v}_{\rho} \mathcal{C}^{\rho}-\frac{\bar{v}^{2}}{\sqrt{2}} \mathcal{A}^{S}\right)+\frac{F_{\varphi}}{\sqrt{2} F} \mathcal{A}^{S}\right] \frac{\left(k_{\rho} \bar{v}^{\rho}\right)}{\sqrt{2} \mathcal{A}}\left[\frac{\mathcal{A}^{S}}{\mathcal{A}^{2}} \mathcal{A}_{\mu \nu}-\mathbf{e}_{\mu \nu}^{S}\right]
$$

iv) By contracting Eq. (3.28) with $\bar{v}^{\nu}$, an evolution equation for the vector $\mathcal{C}_{\mu}$ :

$$
\begin{aligned}
2 k^{\rho} \bar{\nabla}_{\rho} \mathcal{C}_{\mu}= & \mathcal{A}_{\mu \nu}\left[2 k^{\rho} \bar{\nabla}_{\rho} \bar{v}^{\mu}-\bar{v}^{\mu} \bar{\nabla}_{\rho} k^{\rho}\right]-\frac{F_{\varphi}}{F}\left(\bar{v}^{\rho} k_{\rho}\right)\left[\mathcal{C}_{\mu}-\frac{\mathcal{A}^{S}}{\sqrt{2}} \bar{v}^{\nu} \mathbf{e}_{\mu \nu}^{S}\right]+ \\
& +\frac{G_{X}\left(\bar{v}^{\rho} k_{\rho}\right)}{2 F}\left[\sqrt{2} \bar{v}^{\rho} \mathcal{C}_{\rho}-\bar{v}^{2} \mathcal{A}^{S}\right] \bar{v}^{\nu} \mathbf{e}_{\mu \nu}^{S}
\end{aligned}
$$

The four equations above are the most general result obtained in this work. They describe the evolution of both the GW scalar and tensor modes in the considered theory of gravity. They show that the GW propagation depends on the assumed scalar-tensor theory (through the dependence on the functions $F_{\varphi}, G_{X}$ ), and on the background quantities $\bar{g}_{\mu \nu}$ and $\bar{v}^{\mu}=\bar{\nabla}^{\mu} \bar{\varphi}$. Moreover, from Eq. (4.15) we note that the evolution of the scalar amplitude $\mathcal{A}^{S}$ cannot be decoupled from that of the tensor amplitude $\mathcal{A}^{T}$, due to the presence of the total amplitude $\mathcal{A}$ in Eq. (4.15) $\left(\mathcal{C}_{\mu}=\bar{v}^{\nu} \mathcal{A}_{\mu \nu}\right)$. However, Eq. (4.14) shows that the tensor amplitude $\mathcal{A}^{T}$ does not depend on $\mathcal{A}^{S}$. In addition, Eq. (4.16) implies that the tensor and scalar modes of polarization are in general coupled one to each other.

The evolution equation for the tensor amplitude, Eq. (4.14) is proportional to $F_{\varphi}$. This is a quantity that in a cosmological setting is associated with the time-dependence of the effective Planck mass. In theories as the ones we consider here - with second order equations of motion, and GW unit speed - the failure of conservation of the current $\left(\mathcal{A}^{T}\right)^{2} k^{\mu}$ is associated with the rate of change of the effective Planck mass, a result well known in the literature (see e.g. the discussion in the recent [41] and references therein).

The evolution equation for the scalar part of the amplitude, Eq. (4.15) is more complex, and depends both on $F$ and $G_{X}$. As previously stated, in the absence of braiding $\mathcal{A}^{S}=0$ is a solution of its evolution equation (3.29). This implies that, if the scalar mode is not produced by a source, then it is absent. This could be the case, for instance, if screening at the source is very effective.

In Eq. (4.16), the RHS quantifies the failure of the GW polarization tensor of being parallel transported along the GW geodesics. We can see that, only in the specific sub-case in which $G_{X}=0$ and $\mathcal{A}^{S}=0$, the polarization tensor is parallel transported. In fact, only when these two conditions are met $\tau^{(C)}=0$.

\section{GW propagation on a cosmological space-time}

One feature of the geometrical optics formalism we developed in the previous Sections is that it can be applied to GWs travelling over an arbitrary space-time, as long as the scale 
of variation of the background geometry is well larger than the GW wavelength. In this Section, we specialize to GWs travelling over a perturbed FRW universe, and apply the formulas derived in the previous Section for a specific scalar-tensor model. Using the Cosmic Rulers formalism [108] (see the discussion in the Introduction) we will show how to derive an expression for the GW luminosity distance from the analysis of the amplitude of the tensor modes, whose dynamics is governed by Eq. (4.14).

We report here the main equations we will use in this Section. As we wish to focus only on tensor modes, these are

$$
\begin{gathered}
\bar{\nabla}_{\rho}\left(k^{\rho}\left(\mathcal{A}^{T}\right)^{2}\right)=-\frac{F_{\varphi}}{F}\left(k_{\rho} \bar{v}^{\rho}\right)\left(\mathcal{A}^{T}\right)^{2} \\
2 k_{\rho} \bar{\nabla}^{\rho} \mathbf{e}_{\mu \nu}=-\left[\frac{G_{X}}{2 F}\left(\bar{v}_{\rho} \mathcal{C}^{\rho}-\frac{\bar{v}^{2}}{\sqrt{2}} \mathcal{A}^{S}\right)+\frac{F_{\varphi}}{\sqrt{2} F} \mathcal{A}^{S}\right] \frac{\left(k_{\rho} \bar{v}^{\rho}\right)}{\sqrt{2} \mathcal{A}}\left[\frac{\mathcal{A}^{S}}{\mathcal{A}^{2}} \mathcal{A}_{\mu \nu}-\mathbf{e}_{\mu \nu}^{S}\right] \\
2 k^{\rho} \bar{\nabla}_{\rho} \mathcal{C}_{\mu}=\mathcal{A}_{\mu \nu}\left[2 k^{\rho} \bar{\nabla}_{\rho} \bar{v}^{\mu}-\bar{v}^{\mu} \bar{\nabla}_{\rho} k^{\rho}\right]-\frac{F_{\varphi}}{F}\left(\bar{v}^{\rho} k_{\rho}\right)\left[\mathcal{C}_{\mu}-\frac{\mathcal{A}^{S}}{\sqrt{2}} \bar{v}^{\nu} \mathbf{e}_{\mu \nu}^{S}\right]+ \\
+\frac{G_{X}\left(\bar{v}^{\rho} k_{\rho}\right)}{2 F}\left[\sqrt{2} \bar{v}^{\rho} \mathcal{C}_{\rho}-\bar{v}^{2} \mathcal{A}^{S}\right] \bar{v}^{\nu} \mathbf{e}_{\mu \nu}^{S}
\end{gathered}
$$

We wish to stress that, in general, the GW polarization is not parallely transported in modified gravity. However, in order to keep the discussion simpler, when studying the polarization tensor in this Section we will choose the simplified scenario $G_{X}=0$ and assume that the scalar wave is not produced at the source in order to set also $\mathcal{A}^{S}=0$. Eq. (5.2) and Eq. (5.3) inform us that for the case of an unperturbed FRW space-time, we are allowed to make the choice $\mathcal{C}_{\mu}=0$. Indeed, in a homogeneous FRW space-time, the scalar background configuration depends only time only, and $\bar{v}^{\mu} \propto \delta_{0}^{\mu}$. If $\mathcal{C}^{\mu}=0$, then the polarization tensors satisfy the condition $\mathbf{e}_{\mu 0}=0$, and they have non-vanishing spatial components only. Since $\mathbf{e}_{i j}$ is transverse to the GW direction, we can without loss of generality choose a frame in which the GW propagates along the $z$-direction, and $\mathbf{e}_{i j}$ has non-vanishing components in the $(x, y)$-directions only. A simple calculation shows that, in this case, the first term in the RHS of Eq. (5.3) vanishes. This implies that the choice $\mathcal{C}_{\mu}=0$ satisfies this equation, and also implies that the RHS of Eq. (5.2) vanishes. Hence, when focusing on an homogeneous, unperturbed FRW space-time, if only the GW tensor components propagate and $\mathcal{C}^{\mu}=0$, the geometrical optics equations prescribe that the polarization tensor is parallel propagated along geodesics as the RHS of Eq. (5.2) is zero in this case. For the case of a perturbed space-time, instead, the situation is more complex, since all quantities depend both on time and space, and the previous arguments do not hold.

We use as background (i.e. slowly varying) space-time quantities

$$
\begin{aligned}
& d s_{\bar{g}}^{2}=\bar{g}_{\mu \nu} d x^{\mu} d x^{\nu}=a^{2}(\eta)\left[-(1+2 \phi) d \eta^{2}+(1-2 \psi) \delta_{i j} d x^{i} d x^{j}\right], \\
& \bar{\varphi}=\varphi_{0}(\eta)+\delta \varphi\left(x^{\mu}\right) .
\end{aligned}
$$

All fluctuations appearing in the previous Eqs. (5.4) and (5.5) are assumed to have long wavelengths, much larger than the high frequency GW modes that travel on such space-time. Cosmological perturbations have the effect to alter the estimate of astrophysical parameters derived for the binary system. For example, the inferred luminosity distance of the source of the GWs will differ from the actual one because of the presence of inhomogeneities. It is important to clarify whether there are degeneracies between these effects and modified gravity. The propagation of short-wavelength GWs over a metric which includes long-wavelength 
scalar fluctuations has been also investigated in [106], with the specific purpose of analyzing some consequences of a novel proposal for evading GW170817 constraints on scalar-tensor scenarios.

\subsection{Cosmic rulers for gravitational waves}

In order to study how cosmological perturbations affect the propagation of gravitational waves we follow [107], but in the more general scalar-tensor theory presented in the previous Section. In [107] the authors use the so called Cosmic Rulers formalism [108] to compute the correction to the luminosity distance of the spiraling binary. This formalism has been first formulated for electromagnetic radiation [124], [108] but it can be immediately extended to gravitational radiation in the geometrical optics limit. In the Cosmic Rulers formalism, the observer frame, called the Redshift-GW frame (RGW), is used as reference system. Such frame is different from the real-frame (or equivalently called the physical-space) which is the frame where we computed Eqs. (5.1), (5.2) and (5.3).

Since we will use as background metric Eq. (5.4), it is convenient to perform a conformal transformation,

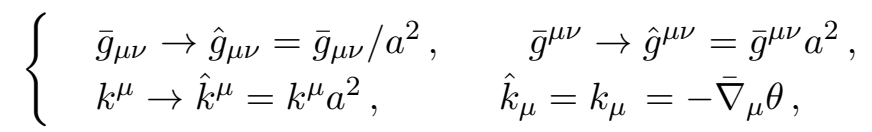

in this way $\hat{g}_{\mu \nu}=\eta_{\mu \nu}+\delta \hat{g}_{\mu \nu}$. Under the transformation (5.6) the connection coefficients transform as

$$
\begin{aligned}
\Gamma_{\nu \rho}^{\mu} & =\frac{1}{2 a^{2}} \hat{g}^{\mu \alpha}\left(\left(a^{2} \hat{g}_{\nu \alpha}\right)_{, \rho}+\left(a^{2} \hat{g}_{\rho \alpha}\right)_{, \nu}-\left(a^{2} \hat{g}_{\nu \rho}\right)_{, \alpha}\right)= \\
& =\hat{\Gamma}_{\nu \rho}^{\mu}+\frac{1}{2}\left(\delta_{\nu}^{\mu} \partial_{\rho} \ln a^{2}+\delta_{\rho}^{\mu} \partial_{\nu} \ln a^{2}-\delta_{\nu \rho} \hat{g}^{\mu \alpha} \partial_{\alpha} \ln a^{2}\right),
\end{aligned}
$$

where $\hat{\Gamma}_{\nu \rho}^{\mu}$ is the connection symbol associated to $\hat{g}_{\mu \nu}$.

In this Section we use the notation in which barred quantities belong to the RGW-frame, while unbarred quantities belong to the physical space. As we performed the conformal transformation (5.6) and decomposed the scalar field as (5.5) there is no confusion with the objects defined in the previous Sections.

We define $x^{\mu}(\chi)$ as the comoving coordinate in the real frame, where $\chi$ is the comoving distance to the observer. Using the notation just introduced, $\bar{x}^{\mu}(\bar{\chi})$ is the coordinate in the RGW-frame. In the RGW-frame we use coordinates that flatten our past gravitational wave-cone, therefore the geodesic of the emitted gravitational waves in the RGW-frame is given by

$$
\bar{x}^{\mu}(\bar{\chi})=(\bar{\eta}, \overline{\mathbf{x}})=\left(\eta_{0}-\bar{\chi}, \bar{\chi} \hat{\mathbf{n}}\right),
$$

where $\eta_{0}$ is the conformal time at observation, $\bar{\chi}(z)$ is the comoving distance to the observed redshift in the observer frame and $\hat{\mathbf{n}}$ is the observed direction of arrival in the sky, i.e. $\hat{n}^{i}=\bar{x}^{i} / \bar{\chi}=\delta^{i j}\left(\partial \bar{\chi} / \partial \bar{x}^{j}\right)$. The observed coordinate will be different from the one in the real frame and the total derivative along the part gravitational wave-cone is

$$
\frac{\mathrm{d}}{\mathrm{d} \bar{\chi}}=-\frac{\partial}{\partial \bar{\eta}}+\hat{n}^{i} \frac{\partial}{\partial \bar{x}^{i}} .
$$

It is convenient to define parallel and perpendicular projectors operator with respect the observed line-of-sight direction. As shown in $[107,108]$, for any spatial vector $B_{i}$ and tensor $A_{i j}$, in the RGW-space we have: 
1. $A_{\|}=\hat{n}^{i} \hat{n}^{j} A_{i j}$

2. $B_{\perp}^{i}=\mathcal{P}^{i j} B_{j}=B^{i}-\hat{n}^{i} B_{\|}$

3. $\bar{\partial}_{\|}=\hat{n}^{i} \bar{\partial}_{i}$

4. $\bar{\partial}_{\|}^{2}=\bar{\partial}_{\|} \bar{\partial}_{\|}$

5. $\bar{\partial}_{\perp i}=\mathcal{P}_{i}^{j}=\bar{\partial}_{i}-\hat{n}_{i} \bar{\partial}_{\|}$

6. $\bar{\partial}_{i} \hat{n}^{j}=\bar{\partial}_{i}\left(\frac{\bar{x}^{j}}{\bar{\chi}}\right)=\frac{1}{\bar{\chi}}\left(\delta_{i}^{j}-\frac{\bar{x}^{j}}{\bar{\chi}} \frac{\partial \bar{\chi}}{\partial \bar{x}^{i}}\right)=\frac{1}{\bar{\chi}}\left(\delta_{i}^{j}-\hat{n}^{j} \hat{n}_{i}\right)=\frac{1}{\bar{\chi}} \mathcal{P}_{i}^{j}$

7. $\frac{\mathrm{d}}{\mathrm{d} \bar{\chi}} \partial_{\perp}^{i}=\bar{\partial}_{\perp}^{i} \frac{\mathrm{d}}{\mathrm{d} \bar{\chi}}-\frac{1}{\bar{\chi}} \partial_{\perp}^{i}$

8. $\frac{\partial B^{i}}{\partial \bar{x}^{j}}=\bar{\partial}_{j \perp} B_{\perp}^{i}+\hat{n}_{j} \bar{\partial}_{\|} B_{\perp}^{i}+\frac{1}{\bar{\chi}} \mathcal{P}_{i}^{j} B_{\|}+\hat{n}^{i}\left[\bar{\partial}_{j \perp}+\hat{n}_{j} \bar{\partial}_{\|}\right] B_{\|}$

where we have used $\bar{\partial}_{i}=\partial / \partial \bar{x}^{i}$ and $\mathcal{P}_{j}^{i}=\delta_{j}^{i}-\hat{n}^{i} \hat{n}_{j}$. Note also that partial derivatives in the RGW-frame do not commute as shown in [108].

We define $\bar{k}^{\mu}$ as the null geodesic vector in the redshift frame at zeroth order,

$$
\bar{k}^{\mu}=\frac{\mathrm{d} \bar{x}^{\mu}}{\mathrm{d} \bar{\chi}}=(-1, \hat{\mathbf{n}}),
$$

which satisfies $\mathrm{d} \bar{k}^{\mu} / \mathrm{d} \bar{\chi}=0$.

Our aim is to compute GW observables in the RGW-space. In the previous section we derived the evolution equations of the quantities related to the GWs in the real frame, therefore we need to build a map to relate the two frames. We do so relying on the fact that the difference between the real-frame and the observer-frame is due to the presence of the linear perturbations describing the LSS present in the Universe. In fact, if the Universe was homogeneous and isotropic then, after the conformal transformation (5.6), the RGWspace would coincides with the real-frame. We define $\delta \chi$ to be the difference of the affine parameters $\chi$ and $\bar{\chi}$, so that

$$
\chi=\bar{\chi}+\delta \chi
$$

in this way the relation between the coordinates of the geodesic in the real-frame and the RGW-frame is given by

$$
\begin{aligned}
x^{\mu}(\chi)=x^{\mu}(\bar{\chi}+\delta \chi) & =x^{\mu}(\bar{\chi})+\frac{\mathrm{d} \bar{x}^{\mu}}{\mathrm{d} \bar{\chi}} \delta \chi= \\
& =\bar{x}^{\mu}(\bar{\chi})+\delta x^{\mu}(\bar{\chi})+\frac{\mathrm{d} \bar{x}^{\mu}}{\mathrm{d} \bar{\chi}} \delta \chi=\bar{x}^{\mu}(\bar{\chi})+\delta x^{\mu}(\bar{\chi})+\bar{k}^{\mu} \delta \chi
\end{aligned}
$$

where we used Eq. (5.10) in the last step and introduced $\delta x^{\mu}(\bar{\chi})$ which is the perturbation to the graviton's geodesics at fixed affine parameter, i.e. $\delta x^{\mu}(\bar{\chi}):=x^{\mu}(\bar{\chi})-\bar{x}^{\mu}(\bar{\chi})$. We define the total correction to the GW's geodesic as

$$
\Delta x^{\mu}(\bar{\chi}):=\delta x^{\mu}(\bar{\chi})+\bar{k}^{\mu} \delta \chi .
$$


so that the mapping between $x^{\mu}(\chi)$ and $\bar{x}^{\mu}(\bar{\chi})$ is given by

$$
x^{\mu}(\chi)=\bar{x}^{\mu}(\bar{\chi})+\Delta x^{\mu}(\bar{\chi})
$$

as also discussed in [108]. The correction $\Delta x^{\mu}(\bar{\chi})$ has two contributions: one at fixed observed-comoving distance, $\delta x^{\mu}(\bar{\chi})$, and the other one proportional to $\delta \chi$ in the direction of $\bar{k}^{\mu}$. The first contribution accounts for the perturbation of the GW's geodesics at $\bar{\chi}$ due to the presence of linear structure in the Universe, while the second one for the difference between the affine parameters of the geodesics in the two frames. In practice, using $\bar{\chi}$ instead of $\chi$ makes us "misread" the value of the affine parameter along the GW's geodesics at which we compute a certain quantity. This is actually true for all of the quantities characterizing the propagating GW (amplitude, phase and polarization) and the dark energy field, and not only the geodesics it follows. Hence, from now on, the symbol $\Delta$ stands for the sum of both these two kinds of contribution.

The wave-vector is defined as the vector tangent to the GW geodesic in the real-space $x^{\mu}(\chi)$, i.e. $\hat{k}^{\mu}=\mathrm{d} x^{\mu}(\chi) / \mathrm{d} \chi$. After some manipulations it is possible to see that it is given by

$$
\hat{k}^{\mu}=\frac{\mathrm{d} x^{\mu}(\chi)}{\mathrm{d} \chi}=\frac{\mathrm{d} \bar{\chi}}{\mathrm{d} \chi} \frac{\mathrm{d} x^{\mu}(\chi)}{\mathrm{d} \bar{\chi}}=\left(1-\frac{\mathrm{d} \delta \bar{\chi}}{\mathrm{d} \chi}\right) \frac{\mathrm{d}}{\mathrm{d} \bar{\chi}}\left(\bar{x}^{\mu}(\bar{\chi})+\Delta x^{\mu}(\bar{\chi})\right)=\bar{k}^{\mu}+\frac{\mathrm{d} \delta x^{\mu}}{\mathrm{d} \bar{\chi}}(\bar{\chi}),
$$

at linear order. We notice that the correction to $\hat{k}^{\mu}$ is only of the first kind, i.e. $\Delta \hat{k}^{\mu}(\bar{\chi})=$ $\delta \hat{k}^{\mu}(\bar{\chi})$, and it does not show any contribution $\propto \bar{k}^{\mu} \delta \chi$, as it was instead the case for $\Delta x^{\mu}(\bar{\chi})$. Therefore, we can find $\delta k^{\mu}$ through

$$
\hat{k}^{\mu}=\bar{k}^{\mu}+\delta k^{\mu}=\bar{k}^{\mu}+\frac{\mathrm{d} \delta x^{\mu}}{\mathrm{d} \bar{\chi}},
$$

which leads to

$$
\delta k^{\mu}=\frac{\mathrm{d} \delta x^{\mu}(\bar{\chi})}{\mathrm{d} \bar{\chi}}:=(\delta \nu(\bar{\chi}), \delta \mathbf{n}(\bar{\chi}))
$$

where $\delta \nu$ represents the correction to the frequency of the wave, and $\delta n^{i}$ the one to the direction of arrival. The wave-vector in the real-space is given by

$$
\hat{k}^{\mu}(\bar{\chi})=\left(-1+\delta \nu(\bar{\chi}), \hat{n}^{i}+\delta n^{i}(\bar{\chi})\right) .
$$

We point out also that the correction $\delta k^{\mu}$ satisfies the linearized geodesics equation and, in the next Section, we will use this fact to find $\delta \nu$ and $\delta n^{i}$ as a function of the gravitational potentials in Poisson's gauge. We can obtain $\delta x^{\mu}$ upon integration of Eq. (5.17)

$$
\delta x^{0}(\bar{\chi})=\int_{0}^{\bar{\chi}} \mathrm{d} \tilde{\chi} \delta \nu(\tilde{\chi}) \quad \text { and } \quad \delta x^{i}(\bar{\chi})=\int_{0}^{\bar{\chi}} \mathrm{d} \tilde{\chi} \delta n^{i}(\tilde{\chi})
$$

with the boundary condition $\delta x_{o}^{\mu}:=\delta x^{\mu}(\bar{\chi}=0)=0$ set at the observer position $\bar{\chi}=0$. The real-space scale factor is given by

$$
\frac{a\left(x^{0}(\chi)\right)}{\bar{a}}=\frac{a\left(\bar{x}^{0}+\Delta x^{0}\right)}{\bar{a}}=1+\Delta \ln a=1+\mathcal{H} \Delta x^{0},
$$


where $\bar{a}=a\left(\bar{x}^{0}\right)^{7}$.

We restrict ourselves to the local wave zone where the GW's wavelength is small with respect to the comoving distance from the observer. In this portion of the space-time we introduce the frame of reference of a comoving observer, described by the tetrad $e_{\hat{a}}^{\mu}$, which we extend until the source position. The time-like vector of the orthonormal basis can be chosen using the four-velocity $u^{\mu}$ of the observer

$$
u_{\mu}=e_{\hat{0} \mu}=a E_{\hat{0} \mu} \quad \text { and } \quad u^{\mu}=e_{\hat{0}}^{\mu}=a^{-1} E_{\hat{0}}^{\mu},
$$

where $E_{\mu}^{\hat{a}}$ is the tetrad in the comoving frame. The other components are constructed using the relations

$$
\hat{g}_{\mu \nu}=\eta_{\hat{a} \hat{b}} E_{\mu}^{\hat{a}} E_{\nu}^{\hat{b}}, \quad \eta^{\hat{a} \hat{b}}=\hat{g}_{\mu \nu} E_{\hat{a}}^{\mu} E_{\hat{b}}^{\nu}, \quad \hat{g}^{\mu \nu} E_{\mu}^{\hat{a}}=E^{\hat{a} \nu}, \quad \eta_{\hat{a} \hat{b}} E_{\mu}^{\hat{a}}=E_{\hat{b} \mu} .
$$

At linear order in Poisson's gauge $u_{\mu}=-a\left(1+\phi,-v_{i}\right)$, so that the comoving tetrad is given by

$$
E_{\hat{0} \mu}=\left(\begin{array}{c}
-1-\phi \\
v_{i}
\end{array}\right), \quad E_{\hat{a} 0}=-v_{\hat{a}} \quad \text { and } \quad E_{\hat{a} i}=\delta_{\hat{a} i}(1-\psi) .
$$

We can use this tetrad to compute the correction of the scale factor in Eq. (5.20). Calling "e" and "o" the emitted and observed positions, respectively, and denoting the GW frequency as $f$, the scale factor is related to the observed redshift as

$$
1+z=\frac{f_{e}}{f_{o}}=\frac{\left.\left(e_{\hat{0} \mu} k^{\mu}\right)\right|_{e}}{\left(e_{\hat{0} \mu} k^{\mu}\right)_{o}}=\frac{a_{0}}{a\left(\chi_{e}\right)} \frac{\left.\left(E_{\hat{0} \mu} \hat{k}^{\mu}\right)\right|_{e}}{\left(E_{\hat{0} \mu} \hat{k}^{\mu}\right)_{o}} .
$$

Setting $a_{o}=\bar{a}_{o}=1$ and $\left.\left(E_{\hat{0} \mu} k^{\mu}\right)\right|_{o}=1$, the previous equation becomes

$$
1+z=\frac{\left.\left(E_{\hat{0} \mu} \hat{k}^{\mu}\right)\right|_{e}}{a\left(\chi_{e}\right)}=\frac{\left.\left(E_{\hat{0} \mu} \hat{k}^{\mu}\right)^{(0)}\right|_{e}+\left.\left(E_{\hat{0} \mu} \hat{k}^{\mu}\right)^{(1)}\right|_{e}}{\bar{a}(1+\Delta \ln a)}=(1+z) \frac{1+\left(E_{\hat{0} \mu} \hat{k}^{\mu}\right)^{(1)}}{1+\Delta \ln a},
$$

where we used $\bar{a}=1 /(1+z)$. Hence

$$
\Delta \ln a=\left.\left(E_{\hat{0} \mu} \hat{k}^{\mu}\right)^{(1)}\right|_{e}=-E_{\hat{0} 0}^{(1)}+\hat{n}^{i} E_{\hat{0} i}^{(1)}-\delta \nu .
$$

\subsection{Gravitational waves in the observer frame}

In this Section we follow the same prescription developed in [107], and we extend their results by including modified gravity effects. In particular, we compute the effects of largescale structures on GW's waveforms (e.g. the phase and the amplitude) of the GW in MG accounting for lensing, Sachs-Wolfe, integrated Sachs-Wolfe, time delay and volume effects.

\subsubsection{The GW phase}

We showed that in the theory of gravity considered, the wave-vector is null and tangent to a null geodesic of the background space-time. After the conformal transformation defined in Eq. (5.6), the wave-vector in the conformal space satisfies

$$
\hat{k}^{\mu} \hat{k}_{\mu}=0,
$$

\footnotetext{
${ }^{7}$ In principle we should include the perturbation of the scale factor at observation $\delta a_{o}=a_{o}-1$. This extra term is an additive constant that we neglect for simplicity. For further details see, e.g., [92]).
} 
and using $\hat{k}_{\mu}=k_{\mu}=-\bar{\nabla}_{\mu} \theta$ we find

$$
0=\hat{k}^{\mu} \bar{\nabla}_{\mu} \theta=\frac{\mathrm{d} \theta}{\mathrm{d} \chi} .
$$

We wish to expand the equation above at linear order to find the effects of the structure present in the Universe on the phase of GWs. In order to do so we keep in mind that, when expanding to first order, every quantity gets two types of contribution: one at fixed comoving distance $\bar{\chi}$, denoted with $\delta$ and one that accounts for the due correction of the affine parameter, therefore proportional to $\delta \chi$. Accordingly, if $\bar{\theta}(\bar{\chi})$ is the phase of the wave at zero order, i.e. the one in the RGW-frame, we define $\delta \theta(\bar{x}(\bar{\chi}))=\theta(\bar{x}(\bar{\chi}))-\bar{\theta} \bar{x}(\bar{\chi}))$ so that the total correction to the phase is

$$
\begin{aligned}
\theta(x) & =\theta(\bar{x}+\Delta x)=\theta(\bar{x}(\bar{\chi}))+\Delta x^{\mu} \bar{\partial}_{\mu} \bar{\theta}(\bar{x}(\bar{\chi}))=\bar{\theta}(\bar{x}(\bar{\chi}))+\delta \theta(\bar{x}(\bar{\chi}))+\Delta x^{\mu} \bar{\partial}_{\mu} \bar{\theta}(\bar{x}(\bar{\chi}))= \\
& =\bar{\theta}(\bar{x}(\bar{\chi}))+\delta \theta(\bar{x}(\bar{\chi}))-\Delta x^{\mu} \bar{k}_{\mu}=\bar{\theta}(\bar{x}(\bar{\chi}))+\Delta \theta(\bar{x}(\bar{\chi}))
\end{aligned}
$$

where we used that at zero order $\bar{k}_{\mu}=-\bar{\partial}_{\mu} \bar{\theta}$. We defined $\Delta \theta(\bar{x}(\bar{\chi})):=\delta \theta(\bar{x}(\bar{\chi}))-\Delta x^{\mu} \bar{k}_{\mu}$ as the sum of the two contributions to the correction of the phase.

Using this prescription and Eqs. (5.14) we can expand at linear order Eq. (5.28) as

$$
\begin{aligned}
0=\frac{\mathrm{d} \theta\left(x^{\mu}(\chi)\right)}{\mathrm{d} \chi} & =\frac{\mathrm{d}}{\mathrm{d} \chi} \theta\left(\bar{x}^{\mu}+\Delta x^{\mu}\right)=\left(1-\frac{\mathrm{d} \delta \bar{\chi}}{\mathrm{d} \chi}\right) \frac{\mathrm{d}}{\mathrm{d} \bar{\chi}}(\bar{\theta}(\bar{x})+\Delta \theta(\bar{x}))= \\
& =\frac{\mathrm{d} \bar{\theta}}{\mathrm{d} \bar{\chi}}+\frac{\mathrm{d} \delta \theta}{\mathrm{d} \bar{\chi}}+\delta k^{\mu} \bar{\partial}_{\mu} \bar{\theta}+\delta x^{\mu} \frac{\mathrm{d}}{\mathrm{d} \bar{\chi}} \bar{\partial}_{\mu} \bar{\theta}=\frac{\mathrm{d} \bar{\theta}}{\mathrm{d} \bar{\chi}}+\frac{\mathrm{d} \delta \theta}{\mathrm{d} \bar{\chi}}-\delta k^{\mu} \bar{k}_{\mu},
\end{aligned}
$$

where in the last step we used that $-\bar{\partial}_{\mu} \bar{\theta}=\bar{k}_{\mu}$ and $d \bar{k}^{\mu} / d \bar{\chi}=0$. We can extract the zero and first order contributions from the equation above as they have to be satisfied separately,

$$
\frac{\mathrm{d} \bar{\theta}}{\mathrm{d} \bar{\chi}}=0 \quad \text { and } \quad \frac{\mathrm{d} \delta \theta}{\mathrm{d} \bar{\chi}}=\delta k^{\mu} \bar{k}_{\mu} .
$$

The last equation allows us to get $\delta \theta$ from $\delta k_{\mu}$ upon integration

$$
\delta \theta(\bar{\chi})=\delta \theta_{o}+\int_{0}^{\bar{\chi}} d \chi\left(\delta \nu+\delta n_{\|}\right)
$$

where $\delta \theta_{o}$ is the value of $\delta \theta$ at the observer position who is located at $\bar{\chi}=0$. The full correction to the phase, $\Delta \theta(\bar{x}(\bar{\chi}))$, is then given by

$$
\Delta \theta(\bar{x}(\bar{\chi}))=\delta \theta(\bar{x}(\bar{\chi}))-\Delta x^{\mu} \bar{k}_{\mu}=\delta \theta(\bar{x}(\bar{\chi}))-\Delta x^{0} \bar{k}_{0}-\Delta x^{i} \bar{k}_{i}=\delta \theta-\left(\Delta x^{0}+\Delta x_{\| \mid}\right) .
$$

since $\bar{k}_{0}=\eta_{\mu 0} \bar{k}^{\mu}=+1$. We can define $T$ as

$$
T=-\left(\Delta x^{0}+\Delta x_{\|}\right)=-\left(\delta x^{0}+\delta x_{\|}\right),
$$

therefore

$$
\Delta \theta(\bar{\chi})=\delta \theta(\bar{\chi})+T=\left.\delta \theta\right|_{o}+\int_{0}^{\bar{\chi}} d \chi\left(\delta \nu+\delta n_{\|}\right)+T=\left.\delta \theta\right|_{o},
$$

where we used Eq. (5.19) and Eq. (5.33) in the last equality. This result is in agreement with [107]. 


\subsubsection{The GW amplitude}

In the previous section we derived the evolution equation of the amplitude of the tensor part of the GW in the most general Horndeski theory satisfying the constraint $c_{T}=1$ and in which the scalar wave propagates at the same speed of the tensor modes. The amplitude $\mathcal{A}^{T}$ of waves is inversely proportional to the luminosity distance of the spiraling binary, and thus we are interested in deriving the expression of the observed amplitude. To this aim, it is useful to rewrite Eq. (4.14) as

$$
k^{\rho} \bar{\nabla}_{\rho} \ln \left(\mathcal{A}^{T}\right)=-\frac{1}{2}\left(\bar{\nabla}^{\rho} k_{\rho}+k^{\rho} \bar{\nabla}_{\rho} \ln F[\varphi]\right),
$$

where we have used the definition of $\bar{v}_{\mu}$ in terms of the slowly-varying configuration of the extra scalar field. After the transformation Eq. (5.6), the latter becomes

$$
\hat{k}^{\rho} \hat{\nabla}_{\rho} \ln \left(\mathcal{A}^{T} a\right)=-\frac{1}{2}\left(\hat{\nabla}_{\rho} \hat{k}^{\rho}+\hat{k}^{\rho} \hat{\nabla}_{\rho} \ln F\right),
$$

where $\hat{\nabla}_{\rho}$ is the covariant derivative respect $\hat{g}_{\mu \nu}$. At the zero order Eq. (5.36) is

$$
\begin{aligned}
\bar{k}^{\rho} \bar{\partial}_{\rho} \ln & \left(\overline{\mathcal{A}}^{T} \bar{a}\right)=\frac{\mathrm{d}}{\mathrm{d} \bar{\chi}} \ln \left(\overline{\mathcal{A}}^{T} \bar{a}\right)=-\frac{1}{2}\left(\bar{\partial}_{\rho} \bar{k}^{\rho}+\bar{k}^{\rho} \bar{\partial}_{\rho} \ln F_{0}\right)= \\
= & -\frac{1}{2}\left(\left[\bar{\partial}_{0} \bar{k}^{0}+\bar{\partial}_{i} \bar{k}^{i}\right]+\bar{k}^{\rho} \bar{\partial}_{\rho} \ln F_{0}\right)=-\frac{1}{2}\left(\bar{\partial}_{i} \hat{n}^{i}+\frac{\mathrm{d}}{\mathrm{d} \bar{\chi}} \ln F_{0}\right)=-\frac{1}{2}\left(\frac{\mathcal{P}_{i}^{i}}{\bar{\chi}}+\frac{\mathrm{d}}{\mathrm{d} \bar{\chi}} \ln F_{0}\right)= \\
= & -\frac{\mathrm{d}}{\mathrm{d} \bar{\chi}}\left(\ln \bar{\chi}+\ln \sqrt{F_{0}}\right)
\end{aligned}
$$

where we called $F_{0}=F\left[\varphi_{0}\right]$. We can rewrite the latter as

$$
\frac{\mathrm{d}}{\mathrm{d} \bar{\chi}} \ln \left(\overline{\mathcal{A}}^{T} \bar{a} \sqrt{F_{0}} \bar{\chi}\right)=0 \quad \longrightarrow \quad \overline{\mathcal{A}}^{T}\left(\bar{x}^{0}, \bar{\chi}\right)=\frac{\mathcal{Q}}{\bar{a}\left(\bar{x}^{0}\right) \bar{\chi} \sqrt{F_{0}}} .
$$

where $\mathcal{Q}$ is an integration constant determined by the local-wave zone solution and constant along the null geodesic. The GR equivalent of Eq. (5.37) is ${ }^{8}$

$$
\left(\overline{\mathcal{A}}^{T}\right)^{G R}\left(\bar{x}^{0}, \bar{\chi}\right)=\frac{\mathcal{Q}}{\bar{a}\left(\bar{x}^{0}\right) \bar{\chi}},
$$

where there is a factor $1 / \sqrt{F_{0}}$ of difference with respect to the result in modified gravity.

As in the case of the phase, we write the GW amplitude as a sum of the value it would have on the unperturbed background and a total linear correction

$$
\begin{aligned}
\ln \mathcal{A}^{T}\left(\bar{x}^{\mu}+\Delta x^{\mu}\right) & =\ln \overline{\mathcal{A}}^{T}(\bar{x}(\bar{\chi}))+\Delta \ln A^{T}(\bar{x}(\bar{\chi}))= \\
& =\ln \overline{\mathcal{A}}^{T}(\bar{x}(\bar{\chi}))+\delta \ln \mathcal{A}^{T}(\bar{x}(\bar{\chi}))+\Delta x^{\mu} \bar{\partial}_{\mu} \ln \overline{\mathcal{A}}^{T}(\bar{x}(\bar{\chi}))
\end{aligned}
$$

where $\delta \ln \mathcal{A}^{T}(\bar{x}(\bar{\chi}))=\ln \mathcal{A}^{T}(\bar{x}(\bar{\chi}))-\ln \overline{\mathcal{A}}^{T}(\bar{x}(\bar{\chi}))$ and plug this expansion into Eq. (5.36). The linearization of Eq. (5.36) is made of four different contributions, three of them do not

\footnotetext{
${ }^{8}$ To be precise, the integration constant $\mathcal{Q}$ could be different in modified gravity with respect to GR.
} 
depend explicitly on the extra scalar field and can be found also in the case of GR [107]. These are

$$
\begin{aligned}
& \text { 1) } \frac{\mathrm{d}}{\mathrm{d} \chi} \ln \left(\mathcal{A}^{T}\left(\bar{x}^{\mu}+\Delta x^{\mu}\right)\right)=\left(1-\frac{\mathrm{d} \delta \chi}{\mathrm{d} \bar{\chi}}\right)\left[\frac{\mathrm{d}}{\mathrm{d} \bar{\chi}} \ln \overline{\mathcal{A}}^{T}+\frac{\mathrm{d}}{\mathrm{d} \bar{\chi}} \delta \ln \mathcal{A}^{T}+\frac{\mathrm{d}}{\mathrm{d} \bar{\chi}}\left(\Delta x^{\mu} \bar{\partial}_{\mu} \ln \overline{\mathcal{A}}^{T}\right)\right]= \\
& =\frac{\mathrm{d} \ln \overline{\mathcal{A}}^{T}}{\mathrm{~d} \bar{\chi}}+\frac{\mathrm{d} \delta \ln \overline{\mathcal{A}}^{T}}{\mathrm{~d} \bar{\chi}}+\delta \chi \frac{\mathrm{d}^{2} \ln \overline{\mathcal{A}}^{T}}{\mathrm{~d} \bar{\chi}^{2}}+\delta k^{\mu} \bar{\partial}_{\mu} \ln \overline{\mathcal{A}}^{T}+\delta x^{\mu} \frac{\mathrm{d} \bar{\partial}_{\mu} \ln \overline{\mathcal{A}}^{T}}{\mathrm{~d} \bar{\chi}}, \\
& \text { 2) } \frac{\mathrm{d}}{\mathrm{d} \chi} \ln a\left(\bar{x}^{0}+\Delta x^{0}\right)=\left(1-\frac{\mathrm{d} \delta \chi}{\mathrm{d} \bar{\chi}}\right) \frac{\mathrm{d}}{\mathrm{d} \bar{\chi}} \ln \left[\bar{a}\left(1+\mathcal{H} \Delta x^{0}\right)\right]= \\
& =-\mathcal{H}\left(1-\frac{\mathrm{d} \delta \chi}{\mathrm{d} \bar{\chi}}\right)+\frac{\mathrm{d}}{\mathrm{d} \bar{\chi}}\left(\mathcal{H} \Delta x^{0}\right)=-\mathcal{H}\left[1-\delta k^{0}\right]-\mathcal{H}^{\prime}\left[\delta x^{0}-\delta \chi\right], \\
& \text { 3) }-\frac{1}{2} \hat{\nabla}_{\rho} \hat{k}^{\rho}=-\frac{1}{2}\left[\frac{\partial \bar{x}^{\mu}}{\partial x^{\rho}} \bar{\partial}_{\mu}\left(\bar{k}^{\rho}+\delta k^{\rho}\right)+\delta \hat{\Gamma}_{\rho \mu}^{\rho} \bar{k}^{\mu}\right]= \\
& =\frac{-1}{2}\left[\delta \hat{\Gamma}_{\rho \mu}^{\rho} \bar{k}^{\mu}+\frac{2}{\bar{\chi}}\left(1+\delta k_{\|}\right)+\frac{\mathrm{d} \delta k_{\|}}{\mathrm{d} \bar{\chi}}+\bar{\partial}_{0}\left(\delta k^{0}+\delta k_{\|}\right) \bar{\partial}_{i \perp} \delta k_{\perp}^{i}-\frac{2}{\bar{\chi}^{2}}\left(\delta \chi+\delta x_{\|}\right)-\frac{1}{\bar{\chi}} \bar{\partial}_{i \perp} \delta x_{\perp}^{i}\right],
\end{aligned}
$$

where $\delta \hat{\Gamma}_{\rho \mu}^{\rho}$ is the linear order connection coefficient associated to $\hat{g}_{\mu \nu}$. The new part with respect to GR is

$$
\begin{aligned}
4) & -\frac{1}{2} \frac{\mathrm{d}}{\mathrm{d} \chi} \ln F\left(\varphi_{0}+\Delta \varphi\right)=-\frac{1}{2}\left(1-\frac{\mathrm{d} \delta \chi}{\mathrm{d} \bar{\chi}}\right) \frac{\mathrm{d}}{\mathrm{d} \bar{\chi}} \ln \left[F_{0}\left(1+\frac{F_{\varphi 0}}{F_{0}} \Delta \varphi\right)\right]= \\
= & -\frac{1}{2}\left[\left(\frac{F_{\varphi 0}}{F_{0}}+\Delta \varphi\left(\frac{F_{\varphi \varphi 0}}{F_{0}}-\frac{F_{\varphi 0}^{2}}{F_{0}^{2}}\right)\right) \frac{\mathrm{d} \varphi_{0}}{\mathrm{~d} \bar{\chi}}+\frac{F_{\varphi 0}}{F_{0}}\left(\frac{\mathrm{d} \delta \varphi}{d \bar{\chi}}+\delta \chi \frac{\mathrm{d}^{2} \varphi_{0}}{d \bar{\chi}^{2}}+\delta k^{0} \varphi_{0}^{\prime}+\delta x^{0} \frac{\mathrm{d} \varphi_{0}^{\prime}}{d \bar{\chi}}\right)\right],
\end{aligned}
$$

were we used the short notations $\left.(\mathrm{d} F / \mathrm{d} \varphi)\right|_{\varphi_{0}}=F_{\varphi 0},\left.\left(\mathrm{~d}^{2} F / \mathrm{d} \varphi^{2}\right)\right|_{\varphi_{0}}=F_{\varphi \varphi 0}, \varphi_{0}^{\prime}=\bar{\partial}_{0} \varphi_{0}$ and $\Delta \varphi=\delta \varphi+\Delta x^{\mu} \bar{\partial}_{\mu} \varphi_{0}$. Combining together the four contributions yields

$$
\frac{\mathrm{d}}{\mathrm{d} \bar{\chi}} \delta \ln \left(\mathcal{A}^{T}\right)(\bar{\chi})=-\frac{1}{2}\left[\delta \hat{\Gamma}_{\rho \mu}^{\rho} \bar{k}^{\mu}+\frac{\mathrm{d} \delta k_{\|}}{\mathrm{d} \bar{\chi}}+\bar{\partial}_{0}\left(\delta k^{0}+\delta k_{\|}\right)-2 \frac{\mathrm{d} \kappa}{\mathrm{d} \bar{\chi}}+\frac{\mathrm{d}}{d \bar{\chi}}\left(\frac{F_{\varphi 0}}{F_{0}} \delta \varphi\right)\right]
$$

where $\kappa$ is the weak lensing convergence term defined as

$$
\kappa=-\frac{1}{2} \bar{\partial}_{i \perp} \Delta x_{\perp}^{i}
$$

Eq. (5.40) can be integrated in order to obtain $\delta \ln \mathcal{A}^{T}(\bar{\chi})$. The full correction to $\mathcal{A}^{T}$, defined through Eq. (5.39), then reads

$$
\begin{aligned}
\Delta \ln \mathcal{A}^{T} & =\delta \ln \mathcal{A}^{T}(\bar{\chi})+\Delta x^{0} \bar{\partial}_{0} \ln \overline{\mathcal{A}}^{T}+\Delta x^{i} \bar{\partial}_{i} \ln \overline{\mathcal{A}}^{T}= \\
& =\delta \ln \mathcal{A}^{T}(\bar{\chi})-\Delta \ln a\left(1+\frac{F_{\varphi 0} \varphi_{0}^{\prime}}{2 F_{0} \mathcal{H}}-\frac{1}{\bar{\chi} \mathcal{H}}\right)+\frac{T}{\bar{\chi}},
\end{aligned}
$$

where $T$ is given in Eq. (5.33). Besides contributions depending on cosmological fluctuations, we find an explicit additional term due to modified gravity. 


\subsection{Perturbations in the Poisson gauge}

The expressions obtained so far never actually used the explicit form of the metric Eq. (5.4), therefore they are valid for cosmological perturbations in any gauge. However, by using the Poisson gauge we can rewrite Eq. (5.40) in a more physically transparent and understandable way. As far as the phase is concerned, its corrections will not change in this particular gauge, hence we focus on the GW amplitude only.

The corrections $\delta k^{\mu}$ are related to the two scalar gravitational potentials $\phi$ and $\psi$ via the geodesic equation satisfied by the wave-vector. At first order it is

$$
\frac{\mathrm{d}}{\mathrm{d} \bar{\chi}} \delta k^{\mu}(\bar{\chi})+\delta \hat{\Gamma}_{\alpha \beta}^{\mu} \bar{k}^{\alpha}(\bar{\chi}) \bar{k}^{\beta}(\bar{\chi})=0,
$$

where $\delta \hat{\Gamma}_{\alpha \beta}^{\mu}$ are given by

$$
\begin{array}{ccc}
\delta \hat{\Gamma}_{00}^{0}=\phi^{\prime}, & \delta \hat{\Gamma}_{00}^{i}=\bar{\partial}^{i} \phi, & \delta \hat{\Gamma}_{i 0}^{0}=\bar{\partial}_{i} \phi \\
\delta \hat{\Gamma}_{i j}^{0}=-\delta_{i j} \psi^{\prime}, \delta \hat{\Gamma}_{j 0}^{i}=-\delta_{j}^{i} \psi^{\prime}, \delta \hat{\Gamma}_{j k}^{i}=-\delta_{k}^{i} \bar{\partial}_{j} \psi-\delta_{j}^{i} \bar{\partial}_{k} \psi+\delta_{j k} \bar{\partial}^{i} \psi
\end{array}
$$

We can then derive the set of equations

$$
\frac{\mathrm{d}}{\mathrm{d} \bar{\chi}}(\delta \nu-2 \phi)=\phi^{\prime}+\psi^{\prime}, \quad \frac{\mathrm{d}}{\mathrm{d} \bar{\chi}}\left(\delta n^{i}-2 \hat{n}^{i} \psi\right)=-\bar{\partial}^{i}(\phi+\psi) .
$$

Moreover, in the observer frame

$$
E_{\hat{0} \mu} \hat{k}^{\mu}=-(1+\phi)(-1+\delta \nu)+v_{i}\left(\hat{n}^{i}+\delta n^{i}\right)=1-\delta \nu+\phi+v_{\|},
$$

therefore the condition $\left.\left(E_{\hat{0} \mu} \hat{k}^{\mu}\right)\right|_{o}=1$ sets

$$
\delta \nu_{o}=\phi_{o}+v_{\| o}
$$

which we need as initial condition in order to integrate Eqs. (5.44). The initial condition for the spatial part of the wave vector is

$$
\delta n_{o}^{\hat{a}}=-v_{o}^{\hat{a}}+\hat{n}^{\hat{a}} \psi_{o} \quad \rightarrow \quad \delta n_{o}^{i}=-v_{o}^{i}+\hat{n}^{i} \psi_{o} .
$$

Using Eqs. (5.46)-(5.47), Eq. (5.44) can be integrated

$$
\begin{aligned}
& \delta k^{0}=\delta \nu=2 \phi-\left(\phi_{o}-v_{\| o}\right)+\int_{0}^{\bar{\chi}} d \tilde{\chi}\left(\phi^{\prime}+\psi^{\prime}\right), \\
& \delta k^{i}=\delta n^{i}=-v_{o}^{i}-\hat{n}^{i} \psi_{o}+2 \hat{n}^{i} \psi-\int_{0}^{\bar{\chi}} d \tilde{\chi} \bar{\partial}^{i}(\phi+\psi)=\hat{n}^{i} \delta n_{\|}+\delta n_{\perp}^{i},
\end{aligned}
$$

and

$$
\delta n_{\|}=\phi_{o}-v_{\| o}-\phi+\psi+2 I, \quad \delta n_{\perp}^{i}=-v_{\perp o}^{i}+2 S_{\perp}^{i},
$$

where we defined

$$
I=-\frac{1}{2} \int_{0}^{\bar{\chi}} d \tilde{\chi}\left(\phi^{\prime}+\psi^{\prime}\right), \quad S_{\perp}^{i}=-\frac{1}{2} \int_{0}^{\bar{\chi}} d \tilde{\chi} \bar{\partial}_{\perp}^{i}(\phi+\psi) .
$$

We recognize in $I$ the integrated Sachs-Wolfe effect. Furthermore we have

$$
\bar{\partial}_{0}\left(\delta k^{0}+\delta k_{\|}\right)=\phi^{\prime}+\psi^{\prime}, \quad \delta \Gamma_{\rho \mu}^{\rho} \bar{k}^{\mu}=-\phi^{\prime}+3 \psi^{\prime}+\hat{n}^{i} \bar{\partial}_{i} \phi-3 \hat{n}^{i} \bar{\partial}_{i} \psi=\frac{\mathrm{d} \phi}{\mathrm{d} \bar{\chi}}-3 \frac{\mathrm{d} \psi}{\mathrm{d} \bar{\chi}} .
$$


With this expressions we can find $\delta \ln \mathcal{A}^{T}$ in the Poisson gauge by integrating Eq. (5.40)

$$
\delta \ln \mathcal{A}^{T}=\delta \ln \mathcal{A}_{o}+\kappa+\psi-\psi_{o}-\frac{1}{2}\left[\left(\frac{F_{\varphi 0}}{F_{0}} \delta \varphi\right)(\bar{\chi})-\left(\frac{F_{\varphi 0}}{F_{0}} \delta \varphi\right)_{o}\right],
$$

where we used $\kappa_{o}=I_{o}=0$ as it should be as these two are integrated effects. In order to obtain $\Delta \ln \mathcal{A}^{T}$ as in Eq. (5.41), we need also $\Delta \ln a$ and $T$, respectively they are

$$
\begin{aligned}
& \Delta \ln a=\mathcal{H} \Delta x^{0}=E_{\hat{0} \mu}^{(1)} \bar{k}^{\mu}+E_{\hat{0} \mu}^{(0)} \delta k^{\mu}=\left(\phi_{o}-v_{\| o}\right)-\left(\phi-v_{\|}\right)+2 I, \\
& T=-\int_{0}^{\bar{\chi}} d \tilde{\chi}(\phi+\psi) .
\end{aligned}
$$

In this way, the total correction to $\mathcal{A}^{T}$ is ${ }^{9}$

$$
\begin{aligned}
\Delta \ln \mathcal{A}^{T}(\bar{\chi})=\kappa+ & \psi-\frac{1}{2}\left(\frac{F_{\varphi 0}}{F_{0}} \delta \varphi\right)(\bar{\chi})-\frac{1}{\bar{\chi}} \int_{0}^{\bar{\chi}} d \tilde{\chi}(\phi+\psi) \\
& -\left(\phi_{o}-v_{\| o}-\phi+v_{\|}+2 I\right)\left(1+\frac{F_{\varphi 0} \varphi_{0}^{\prime}}{2 F_{0} \mathcal{H}}-\frac{1}{\bar{\chi} \mathcal{H}}\right) .
\end{aligned}
$$

The weak-lensing convergence term was $\kappa$ can be also written in the more familiar way

$$
\kappa=-\frac{1}{2} \bar{\partial}_{i \perp} \Delta x_{\perp}^{i}=\frac{1}{2} \int_{0}^{\bar{\chi}} d \tilde{\chi} \frac{(\bar{\chi}-\tilde{\chi})}{\tilde{\chi} \bar{\chi}} \triangle_{\Omega}(\phi+\psi),
$$

where $\triangle_{\Omega}=\tilde{\chi}^{2} \bar{\nabla}_{\perp}^{2}$, using

$$
\Delta x_{\perp}^{i}=\delta x_{\perp}^{i}=\int_{0}^{\bar{\chi}} d \chi \delta n_{\perp}^{i}=-v_{\perp o}^{i} \bar{\chi}-\int_{0}^{\bar{\chi}} d \tilde{\chi}(\bar{\chi}-\tilde{\chi}) \tilde{\partial}_{\perp}^{i}(\phi+\psi) .
$$

\subsection{The luminosity distance}

We can use the above results to write the full form of the gravitational wave in the geometrical optics limit

$$
\bar{h}_{\mu \nu}^{T T}=\mathcal{A}^{T} e_{\mu \nu}^{T T} e^{i \theta / \epsilon}=\overline{\mathcal{A}}^{T}\left(\bar{x}^{0}, \bar{\chi}\right)\left(1+\Delta \ln \mathcal{A}^{T}\right) e_{\mu \nu}^{T T} e^{i(\bar{\theta}(\bar{\chi})+\Delta \theta) / \epsilon} .
$$

The amplitude at emission is given by

$$
\mathcal{A}^{T}\left(\bar{\eta}_{e}, \bar{x}_{e}^{i}\right)=\overline{\mathcal{A}}^{T}\left(1+\Delta \ln \mathcal{A}^{T}\right)=\frac{\mathcal{Q}(1+z)^{2}}{\bar{D}_{L}^{G R} \sqrt{F_{0}}}\left(1+\Delta \ln \left(\mathcal{A}^{T}\right)\right),
$$

where we used Eq. (5.37) and $\bar{a}\left(\eta_{e}\right)=(1+z)^{-1} \cdot \bar{D}_{L}^{G R}=(1+z) \bar{\chi}$ is the observed average luminosity distance taken over all the sources with the same observed redshift in GR. We can define the following quantities

$$
\begin{aligned}
\bar{D}_{L}^{g w} & =\bar{D}_{L}^{G R} \sqrt{F_{0}}, \\
D_{L}^{g w} & =\frac{\bar{D}_{L}^{G R} \sqrt{F_{0}}}{\left(1+\Delta \ln \mathcal{A}^{T}\right)}=\bar{D}_{L}^{g w}\left(1-\Delta \ln \mathcal{A}^{T}\right),
\end{aligned}
$$

\footnotetext{
${ }^{9}$ We have also used that by construction $\delta \ln \mathcal{A}_{o}^{T}=\psi_{o}+\frac{1}{2}\left(\frac{F_{\varphi 0}}{F_{0}} \delta \varphi\right)_{o}$
} 
therefore, the relative correction to the gravitational luminosity distance is

$$
\frac{\Delta D_{L}^{g w}}{\bar{D}_{L}^{g w}}=\frac{D_{L}^{g w}-\bar{D}_{L}^{g w}}{\bar{D}_{L}^{g w}}=-\Delta \ln \mathcal{A}^{T}(\bar{\chi}) .
$$

The gravitational wave at the detector is also red-shifted. We can use Eq. (5.63) and Eq. (5.56) to write

$$
\begin{aligned}
\frac{\Delta D_{L}^{g w}}{\bar{D}_{L}^{g w}} & =\left(1-\frac{1}{\mathcal{H} \bar{\chi}}+\frac{F_{\varphi 0} \varphi_{0}^{\prime}}{2 F_{0} \mathcal{H}}\right) v_{\|}-\frac{1}{2} \int_{0}^{\bar{\chi}} d \tilde{\chi} \frac{(\bar{\chi}-\tilde{\chi})}{\tilde{\chi} \bar{\chi}} \Delta_{\Omega}(\phi+\psi) \\
& +\phi\left(\frac{1}{\bar{\chi} \mathcal{H}}-\frac{F_{\varphi 0} \varphi_{0}^{\prime}}{2 F_{0} \mathcal{H}}\right)-\left(1+\frac{F_{\varphi 0} \varphi_{0}^{\prime}}{2 F_{0} \mathcal{H}}-\frac{1}{\bar{\chi} \mathcal{H}}\right) \int_{0}^{\bar{\chi}} d \tilde{\chi}\left(\phi^{\prime}+\psi^{\prime}\right) \\
& -\left(\phi+\psi-\frac{F_{\varphi 0}}{2 F_{0}} \delta \varphi(\bar{\chi})\right)+\frac{1}{\bar{\chi}} \int_{0}^{\bar{\chi}} d \tilde{\chi}(\phi+\psi),
\end{aligned}
$$

where we dropped the unobservable constant contribution evaluated at the observer position. If we choose $F=$ const, we recover the correct limit to GR, which can be found in [107]; on the other hand, Eq. (5.64) shows how modified gravity alters the GW luminosity distance.

In fact, in Eq. (5.64) it is possible to distinguish six different contributions:

- a peculiar velocity contribution, explicitly modified by dynamical dark energy;

- a weak-lensing contribution, not explicitly altered;

- a Sachs-Wolfe effect, explicitly modified;

- an integrated Sachs-Wolfe effect, explicitly modified;

- volume effects, explicitly modified;

- a contribution from Shapiro time delay, not explicitly altered dynamical dark energy.

We stress that, on top of the explicit modifications in Eq. (5.64), due to the presence of the extra degree of freedom, also the dynamics of $\phi$ and $\psi$ is also potentially different with respect to GR.

\subsection{The polarization tensor}

We turn now to the evolution equation of the polarization tensor $\mathbf{e}_{\mu \nu}$. In order to avoid cumbersome computations we will focus on the subclass in which $G_{X}=0$, which is compatible with the gauge conditions given by Eqs. (3.7)-(3.9). Only in this sub-case we can invoke screening mechanisms to fix $\mathcal{A}^{S}=0$ so that Eq. (5.2) becomes

$$
k^{\rho} \bar{\nabla}_{\rho} \mathbf{e}_{\mu \nu}=0
$$

After the conformal transformation, Eq. (5.6), Eq. (5.65) becomes

$$
\hat{k}^{\rho} \hat{\nabla}_{\rho}\left(\mathbf{e}_{\mu \nu} a^{-2}\right)=0
$$

In the transformed spacetime the polarization $\mathbf{e}_{\mu \nu}$ has to satisfy the constraint

$$
\hat{k}^{\mu} \mathbf{e}_{\mu \nu}=0,
$$


coming from the harmonic gauge. As we did for the phase and the amplitude of the GW, we expand the polarization tensor as

$$
\begin{aligned}
\mathbf{e}_{\mu \nu}\left(x^{\mu}(\chi)\right)=\mathbf{e}_{\mu \nu}\left(\bar{x}^{\mu}+\Delta x^{\mu}\right) & =\overline{\mathbf{e}}_{\mu \nu}\left(\bar{x}^{\mu}(\bar{\chi})\right)+\Delta \mathbf{e}_{\mu \nu}\left(\bar{x}^{\mu}(\bar{\chi})\right)= \\
& =\overline{\mathbf{e}}_{\mu \nu}\left(\bar{x}^{\mu}(\bar{\chi})\right)+\delta \mathbf{e}_{\mu \nu}\left(\bar{x}^{\mu}(\bar{\chi})\right)+\Delta x^{\rho} \bar{\partial}_{\rho} \overline{\mathbf{e}}_{\mu \nu}\left(\bar{x}^{\mu}(\bar{\chi})\right)
\end{aligned}
$$

where $\overline{\mathbf{e}}_{\mu \nu}$ is the value that $\mathbf{e}_{\mu \nu}$ would have on a unperturbed space-time, while $\Delta \mathbf{e}_{\mu \nu}=$ $\delta \mathbf{e}_{\mu \nu}+\Delta x^{\rho} \bar{\partial}_{\rho} \overline{\mathbf{e}}_{\mu \nu}$ is the linear correction due to the presene of inhomogeneities. As in the previous cases, we defined $\delta \mathbf{e}_{\mu \nu}(\bar{x}(\bar{\chi})):=\mathbf{e}_{\mu \nu}(\bar{x}(\bar{\chi}))-\overline{\mathbf{e}}_{\mu \nu}(\bar{x}(\bar{\chi}))$. The expansion of Eq. (5.66) at linear order is

$$
\begin{aligned}
\hat{k}^{\rho} \hat{\nabla}_{\rho}\left(\mathbf{e}_{\mu \nu} a^{-2}\right) & =\frac{\mathrm{d}}{\mathrm{d} \chi}\left(\mathbf{e}_{\mu \nu} a^{-2}\right)=\left(1-\frac{\mathrm{d} \delta \chi}{\mathrm{d} \chi}\right) \frac{\mathrm{d}}{\mathrm{d} \bar{\chi}}\left(\left(\overline{\mathbf{e}}_{\mu \nu}+\Delta \mathbf{e}_{\mu \nu}\right) \bar{a}^{-2}(1-2 \Delta \ln a)\right)= \\
& =\frac{\mathrm{d}\left(\overline{\mathbf{e}}_{\mu \nu} \bar{a}^{-2}\right)}{\mathrm{d} \bar{\chi}}+\frac{\mathrm{d}\left(\delta \mathbf{e}_{\mu \nu} \bar{a}^{-2}\right)}{\mathrm{d} \bar{\chi}}+\frac{\mathrm{d}\left(\delta x^{\rho} \bar{\partial}_{\rho}\left(\overline{\mathbf{e}}_{\mu \nu} \bar{a}^{-2}\right)\right)}{\mathrm{d} \bar{\chi}}+\delta \chi \frac{\mathrm{d}^{2}\left(\overline{\mathbf{e}}_{\mu \nu} \bar{a}^{-2}\right)}{\mathrm{d} \bar{\chi}^{2}},
\end{aligned}
$$

where we have considered that in $\hat{\nabla}_{\rho}$ the connection coefficients are of linear order in the metric perturbation as $\hat{\nabla}_{\rho}$ is the covariant derivative associated to the metric $\hat{g}_{\mu \nu}=\eta_{\mu \nu}+\delta \hat{g}_{\mu \nu}$. Therefore in Eq. (5.69), $\Delta x^{\rho} \hat{\nabla}_{\rho}\left(\overline{\mathbf{e}}_{\mu \nu} \bar{a}^{-2}\right)=\Delta x^{\rho} \bar{\partial}_{\rho}\left(\overline{\mathbf{e}}_{\mu \nu} \bar{a}^{-2}\right)$ at the order we are restricting ourselves to, as $\Delta x^{\rho}$ is already a first order quantity. Consequently, the zeroth order of Eq. (5.66) is

$$
\frac{\mathrm{d}\left(\overline{\mathbf{e}}_{\mu \nu} \bar{a}^{-2}\right)}{\mathrm{d} \bar{\chi}}=0 \quad \rightarrow \quad \overline{\mathbf{e}}_{\mu \nu}=\bar{a}^{2} \mathcal{Q}_{\mu \nu}
$$

where $\mathcal{Q}_{\mu \nu}$ is a constant, transverse tensor. The linear order of Eq. (5.66)'s expansion is

$$
\frac{\mathrm{d}\left(\delta \mathbf{e}_{\mu \nu} \bar{a}^{-2}\right)}{\mathrm{d} \bar{\chi}}+\frac{\mathrm{d}\left(\delta x^{\rho} \bar{\partial}_{\rho}\left(\overline{\mathbf{e}}_{\mu \nu} \bar{a}^{-2}\right)\right)}{\mathrm{d} \bar{\chi}}+\delta \chi \frac{\mathrm{d}^{2}\left(\overline{\mathbf{e}}_{\mu \nu} \bar{a}^{-2}\right)}{\mathrm{d} \bar{\chi}^{2}}=0
$$

The latter can be simplified using the result of Eq. (5.70)

$$
\frac{\mathrm{d}\left(\delta \mathbf{e}_{\mu \nu} \bar{a}^{-2}\right)}{\mathrm{d} \bar{\chi}}=0 .
$$

This equation allows us to obtain $\delta \mathbf{e}_{\mu \nu} \bar{a}^{-2}$ by integration. Then, the full correction to the GW polarization, i.e. $\Delta \mathbf{e}_{\mu \nu}(\bar{\chi})$ is given by

$$
\Delta \mathbf{e}_{\mu \nu}=\delta \mathbf{e}_{\mu \nu}+2 \mathcal{H} \Delta x^{0} \overline{\mathbf{e}}_{\mu \nu} .
$$

where we used Eq. (5.70) and that Christoffel symbols are null at zero order. We point out that $\mathcal{H} \Delta x^{0}$ contains, as given in Eq. (5.54), effects such as the ISW and doppler. We plan to further study the behavior of the polarization tensor in the case of braiding, where it is not parallel-transported in future works.

\section{Conclusions}

The detection of gravitational waves (GWs) propagating over cosmological distances will offer new opportunities for probing cosmological structures in our Universe, as well as for testing possible departures from GR. In this work we established tools for studying GW 
propagation in scalar-tensor theories of gravity, adopting a geometrical ansatz, and focusing on high frequency spin- 2 and spin-0 modes travelling at the light speed on slowly-varying backgrounds, gauging away all the spurious modes. Our approach can apply to scenarios with non-minimal couplings between scalar and tensor degrees of freedom: we took particular care in discussing and motivating our gauge choices- see Eqs. (2.45)-(2.47) - and to develop general arguments that do not rely on the particular choice of the scalar-tensor theory.

We determined the general structure of the evolution equations for the GW amplitude and polarization tensor. We found that they can be different with respect to GR - see in particular Eqs. (3.14)-(3.15), and Eqs. (3.32)-(3.31). In theories which preserve the graviton number, the equation for the amplitude of GWs can differ from GR if the effective Planck scale varies over the GW geodesics. Interestingly, we also find that the GW polarization tensor can fail to be parallel transported along the GW geodesics, and discussed physical interpretations of our results. We applied our general formulas to a representative example of scalar-tensor scenarios considering, for the first time in the literature, scalar waves, SWs, and tensor waves, GWs, both travelling with unit speed. In this context, Eqs. (4.14)-(4.16) represent the most general results of this work. They show that the GW propagation depends both on background quantities and the assumed scalar-tensor theory, that the amplitude $\mathcal{A}^{T}$ of the tensor mode does not depend on its scalar counterpart $\mathcal{A}^{S}$, and, that the tensor and scalar modes of polarization are generally coupled to each other. See also [125] for a recent paper focussing on the propagation of tensor modes.

An important advantage of our approach is that it can be applied to study the propagation of GWs on perturbed cosmological space-times, provided that the wavelength of cosmological fluctuations is well larger than the GW wavelength. In fact, under the simplified assumption that $\mathcal{A}^{S}$ is suppressed at the GW emission and absence of braiding, we derived the general evolution equations controlling GW propagation in such a set-up, distinguishing and identifying the distinct contributions associated with cosmological fluctuations, and with scalar-tensor effects.

More precisely, at first order in perturbation theory, we determined several corrections to standard formulas for the GW luminosity distance - Eq. (5.64). We found that the contribution to the GW luminosity distance from the peculiar velocity, Sachs-Wolfe, integrated Sachs-Wolfe and volume effects are explicitly modified in the presence of scalar-tensor interactions. On the other hand, weak-lensing and Shapiro time delay are only implicitly altered, via the modified dynamics of $\phi$ and $\psi$, due to the presence of the extra degree of freedom.

Finally, under the assumption $G_{X}=0$ and $\mathcal{A}^{S}=0$, we obtained the evolution of the transverse polarization tensor, Eq. (5.72), in the linear regime. We argued that only in this specific sub-case the polarization is parallel transported along the geodesics of the GW. We plan to investigate the dynamics of the polarization in presence of braiding, where it is not parallel transported, in a future work.

Starting from the general results presented here, much work is left for the future. It would be interesting to apply our method to more general scalar-tensor theories than the representative examples discussed here, or to extend our approach to modified gravity scenarios with multiple fields. It would be interesting to study in more detail the physical consequences of our findings for GWs propagating over cosmological distances, and analyze in detail degeneracies between modified gravity and effects of non-linearities as well as understanding the effect of the the self-induced GW energy momentum tensor in theories alternative to GR. We hope to return soon to these topics. 


\section{Acknowledgments}

It is a pleasure to thank Nicola Bartolo, Giulia Cusin, Charles Dalang, Pierre Fleury, Alexander Ganz, Luigi Guzzo, Lucas Lombriser, Angelo Ricciardone and Nicolás Yunes for useful discussions. C.C. warmly thanks Andrew J. S. Hamilton for very useful clarifications. The work of G.T. is partially supported by STFC grant ST/P00055X/1. D.B. and S.M. acknowledge partial financial support by ASI Grant No. 2016-24-H.0.

\section{A Conditions to ensure a unit speed for scalar fluctuations}

In this Appendix we briefly discuss some constraints that the compatibility condition in Eq. (2.43) imposes on the scalar-tensor systems and the background solutions under consideration. For definiteness, we consider the scalar-tensor theory

$$
\mathcal{L}^{\text {tot }}=F(\varphi) R+G(\varphi, X) \square \varphi+K(\varphi, X),
$$

and derive a constraint on $K$ and $G$ that will guarantee the compatibility condition to be satisfied. In order to do se we linearize to first order in $\varphi_{r}$ and $\hat{h}_{\mu \nu}$ the scalar field equation, $\partial \mathcal{L}^{t o t} / \partial \varphi=0$

$$
\begin{aligned}
& \left(\bar{F}_{\varphi} \bar{g}^{\mu \nu}-\bar{G}_{X} \bar{\varphi}^{\mu} \bar{\varphi}^{\nu}\right)\left[R_{\mu \nu}^{(1)}\right]_{\epsilon^{-2}}+ \\
& +\frac{\left(\varphi_{\mu \nu}\right)_{\epsilon^{-1}}^{(1)}}{\bar{F}}\left[\bar{g}^{\mu \nu}\left(3 \bar{F}_{\varphi}^{2}+2 \bar{X} \bar{F}_{\varphi} \bar{G}_{X}+\bar{X}^{2} \bar{G}_{X}^{2}+\bar{F} A\right)-\frac{\bar{F} \bar{G}_{X X}}{2}\left(\bar{X}^{\mu} \bar{\varphi}^{\nu}+\bar{X}^{\nu} \bar{\varphi}^{\mu}\right)-\right. \\
& \left.\quad-\bar{\varphi}^{\mu} \bar{\varphi}^{\nu}\left(2 \bar{F}_{\varphi} \bar{G}_{X}+\bar{X} \bar{G}_{X}^{2}+\bar{F} B\right)-2 \bar{F} \bar{G}_{X} \bar{\varphi}^{\mu \nu}\right]+\mathcal{O}\left(\epsilon^{0}\right)=0
\end{aligned}
$$

with $A=\left(\bar{\varphi}^{\mu} \bar{\nabla}_{\mu} \bar{G}_{X}+\bar{K}_{X}+2 \bar{G}_{\varphi}+2 \bar{G}_{X} \bar{\square} \bar{\varphi}\right), B=\left(\bar{K}_{X X}+2 \bar{G}_{X \varphi}+\bar{G}_{X X} \bar{\square} \bar{\varphi}\right)$ and $\varphi_{\nu}=\bar{\nabla}_{\nu} \varphi$, $\varphi_{\mu \nu}=\bar{\nabla}_{\nu} \bar{\nabla}_{\mu} \varphi$. At leading order, $\mathcal{O}\left(\epsilon^{-2}\right)$, the equation above gives

$$
\left[R_{\mu \nu}^{(1)}\right]_{1 / \epsilon^{2}}=0
$$

which is consistent with the gravitational field equations. At next-to-leading order, $\mathcal{O}\left(\epsilon^{-1}\right)$,

$$
\begin{aligned}
\frac{\left(\varphi_{\mu \nu}\right)_{\epsilon^{-1}}^{(1)}}{\bar{F}}\left[\overline { g } ^ { \mu \nu } \left(3 \bar{F}_{\varphi}^{2}+2 \bar{X} \bar{F}_{\varphi} \bar{G}_{X}\right.\right. & \left.+\bar{X}^{2} \bar{G}_{X}^{2}+\bar{F} A\right)-\frac{\bar{F} \bar{G}_{X X}}{2}\left(\bar{X}^{\mu} \bar{\varphi}^{\nu}+\bar{X}^{\nu} \bar{\varphi}^{\mu}\right)- \\
& \left.-\bar{\varphi}^{\mu} \bar{\varphi}^{\nu}\left(2 \bar{F}_{\varphi} \bar{G}_{X}+\bar{X} \bar{G}_{X}^{2}+\bar{F} B\right)-2 \bar{F} \bar{G}_{X} \bar{\varphi}^{\mu \nu}\right]=0,
\end{aligned}
$$

which, in the case $\bar{K}_{X X}=0$ and $G_{X}=0$ reduces to

$$
\frac{\left(3 \bar{F}_{\varphi}^{2}+\bar{F} \bar{K}_{X}+2 \bar{F} \bar{G}_{\varphi}\right)}{\bar{F}}(\square \varphi)_{1 / \epsilon^{1}}^{(1)}=0 .
$$

We can safely assume $\bar{F} \bar{K}_{X} \neq 0$, otherwise neither $g_{\mu \nu}$ nor $\varphi$ have a kinetic term in the Lagrangian to begin with, so that

$$
(\square \varphi)_{1 / \epsilon^{1}}^{(1)}=0,
$$


so that the compatibility of the gauge choices is guaranteed by the equation of motion. This result can be generalized to a group of theories larger than those satisfying $G_{X}=0$ by choosing $G(\varphi, X)$ such that

$$
\frac{\bar{F} \bar{G}_{X X}}{2}\left(\bar{X}^{\mu} \bar{\varphi}^{\nu}+\bar{X}^{\nu} \bar{\varphi}^{\mu}\right)+\bar{\varphi}^{\mu} \bar{\varphi}^{\nu}\left(2 \bar{F}_{\varphi} \bar{G}_{X}+\bar{X} \bar{G}_{X}^{2}+\bar{F} B\right)+2 \bar{F} \bar{G}_{X} \bar{\varphi}^{\mu \nu}=0 .
$$

It is important to point out that this condition is quite restrictive and not only sets constraints on the structure of the functions $F, G$, and $B$, but also on the slowly-varying background profile of $\bar{\varphi}$. Depending on the details of the system, one would need to investigate whether this condition can be satisfied and is compatible with the scalar equations of motion, which can also include additional contributions from couplings of the slowly-varying scalar with additional degrees of freedom. A complete analysis of this issue goes beyond the scope of this work.

\section{B Defining spin-2 and spin-0 polarization tensors using tetrads}

This Appendix aims to explain the method we use in the main text for disentangling the evolution equations for the amplitude of tensor and scalar modes.

We introduce null tetrads as in $[53,115]$

$$
z_{a}^{\mu}=\left(k^{\mu}, n^{\mu}, m^{\mu}, \bar{m}^{\mu}\right) ;
$$

$k^{\mu}$ and $n^{\mu}$ are real, $m^{\mu}$ complex, and $\bar{m}^{\mu}$ its complex conjugate. These vectors are null with respect to background metric $\bar{g}_{\mu \nu}$, that is (raising/lowering with metric $\bar{g}_{\mu \nu}$ ):

$$
0=k_{\mu} k^{\mu}=m_{\mu} m^{\mu}=\bar{m}_{\mu} \bar{m}^{\mu}=n_{\mu} n^{\mu}
$$

The only other non-vanishing products are

$$
\bar{g}_{\mu \nu} m^{\mu} \bar{m}^{\nu}=1 \quad \text { and } \quad \bar{g}_{\mu \nu} k^{\mu} n^{\nu}=-1
$$

The vector $k^{\mu}$ is identified with the GW direction. We can decompose a rank 2 symmetric tensor on the null-tetrad basis as

$$
A_{\mu \nu}=\alpha_{A B} \Theta_{\mu \nu}^{A B}
$$

with

$$
\Theta_{\mu \nu}^{A B}=\frac{1}{2}\left(A_{\mu} B_{\nu}+A_{\nu} B_{\mu}\right) \quad \text { and } \quad A_{\mu}, B_{\mu}=\left(k_{\mu}, n_{\mu}, m_{\mu}, \bar{m}_{\mu}\right),
$$

which shows that $\mathcal{A}_{\mu \nu}$ has ten independent component as it should be for a rank 2 symmetric tensor. Using the orthogonality relations between the elements of $z_{a}^{\mu}$, it is possible to show that the objects $\Theta_{\mu \nu}^{A B}$ satisfy

$$
\Theta_{\mu \nu}^{m m} \Theta_{\bar{m} \bar{m}}^{\mu \nu}=\Theta_{\mu \nu}^{\bar{m} \bar{m}} \Theta_{m m}^{\mu \nu}=\Theta_{\mu \nu}^{n n} \Theta_{k k}^{\mu \nu}=1, \quad \Theta_{\mu \nu}^{n m} \Theta_{k \bar{m}}^{\mu \nu}=\Theta_{\mu \nu}^{n \bar{m}} \Theta_{k m}^{\mu \nu}=-\Theta_{\mu \nu}^{m \bar{m}} \Theta_{m \bar{m}}^{\mu \nu}=-\frac{1}{2},
$$

while the other products vanish. Since we are interested in the geometric-optics limits to study the propagation of GWs, we apply the null-tetrad formalism to $\mathcal{A}_{\mu \nu}$ which is defined as $\hat{h}_{\mu \nu}=\mathcal{A}_{\mu \nu} e^{i \theta / \epsilon}$ and satisfy the gauge conditions given in Eqs. (3.7), (3.8) and (3.9). In 
this case, the tensor $\mathcal{A}_{\mu \nu}$ represents a null-wave so the ten independent components have a clear physical meaning as they are related to the Newmann-Penrose scalars (see [117]). In particular

$$
\begin{aligned}
\Psi_{2} & =\frac{1}{12} \ddot{\mathcal{A}}_{k k} \rightarrow \text { Longitudinal scalar mode } \mathrm{s}=0, \in \mathbb{R} \\
\Phi_{22} & =\frac{1}{2} \ddot{\mathcal{A}}_{m \bar{m}} \rightarrow \text { Transverse scalar mode } \mathrm{s}=0, \in \mathbb{R} \\
\Psi_{3} & =\frac{1}{4} \ddot{\mathcal{A}}_{k m} \rightarrow \text { Vector, mixed scalar/longitudinal, } \mathrm{s}= \pm 1, \in \mathbb{C} \rightarrow \exists \bar{\Psi}_{3} \\
\Psi_{4} & =\frac{1}{2} \ddot{\mathcal{A}}_{m m} \rightarrow \text { Tensor, transverse and traceless }, \mathrm{s} \pm 2, \in \mathbb{C} \rightarrow \exists \bar{\Psi}_{4}
\end{aligned}
$$

while $\Theta_{\mu \nu}^{n n}, \Theta_{\mu \nu}^{n k}, \Theta_{\mu \nu}^{n m}$ and $\Theta_{\mu \nu}^{n \bar{m}}$ are not associated to any physical observable. In fact it is possible to show that the condition $k^{\mu} \mathcal{A}_{\mu \nu}=0$ implies $\alpha_{n n}=\alpha_{n k}=\alpha_{n m}=\alpha_{n \bar{m}}=0$ because the only non vanishing scalar product is $k^{\mu} n_{\mu}=-1$. So a null transverse wave actually has only six independent components

$$
\begin{aligned}
\mathcal{A}_{\mu \nu} & =\alpha_{k k} \Theta_{\mu \nu}^{k k}+\alpha_{m m} \Theta_{\mu \nu}^{m m}+\alpha_{\bar{m} \bar{m}} \Theta_{\mu \nu}^{\bar{m} \bar{m}}+\alpha_{k m} \Theta_{\mu \nu}^{k m}+\alpha_{k \bar{m}} \Theta_{\mu \nu}^{k \bar{m}}+\alpha_{m \bar{m}} \Theta_{\mu \nu}^{m \bar{m}}= \\
& =\alpha_{C D} \Theta_{\mu \nu}^{C D},
\end{aligned}
$$

with $C_{\mu}, D_{\mu}=\left(k_{\mu}, m_{\mu}, \bar{m}_{\mu}\right)$. In the main text we used $\mathbf{e}_{\mu \nu}^{+}, \mathbf{e}_{\mu \nu}^{\times}$and $\mathbf{e}_{\mu \nu}^{S}$ which are defined as

$$
\mathbf{e}_{\mu \nu}^{+}=\frac{1}{\sqrt{2}}\left(\Theta_{\mu \nu}^{m m}+\Theta_{\mu \nu}^{\bar{m} \bar{m}}\right), \quad \mathbf{e}_{\mu \nu}^{\times}=\frac{i}{\sqrt{2}}\left(\Theta_{\mu \nu}^{\bar{m} \bar{m}}-\Theta_{\mu \nu}^{m m}\right), \quad \mathbf{e}_{\mu \nu}^{S}=\sqrt{2} \Theta_{\mu \nu}^{m \bar{m}}
$$

and they are all normalized to 1 and they are orthogonal to each other. The amplitudes $\mathcal{A}^{+}$, $\mathcal{A}^{\times}$and $\mathcal{A}^{S}$ are related to $\alpha_{C D}$ as

$$
\mathcal{A}^{+}=\frac{1}{\sqrt{2}}\left(\alpha_{m m}+\alpha_{\bar{m} \bar{m}}\right), \quad \mathcal{A}^{\times}=\frac{i}{\sqrt{2}}\left(\alpha_{m m}-\alpha_{\bar{m} \bar{m}}\right), \quad \text { and } \quad \mathcal{A}^{S}=\frac{\alpha_{m \bar{m}}}{\sqrt{2}} .
$$

\section{B.1 Gauge conditions and null tetrads}

We can use the decomposition of $\mathcal{A}_{\mu \nu}$ on the null-tetrad basis to treat the vector $\Psi_{3}$ and scalar-longitudinal mode $\Psi_{2}$, namely $\alpha_{k m}, \alpha_{k \bar{m}}$ and $\alpha_{k k}$. In fact, the null tetrad basis allows us to nicely distinguish the non-physical modes that we would like to gauge away. In Section 2.5 of the main text we claimed that we are entitle to make a last gauge transformation using the vector field $\sigma_{\mu}$, which has to satisfy $\square \sigma_{\mu}=\bar{v}^{\mu} \sigma_{\mu}=0$, to fix 3 components of the metric perturbation. We use this last gauge freedom to choose a particular form of the vector $\mathcal{C}_{\mu}=\bar{v}^{\mu} \mathcal{A}_{\mu \nu}$ such that $\alpha_{k m}, \alpha_{k \bar{m}}, \alpha_{k k}$ are fixed. From Eq. (B.11) it follows

$$
\begin{aligned}
\mathcal{C}_{\mu}= & \bar{v}^{\mu} \mathcal{A}_{\mu \nu}=\bar{v}^{\nu}\left(\alpha_{C D} \Theta_{\mu \nu}^{C D}\right)= \\
=\frac{1}{2}[ & k_{\mu}\left(2 \alpha_{k k}(\bar{v} \cdot k)+\alpha_{k m}(\bar{v} \cdot m)+\alpha_{k \bar{m}}(\bar{v} \cdot \bar{m})\right)+ \\
& +m_{\mu}\left(2 \alpha_{m m}(\bar{v} \cdot m)+\alpha_{k m}(\bar{v} \cdot k)+\alpha_{m \bar{m}}(\bar{v} \cdot \bar{m})\right)+ \\
& \left.+\bar{m}_{\mu}\left(2 \alpha_{\bar{m} \bar{m}}(\bar{v} \cdot \bar{m})+\alpha_{k \bar{m}}(\bar{v} \cdot k)+\alpha_{m \bar{m}}(\bar{v} \cdot m)\right)\right] .
\end{aligned}
$$


We can decompose $\mathcal{C}_{\mu}$ on the null tetrad basis as

$$
\mathcal{C}_{\mu}=\mathcal{C}^{k} k_{\mu}+\mathcal{C}^{m} m_{\mu}+\mathcal{C}^{\bar{m}} \bar{m}_{\mu}
$$

where we already used $\mathcal{C}^{n}=0$ because $k^{\mu} \mathcal{C}_{\mu}=0$ as in Eq. (3.9). Now we compare Eq. (B.16) with Eq. (B.15) and, using the fact that $\left(k_{\mu}, m_{\mu}, \bar{m}_{\mu}\right)$ constitute a basis we find the system of equations

$$
\begin{aligned}
2 \mathcal{C}^{k} & =2 \alpha_{k k}(\bar{v} \cdot k)+\alpha_{k m}(\bar{v} \cdot m)+\alpha_{k \bar{m}}(\bar{v} \cdot \bar{m}) \\
2 \mathcal{C}^{m} & =2 \alpha_{m m}(\bar{v} \cdot m)+\alpha_{k m}(\bar{v} \cdot k)+\alpha_{m \bar{m}}(\bar{v} \cdot \bar{m}) \\
2 \mathcal{C}^{\bar{m}} & =2 \alpha_{\bar{m} \bar{m}}(\bar{v} \cdot \bar{m})+\alpha_{k \bar{m}}(v \cdot k)+\alpha_{m \bar{m}}(\bar{v} \cdot m)
\end{aligned}
$$

which can be inverted for $\alpha_{k k}, \alpha_{m m}$ and $\alpha_{\bar{m} \bar{m}}$ in the case of $(v \cdot k) \neq 0$

$$
\begin{aligned}
& \alpha_{k m}= \frac{1}{(\bar{v} \cdot k)}\left[2 \mathcal{C}^{m}-2 \alpha_{m m}(\bar{v} \cdot m)-\alpha_{m \bar{m}}(\bar{v} \cdot \bar{m})\right] \\
& \alpha_{k \bar{m}}=\frac{1}{(\bar{v} \cdot k)}\left[2 \mathcal{C}^{\bar{m}}-2 \alpha_{\bar{m} \bar{m}}(\bar{v} \cdot \bar{m})-\alpha_{m \bar{m}}(\bar{v} \cdot m)\right] \\
& \alpha_{k k}=\frac{1}{(\bar{v} \cdot k)^{2}}\left[\mathcal{C}^{k}(\bar{v} \cdot k)-\mathcal{C}^{m}(\bar{v} \cdot m)-\mathcal{C}^{\bar{m}}(\bar{v} \cdot \bar{m})+\right. \\
&\left.\quad+\alpha_{m m}(\bar{v} \cdot m)^{2}+\alpha_{\bar{m} \bar{m}}(\bar{v} \cdot \bar{m})^{2}+\alpha_{m \bar{m}}(\bar{v} \cdot m)(\bar{v} \cdot \bar{m})\right]
\end{aligned}
$$

so that $\alpha_{k k}, \alpha_{k m}, \alpha_{k \bar{m}}$ are entirely written in terms of $\mathcal{C}^{k}, \mathcal{C}^{m}, \mathcal{C}^{\bar{m}}$ and the other free coefficients $\alpha_{m m}, \alpha_{\bar{m} \bar{m}}, \alpha_{m \bar{m}}$. The assumption $(v \cdot k) \neq 0$ is well justified because

$$
k^{\mu} \bar{v}_{\mu}=k^{\mu} \bar{\nabla}_{\mu} \bar{\varphi}=\frac{\mathrm{d} \bar{\varphi}}{\mathrm{d} \lambda},
$$

and we expect the smooth - slowly varying profile of the scalar field to change along the geodesics.

Using the gauge freedom associated to $\sigma_{\mu}$ one can fix the three independent components of $\mathcal{C}^{\mu}$ on the null tetrad basis, namely $\mathcal{C}^{k}, \mathcal{C}^{m}$ and $\mathcal{C}^{\bar{m}}$. The transformation law of $\mathcal{C}^{\mu}$ under $x^{\mu} \rightarrow x^{\mu}+\sigma^{\mu}$ is given in the main text. Doing so means that $\left(\alpha_{k k}, \alpha_{k m}, \alpha_{k \bar{m}}\right)$ can be entirely written in terms of $\left(\mathcal{C}^{k}, \mathcal{C}^{m}, \mathcal{C}^{\bar{m}}\right)$ and $\left(\alpha_{m m}, \alpha_{\bar{m} \bar{m}}, \alpha_{m \bar{m}}\right)$ through Eqs. (B.20) - (B.22). Note that we can choose $\left(\mathcal{C}^{k}, \mathcal{C}^{m}, \mathcal{C}^{\bar{m}}\right)$ such that $\alpha_{k k}=\alpha_{k m}=\alpha_{k \bar{m}}=0$ or not. In the first case one removes directly the vector and scalar-longitudinal modes form $\mathcal{A}_{\mu \nu}$ but also has to be careful to remove the same modes in the first-order stress energy tensor, $t_{\mu \nu}$. This can be achieved using the equations of motion Eqs. (B.40) - (B.45). In the second case, $\left(\alpha_{k k}, \alpha_{k m}, \alpha_{k \bar{m}} \neq 0\right)$, then it is still possible to decouple the dynamics of the physical modes, i.e. $\left(\alpha_{m m}, \alpha_{\bar{m} \bar{m}}, \alpha_{m \bar{m}}\right)$ from the three gauge ones $\left(\alpha_{k k}, \alpha_{k m}, \alpha_{k \bar{m}}\right)$. We show this second procedure in Appendix B.2.

\section{B.2 Decoupling of amplitude's evolution equations}

In the main text we computed the equations of motions for the amplitude and polarization tensor of a GW containing only the spin-2 and transverse spin-0 mode. In particular, we claimed that the amplitude of these modes satisfy Eq. (3.29) and Eq. (3.30) while, the total polarization tensor satisfies Eq. (3.33). Here we show how to separate the evolution equations 
of the physical modes from the spurious ones starting from the general decomposition of $\mathcal{A}_{\mu \nu}$ over the null tetrad basis, namely Eq. (B.11). In this section we use primes to denote quantities after the removal of the gauge modes $\left(\mathcal{A}_{\mu \nu}^{\prime}, \mathcal{A}^{\prime}, \mathbf{e}_{\mu \nu}^{\prime}, ..\right)$ to make a distinction with the previous part of this Appendix. In the main text from section 3.3 on, we always use $\mathcal{A}_{\mu \nu}^{\prime}$ and $\mathbf{e}_{\mu \nu}^{\prime}$ even though primes are omitted.

We start by plugging the decomposition of $\mathcal{A}_{\mu \nu}$ (B.11) in Eq. (3.13)

$$
\Theta_{\mu \nu}^{C D} \mathcal{B}_{C D}+2 \alpha_{C D} k^{\rho} \bar{\nabla}_{\rho} \Theta_{\mu \nu}^{C D}=t_{\mu \nu}
$$

where

$$
\mathcal{B}_{C D}=2 k^{\rho} \bar{\nabla}_{\rho} \alpha_{C D}+\alpha_{C D} \bar{\nabla}_{\rho} k^{\rho}
$$

In order obtain separate equations for the different modes, we contract Eq. (B.24) with $\Theta_{A B}^{\mu \nu}$ for different $A, B=\{n, k, n, \bar{m}\}$. Using the properties (B.6), we find

$$
\begin{aligned}
0 & =\Theta_{k k}^{\mu \nu} t_{\mu \nu} \\
0 & =\Theta_{k m}^{\mu \nu} t_{\mu \nu} \\
0 & =\Theta_{k m}^{\mu \nu} t_{\mu \nu} \\
0 & =\Theta_{k \bar{m}}^{\mu \nu} t_{\mu \nu} \\
\mathcal{B}_{\bar{m} \bar{m}}+4 \alpha_{\bar{m} \bar{m}} k^{\rho} m^{\mu} \bar{\nabla}_{\rho} \bar{m}_{\mu} & =\Theta_{m m}^{\mu \nu} t_{\mu \nu} \\
\mathcal{B}_{m m}+4 \alpha_{m m} k^{\rho} \bar{m}^{\mu} \bar{\nabla}_{\rho} m_{\mu} & =\Theta_{\bar{m} \bar{m}}^{\mu \nu} t_{\mu \nu} \\
\mathcal{B}_{m \bar{m}} & =2 \Theta_{m \bar{m}}^{\mu \nu} t_{\mu \nu} \\
-\frac{1}{2} \mathcal{B}_{k \bar{m}}-\alpha_{k \bar{m}} k^{\rho} m^{\mu} \bar{\nabla}_{\rho} \bar{m}_{\mu}+\alpha_{m \bar{m}} k^{\rho} n^{\mu} \bar{\nabla}_{\rho} m_{\mu}+2 \alpha_{\bar{m} \bar{m}} k^{\rho} n^{\mu} \bar{\nabla}_{\rho} \bar{m}_{\mu} & =\Theta_{n m}^{\mu \nu} t_{\mu \nu} \\
-\frac{1}{2} \mathcal{B}_{k m}-\alpha_{k m} k^{\rho} \bar{m}^{\mu} \bar{\nabla}_{\rho} m_{\mu}+\alpha_{m \bar{m}} k^{\rho} n^{\mu} \bar{\nabla}_{\rho} \bar{m}_{\mu}+2 \alpha_{m m} k^{\rho} n^{\mu} \bar{\nabla}_{\rho} m_{\mu} & =\Theta_{n \bar{m}}^{\mu \nu} t_{\mu \nu} \\
\mathcal{B}_{k k}-2 \alpha_{k m} k^{\rho} n^{\mu} \bar{\nabla}_{\rho} m_{\mu}-2 \alpha_{k \bar{m}} k^{\rho} n^{\mu} \bar{\nabla}_{\rho} \bar{m}_{\mu} & =\Theta_{n n}^{\mu \nu} t_{\mu \nu}
\end{aligned}
$$

We can decompose the stress energy tensor on the null tetrad basis as well

$$
t_{\mu \nu}=\mathcal{T}_{C D} \Theta_{\mu \nu}^{C D}
$$

with $C, D \in(k, m, \bar{m})$ because $t_{\mu \nu}$ is such that $k^{\mu} t_{\mu \nu}=0$ so that its component on the basis $z_{a}^{\mu}$ proportional to $n^{\mu}$ have to vanish. We can relate the coefficients $\mathcal{T}_{C D}$ to suitable contractions of $t_{\mu \nu}$ with the elements $\Theta_{\mu \nu}^{C D}$

$$
\begin{aligned}
& \Theta_{m \bar{m}}^{\mu \nu} t_{\mu \nu}=\frac{1}{2} \mathcal{T}_{m \bar{m}} \quad \Theta_{\bar{m} \bar{m}}^{\mu \nu} t_{\mu \nu}=\mathcal{T}_{m m} \quad \Theta_{m m}^{\mu \nu} t_{\mu \nu}=\mathcal{T}_{\bar{m} \bar{m}} \\
& \Theta_{m n}^{\mu \nu} t_{\mu \nu}=-\frac{1}{2} \mathcal{T}_{k \bar{m}} \quad \Theta_{\bar{m} n}^{\mu \nu} t_{\mu \nu}=-\frac{1}{2} \mathcal{T}_{k m} \quad \Theta_{n n}^{\mu \nu} t_{\mu \nu}=\mathcal{T}_{k k},
\end{aligned}
$$

while the other components are null. Since $t_{\mu \nu}$ is transverse the following relations hold

$$
\Theta_{k k}^{\mu \nu} t_{\mu \nu}=0 \quad \Theta_{k m}^{\mu \nu} t_{\mu \nu}=0 \quad \Theta_{k \bar{m}}^{\mu \nu} t_{\mu \nu}=0
$$


so that we see that Eqs. (B.26) - (B.29) are compatible with the condition $k^{\mu} t_{\mu \nu}=0$. Eqs. (B.30) - (B.35) in order become

$$
\begin{aligned}
& \mathcal{B}_{m m}+4 \alpha_{m m} k^{\rho} \bar{m}^{\mu} \bar{\nabla}_{\rho} m_{\mu}=\mathcal{T}_{m m} \\
& \mathcal{B}_{\bar{m} \bar{m}}-4 \alpha_{\bar{m} \bar{m}} k^{\rho} \bar{m}^{\mu} \bar{\nabla}_{\rho} m_{\mu}=\mathcal{T}_{\bar{m} \bar{m}} \\
& \mathcal{B}_{m \bar{m}}=\mathcal{T}_{m \bar{m}} \\
& \mathcal{B}_{k \bar{m}}+2 \alpha_{k \bar{m}} k^{\rho} m^{\mu} \bar{\nabla}_{\rho} \bar{m}_{\mu}-2 \alpha_{m \bar{m}} k^{\rho} n^{\mu} \bar{\nabla}_{\rho} m_{\mu}-4 \alpha_{\bar{m} \bar{m}} k^{\rho} n^{\mu} \bar{\nabla}_{\rho} \bar{m}_{\mu}=\mathcal{T}_{k \bar{m}} \\
& \mathcal{B}_{k m}+2 \alpha_{k m} k^{\rho} \bar{m}^{\mu} \bar{\nabla}_{\rho} m_{\mu}-2 \alpha_{m \bar{m}} k^{\rho} n^{\mu} \bar{\nabla}_{\rho} \bar{m}_{\mu}-4 \alpha_{m m} k^{\rho} n^{\mu} \bar{\nabla}_{\rho} m_{\mu}=\mathcal{T}_{k m} \\
& \mathcal{B}_{k k}-2 \alpha_{k m} k^{\rho} n^{\mu} \bar{\nabla}_{\rho} m_{\mu}-2 \alpha_{k \bar{m}} k^{\rho} n^{\mu} \bar{\nabla}_{\rho} \bar{m}_{\mu}=\mathcal{T}_{k k}
\end{aligned}
$$

We are interested in the evolution equation of $\mathcal{A}_{\mu \nu}$ after the removal of the modes $k k$, $k m$ and $k \bar{m}$, i.e. $\mathcal{A}_{\mu \nu}^{\prime}$. The decomposition of $\mathcal{A}_{\mu \nu}^{\prime}$ on the basis $z_{a}^{\mu}$ is given by

$$
\mathcal{A}_{\mu \nu}^{\prime}=\alpha_{m m} \Theta_{\mu \nu}^{m m}+\alpha_{\bar{m} \bar{m}} \Theta_{\mu \nu}^{\bar{m} \bar{m}}+\alpha_{m \bar{m}} \Theta_{\mu \nu}^{m \bar{m}}=\alpha_{E F} \Theta_{\mu \nu}^{E F}
$$

with $E, F=\{m, \bar{m}\}$. We can find the equation of motion of $\mathcal{A}_{\mu \nu}^{\prime}$ in two ways: either exploiting the gauge freedom associated to $\sigma_{\mu}$ as described in B.1 on $\mathcal{A}_{\mu \nu}$ or by moving the terms proportional to $\Theta_{\mu \nu}^{k k}, \Theta_{\mu \nu}^{k m}$ and $\Theta_{\mu \nu}^{k \bar{m}}$ in Eq. (B.24) from the LHS to its RHS and use the equations just derived. Both of the procedures lead to the same result and here we show the second methodology. We also choose a parallel transported null-tetrad along the rays to make the computations less involved, so that Eqs. (B.40) - (B.45) reduce to

$$
\mathcal{B}_{m m}=\mathcal{T}_{m m}, \quad \mathcal{B}_{\bar{m} \bar{m}}=\mathcal{T}_{\bar{m} \bar{m}}, \quad \mathcal{B}_{m \bar{m}}=\mathcal{T}_{m \bar{m}}, \quad \mathcal{B}_{k \bar{m}}=\mathcal{T}_{k \bar{m}}, \quad \mathcal{B}_{k m}=\mathcal{T}_{k m}, \quad \mathcal{B}_{k k}=\mathcal{T}_{k k}
$$

In the case of parallel transported null-tetrad Eq (B.24) is given by

$$
\Theta_{\mu \nu}^{C D} \mathcal{B}_{C D}=t_{\mu \nu}=\mathcal{T}_{C D} \Theta_{\mu \nu}^{C D},
$$

where $C, D=\{k, m, \bar{m}\}$. Now we move from the LHS to the RHS the terms proportional to $\Theta_{\mu \nu}^{k k}, \Theta_{\mu \nu}^{k m}$ and $\Theta_{\mu \nu}^{k \bar{m}}$ in Eq (B.48)

$$
\begin{aligned}
\Theta_{\mu \nu}^{E F} \mathcal{B}_{E F}=\Theta_{\mu \nu}^{m m} \mathcal{B}_{m m}+ & \Theta_{\mu \nu}^{\bar{m} \bar{m}} \mathcal{B}_{\bar{m} \bar{m}}+\Theta_{\mu \nu}^{m \bar{m}} \mathcal{B}_{m \bar{m}}= \\
= & \mathcal{T}_{C D} \Theta_{\mu \nu}^{C D}-\Theta_{\mu \nu}^{k k} \mathcal{B}_{k k}+\Theta_{\mu \nu}^{k m} \mathcal{B}_{k \bar{m}}+\Theta_{\mu \nu}^{k \bar{m}} \mathcal{B}_{k \bar{m}}= \\
= & \Theta_{\mu \nu}^{m m} \mathcal{T}_{m m}+\Theta_{\mu \nu}^{\bar{m} \bar{m}} \mathcal{T}_{\bar{m} \bar{m}}+\Theta_{\mu \nu}^{m \bar{m}} \mathcal{T}_{m \bar{m}}+\Theta_{\mu \nu}^{k m}\left(\mathcal{T}_{k m}-\mathcal{B}_{k m}\right)+ \\
& +\Theta_{\mu \nu}^{k \bar{m}}\left(\mathcal{T}_{k \bar{m}}-\mathcal{B}_{k \bar{m}}\right)+\Theta_{\mu \nu}^{k k}\left(\mathcal{T}_{k k}-\mathcal{B}_{k k}\right)= \\
= & \Theta_{\mu \nu}^{m m} \mathcal{T}_{m m}+\Theta_{\mu \nu}^{\bar{m} \bar{m}} \mathcal{T}_{\bar{m} \bar{m}}+\Theta_{\mu \nu}^{m \bar{m}} \mathcal{T}_{m \bar{m}}
\end{aligned}
$$

where in the last step we used Eqs. (B.47) and $E, F=\{m, \bar{m}\}$. We define the first-order stress energy tensor deprived of the gauge modes

$$
t_{\mu \nu}^{\prime}:=\Theta_{\mu \nu}^{m m} \mathcal{T}_{m m}+\Theta_{\mu \nu}^{\bar{m} \bar{m}} \mathcal{T}_{\bar{m} \bar{m}}+\Theta_{\mu \nu}^{m \bar{m}} \mathcal{T}_{m \bar{m}} .
$$

It is possible to show that this is the same form of $t_{\mu \nu}^{\prime}$ one would find by imposing the gauge fixing described in B.1 to eliminate $\alpha_{k k}, \alpha_{k m}$ and $\alpha_{k \bar{m}}$. Finally, we find the equation of motion of $\mathcal{A}_{\mu \nu}^{\prime}$ by realizing that

$$
\begin{aligned}
\Theta_{\mu \nu}^{E F} \mathcal{B}_{E F} & =\Theta_{\mu \nu}^{E F}\left(2 k^{\rho} \bar{\nabla}_{\rho} \alpha_{E F}+\alpha_{E F} \bar{\nabla}_{\rho} k^{\rho}\right)=2 k^{\rho} \bar{\nabla}_{\rho}\left(\alpha_{E F} \Theta_{\mu \nu}^{E F}\right)+\alpha_{E F} \Theta_{\mu \nu}^{E F} \bar{\nabla}_{\rho} k^{\rho} \\
& =2 k^{\rho} \bar{\nabla}_{\rho} \mathcal{A}_{\mu \nu}^{\prime}+\mathcal{A}_{\mu \nu}^{\prime} \bar{\nabla}_{\rho} k^{\rho},
\end{aligned}
$$


where we used the definition of $\mathcal{B}_{C D}$ given in Eq. (B.25) and the fact that the tetrad is parallel transported so that $k^{\rho} \bar{\nabla}_{\rho} \Theta_{\mu \nu}^{E F}=0$. Combining Eq. (B.51) and Eq. (B.49) yields

$$
2 k^{\rho} \bar{\nabla}_{\rho} \mathcal{A}_{\mu \nu}^{\prime}+\mathcal{A}_{\mu \nu}^{\prime} \bar{\nabla}_{\rho} k^{\rho}=t_{\mu \nu}^{\prime}
$$

which is the desired result. The equation of motion of $\mathbf{e}_{\mu \nu}^{\prime}$ can then be found by contracting Eq. (B.52) with $\mathbf{e}^{\prime \mu \nu}$

$$
\begin{aligned}
\mathbf{e}^{\prime \mu \nu}\left[2 k^{\rho} \bar{\nabla}_{\rho} \mathcal{A}_{\mu \nu}^{\prime}+\mathcal{A}_{\mu \nu}^{\prime} \bar{\nabla}_{\rho} k^{\rho}\right] & =t_{\mu \nu}^{\prime} \mathbf{e}^{\prime \mu \nu} \\
2 k^{\rho} \bar{\nabla}_{\rho} \mathcal{A}^{\prime}+\mathcal{A}^{\prime} \bar{\nabla}_{\rho} k^{\rho} & =t_{\mu \nu}^{\prime} \mathbf{e}^{\prime \mu \nu}
\end{aligned}
$$

where we used $\mathbf{e}^{\prime \mu \nu} \mathbf{e}_{\mu \nu}^{\prime}=1$ so that $\mathbf{e}^{\prime \mu \nu} \mathcal{A}_{\mu \nu}^{\prime}=\mathcal{A}^{\prime}$. Subtracting Eq. (B.52) and Eq. (B.53) and using $\mathcal{A}_{\mu \nu}^{\prime}=\mathcal{A}^{\prime} \mathbf{e}_{\mu \nu}^{\prime}$ yields

$$
k^{\rho} \bar{\nabla}_{\rho} \mathbf{e}_{\mu \nu}^{\prime}=\frac{1}{2 \mathcal{A}^{\prime}}\left[t_{\mu \nu}^{\prime}-\mathbf{e}_{\mu \nu}^{\prime}\left(t_{\rho \sigma}^{\prime} \mathbf{e}^{\prime \rho \sigma}\right)\right],
$$

which is Eq. (3.33). In the case of a stress-energy tensor given by

$$
t_{\mu \nu} \equiv\left\{\tau^{(A)} \mathcal{A}_{\mu \nu}+\tau^{(B)}\left(k_{\mu} \mathcal{C}_{\nu}+k_{\nu} \mathcal{C}_{\mu}\right)+\tau^{(C)}\left[k_{\mu} \bar{v}_{\nu}+k_{\nu} \bar{v}_{\mu}-\bar{g}_{\mu \nu}\left(k_{\rho} \bar{v}^{\rho}\right)\right]\right\},
$$

we can use Eq. (B.37) to find the components of $t_{\mu \nu}^{\prime}$ in the null tetrad basis explicitly. These are given by

$$
\begin{aligned}
& \mathcal{T}_{m \bar{m}}=\tau^{(A)} \alpha_{m \bar{m}}-2 \tau^{(C)}\left(k_{\rho} \bar{v}^{\rho}\right), \\
& \mathcal{T}_{m m}=\tau^{(A)} \alpha_{m m}, \\
& \mathcal{T}_{\bar{m} \bar{m}}=\tau^{(A)} \alpha_{\bar{m} \bar{m}},
\end{aligned}
$$

so that $t_{\mu \nu}^{\prime}$, defined as in Eq. (B.50), takes the form

$$
\begin{aligned}
t_{\mu \nu}^{\prime} & =\tau^{(A)} \alpha_{m m} \Theta_{\mu \nu}^{m m}+\tau^{(A)} \alpha_{\bar{m} \bar{m}} \Theta_{\mu \nu}^{\bar{m} \bar{m}}+\left(\tau^{(A)} \alpha_{m \bar{m}}-2 \tau^{(C)}\left(k_{\rho} \bar{v}^{\rho}\right)\right) \Theta_{\mu \nu}^{m \bar{m}} \\
& =\tau^{(A)} \mathcal{A}_{\mu \nu}^{\prime}-2 \tau^{(C)}\left(k_{\rho} \bar{v}^{\rho}\right) \Theta_{\mu \nu}^{m \bar{m}}=\tau^{(A)} \mathcal{A}_{\mu \nu}^{\prime}-\sqrt{2} \tau^{(C)}\left(k_{\rho} \bar{v}^{\rho}\right) \mathbf{e}_{\mu \nu}^{S},
\end{aligned}
$$

where, in the last step, we used $\mathbf{e}_{\mu \nu}^{S}=\sqrt{2} \Theta_{\mu \nu}^{m \bar{m}}$ as claimend in Eq. (B.12). Using these expressions Eqs. (B.40), (B.41) and (B.42) become

$$
\begin{aligned}
2 k^{\rho} \bar{\nabla}_{\rho} \alpha_{m m}+\alpha_{m m} \bar{\nabla}_{\rho} k^{\rho} & =\tau^{(A)} \alpha_{m m}, \\
2 k^{\rho} \bar{\nabla}_{\rho} \alpha_{\bar{m} \bar{m}}+\alpha_{\bar{m} \bar{m}} \bar{\nabla}_{\rho} k^{\rho} & =\tau^{(A)} \alpha_{\bar{m} \bar{m}}, \\
2 k^{\rho} \bar{\nabla}_{\rho} \alpha_{m \bar{m}}+\alpha_{m \bar{m}} \bar{\nabla}_{\rho} k^{\rho} & =\tau^{(A)} \alpha_{m \bar{m}}-2 \tau^{(C)}\left(k_{\rho} \bar{v}^{\rho}\right) .
\end{aligned}
$$

Using Eqs. (B.13) and (B.12) into the sum and the difference of Eq. (B.60) and Eq. (B.61), and setting $\mathcal{A}^{+}=\mathcal{A}^{\times}=\mathcal{A}^{T}$, then one may recover the evolution equation of $\mathcal{A}^{T}$ present in the main text, i.e. Eq. (3.30). Eq. (B.62) is in turn equivalent to Eq. (3.29) after substituting $\alpha_{m \bar{m}}=\sqrt{2} \mathcal{A}^{S}$.

Now we turn to the evolution equation of the polarization tensor after the removal of the modes $k k, k m$ and $k \bar{m}$, Eq. (B.54). Note that Eqs. (B.13) and (B.12) allow us to decompose $\mathcal{A}_{\mu \nu}^{\prime}$ both on the basis $\Theta_{\mu \nu}^{E F}$ or $\left(\mathbf{e}_{\mu \nu}^{+}, \mathbf{e}_{\mu \nu}^{\times}, \mathbf{e}_{\mu \nu}^{S}\right)$ equivalently, i.e.

$$
\mathcal{A}_{\mu \nu}^{\prime}=\alpha_{m m} \Theta_{\mu \nu}^{m m}+\alpha_{\bar{m} \bar{m}} \Theta_{\mu \nu}^{\bar{m} \bar{m}}+\alpha_{m \bar{m}} \Theta_{\mu \nu}^{m \bar{m}}=\mathcal{A}^{+} \mathbf{e}_{\mu \nu}^{+}+\mathcal{A}^{\times} \mathbf{e}_{\mu \nu}^{\times}+\mathcal{A}^{S} \mathbf{e}_{\mu \nu}^{S} .
$$


For $t_{\mu \nu}^{\prime}$ of the form in Eq. (B.59) we have

$$
\begin{aligned}
\mathbf{e}^{\prime \mu \nu} t_{\mu \nu}^{\prime} & =\tau^{(A)} \mathcal{A}^{\prime}-\sqrt{2} \tau^{(C)}\left(k_{\rho} \bar{v}^{\rho}\right) \mathbf{e}^{\prime \mu \nu} \mathbf{e}_{\mu \nu}^{S}=\tau^{(A)} \mathcal{A}^{\prime}-\sqrt{2} \tau^{(C)}\left(k_{\rho} \bar{v}^{\rho}\right) \frac{\mathcal{A}^{\prime \mu \nu} \mathbf{e}_{\mu \nu}^{S}}{\mathcal{A}^{\prime}} \\
& =\tau^{(A)} \mathcal{A}^{\prime}-\sqrt{2} \tau^{(C)}\left(k_{\rho} \bar{v}^{\rho}\right) \frac{\mathcal{A}^{\prime \mu \nu} \mathbf{e}_{\mu \nu}^{S}}{\mathcal{A}^{\prime}}=\tau^{(A)} \mathcal{A}^{\prime}-\sqrt{2} \tau^{(C)}\left(k_{\rho} \bar{v}^{\rho}\right) \frac{\mathcal{A}^{S}}{\mathcal{A}^{\prime}}
\end{aligned}
$$

so that

$$
\begin{aligned}
t_{\mu \nu}^{\prime}-\mathbf{e}_{\mu \nu}^{\prime}\left(t_{\rho \sigma}^{\prime} \mathbf{e}^{\prime \rho \sigma}\right) & =\sqrt{2} \tau^{(C)}\left(k_{\rho} \bar{v}^{\rho}\right) \frac{\mathcal{A}^{S}}{\mathcal{A}^{\prime}} \mathbf{e}_{\mu \nu}^{\prime}-\sqrt{2} \tau^{(C)}\left(k_{\rho} \bar{v}^{\rho}\right) \mathbf{e}_{\mu \nu}^{S}= \\
& =\sqrt{2} \tau^{(C)}\left(k_{\rho} \bar{v}^{\rho}\right)\left[\frac{\mathcal{A}^{S}}{\mathcal{A}^{\prime 2}} \mathcal{A}_{\mu \nu}^{\prime}-\mathbf{e}_{\mu \nu}^{S}\right]
\end{aligned}
$$

and

$$
k^{\rho} \bar{\nabla}_{\rho} \mathbf{e}_{\mu \nu}^{\prime}=\frac{1}{2 \mathcal{A}^{\prime}}\left[t_{\mu \nu}^{\prime}-\mathbf{e}_{\mu \nu}^{\prime}\left(t_{\rho \sigma}^{\prime} \mathbf{e}^{\prime \rho \sigma}\right)\right]=\frac{\left(k_{\rho} \bar{v}^{\rho}\right) \tau^{(C)}}{\sqrt{2} \mathcal{A}^{\prime}}\left[\frac{\mathcal{A}^{S}}{\mathcal{A}^{\prime 2}} \mathcal{A}_{\mu \nu}^{\prime}-\mathbf{e}_{\mu \nu}^{S}\right]
$$

which is the final step in Eq. (3.33). Note that, in general, the LHS of Eq. (B.66) is non zero even if the null tetrad is chosen parallel transported along the rays because

$$
k^{\rho} \bar{\nabla}_{\rho} \mathbf{e}_{\mu \nu}^{\prime}=k^{\rho} \bar{\nabla}_{\rho}\left(\frac{\mathcal{A}_{\mu \nu}^{\prime}}{\mathcal{A}^{\prime}}\right)=k^{\rho} \bar{\nabla}_{\rho}\left(\frac{\alpha_{E F}^{\prime} \Theta_{\mu \nu}^{E F}}{\mathcal{A}^{\prime}}\right)=k^{\rho} \Theta_{\mu \nu}^{E F} \bar{\nabla}_{\rho}\left(\frac{\alpha_{E F}}{\mathcal{A}^{\prime}}\right)
$$

where $E, F=\{m, \bar{m}\}$. The Eq. above states that the transport properties of $\mathbf{e}_{\mu \nu}^{\prime}$ depend on the evolution equations of the amplitude of the modes present. In general $\mathbf{e}_{\mu \nu}^{\prime}$ is not parallel transported whenever the amplitudes of the single components of the wave decay differently between each other. In the theory we considered, the spin- 0 mode satisfies an evolution equation ( i.e. Eq. (3.29)) which is different from the one of the two tensor modes (i.e. Eq. (3.30)). This fact has as consequence that the total polarization is not parallel transported along the GW's geodesic. In the case of GR, where the RHS of Eq. (B.66) is zero, we see that also the LHS is zero. In fact, in GR the mode $m \bar{m}$ is not present and the plus and cross polarization modes have the same amplitude so that $\mathcal{A}_{\mu \nu}^{\prime}=\mathcal{A}^{+} \mathbf{e}_{\mu \nu}^{+}+\mathcal{A}^{\times} \mathbf{e}_{\mu \nu}^{\times}=\mathcal{A}^{T}\left(\mathbf{e}_{\mu \nu}^{+}+\mathbf{e}_{\mu \nu}^{\times}\right)$ and $\mathcal{A}^{\prime}=\sqrt{2} \mathcal{A}^{T}$. Thus

$$
k^{\rho} \Theta_{\mu \nu}^{E F} \bar{\nabla}_{\rho}\left(\frac{\alpha_{E F}}{\mathcal{A}^{\prime}}\right)=k^{\rho}\left(\mathbf{e}_{\mu \nu}^{+}+\mathbf{e}_{\mu \nu}^{\times}\right) \bar{\nabla}_{\rho}\left(\frac{\mathcal{A}^{T}}{\sqrt{2} \mathcal{A}^{T}}\right)=k^{\rho}\left(\mathbf{e}_{\mu \nu}^{+}+\mathbf{e}_{\mu \nu}^{\times}\right) \bar{\nabla}_{\rho}\left(\frac{1}{\sqrt{2}}\right)=0 .
$$

\section{References}

[1] N. A. Bahcall, J. P. Ostriker, S. Perlmutter, and P. J. Steinhardt, "The Cosmic triangle: Assessing the state of the universe", Science 284 (1999) 1481-1488, arXiv:astro-ph/9906463 [astro-ph].

[2] S. Weinberg, "The Cosmological Constant Problem", Rev. Mod. Phys. 61 (1989) 1-23. [,569(1988)].

[3] W. L. Freedman, "Cosmology at a Crossroads", Nat. Astron. 1 (2017) 0121, arXiv:1706.02739 [astro-ph.CO]. 
[4] J. Frieman, M. Turner, and D. Huterer, "Dark Energy and the Accelerating Universe", Ann. Rev. Astron. Astrophys. 46 (2008) 385-432, arXiv:0803.0982 [astro-ph].

[5] T. Clifton, P. G. Ferreira, A. Padilla, and C. Skordis, "Modified Gravity and Cosmology", Phys. Rept. 513 (2012) 1-189, arXiv:1106.2476 [astro-ph.CO].

[6] A. Joyce, B. Jain, J. Khoury, and M. Trodden, "Beyond the Cosmological Standard Model", Phys. Rept. 568 (2015) 1-98, arXiv:1407.0059 [astro-ph.CO].

[7] C. Burrage and J. Sakstein, "Tests of Chameleon Gravity", Living Rev. Rel. 21 no. 1, (2018) 1, arXiv:1709.09071 [astro-ph.C0].

[8] E. Babichev and C. Deffayet, "An introduction to the Vainshtein mechanism", Class. Quant. Grav. 30 (2013) 184001, arXiv:1304.7240 [gr-qc].

[9] N. Yunes, K. Yagi, and F. Pretorius, "Theoretical Physics Implications of the Binary Black-Hole Mergers GW150914 and GW151226", Phys. Rev. D94 no. 8, (2016) 084002, arXiv:1603.08955 [gr-qc].

[10] K. Pardo, M. Fishbach, D. E. Holz, and D. N. Spergel, "Limits on the number of spacetime dimensions from GW170817", JCAP 1807 no. 07, (2018) 048, arXiv: 1801.08160 [gr-qc].

[11] M. Lagos, M. Fishbach, P. Landry, and D. E. Holz, "Standard sirens with a running Planck mass", Phys. Rev. D99 no. 8, (2019) 083504, arXiv:1901.03321 [astro-ph.C0].

[12] The LIGO Scientific, Virgo, B. P. Abbott et al., "Tests of General Relativity with GW170817", arXiv:1811.00364 [gr-qc].

[13] A. Nishizawa and S. Arai, "Generalized framework for testing gravity with gravitational-wave propagation. III. Future prospect", arXiv:1901.08249 [gr-qc].

[14] E. Di Valentino, D. E. Holz, A. Melchiorri, and F. Renzi, "The cosmological impact of future constraints on $H_{0}$ from gravitational-wave standard sirens", Phys. Rev. D98 no. 8, (2018) 083523, arXiv:1806.07463 [astro-ph.C0].

[15] B. Abbott et al., "GW170817: Observation of Gravitational Waves from a Binary Neutron Star Inspiral", Phys. Rev. Lett. 119 (2017) 161101, arXiv:1710.05832 [gr-qc].

[16] A. Goldstein et al., "An Ordinary Short Gamma-Ray Burst with Extraordinary Implications: Fermi-GBM Detection of GRB 170817A", Astrophys. J. 848 (2017) L14, arXiv:1710.05446 [astro-ph.HE].

[17] V. Savchenko et al., "INTEGRAL Detection of the First Prompt Gamma-Ray Signal Coincident with the Gravitational-wave Event GW170817", Astrophys. J. 848 (2017) L15, arXiv:1710.05449 [astro-ph.HE].

[18] B. P. Abbott et al., "Gravitational Waves and Gamma-rays from a Binary Neutron Star Merger: GW170817 and GRB 170817A", Astrophys. J. 848 (2017) L13, arXiv:1710.05834 [astro-ph.HE].

[19] B. P. Abbott et al., "Multi-messenger Observations of a Binary Neutron Star Merger", Astrophys. J. $\overline{848}$ no. 2, (2017) L12, arXiv:1710.05833 [astro-ph.HE].

[20] P. Creminelli and F. Vernizzi, "Dark Energy after GW170817 and GRB170817A", Phys. Rev. Lett. 119 (2017) 251302, arXiv:1710.05877 [astro-ph.C0].

[21] J. Sakstein and B. Jain, "Implications of the Neutron Star Merger GW170817 for Cosmological Scalar-Tensor Theories", Phys. Rev. Lett. 119 (2017) 251303, arXiv:1710.05893 [astro-ph.CO].

[22] J. M. Ezquiaga and M. Zumalacarregui, "Dark Energy After GW170817: Dead Ends and the Road Ahead", Phys. Rev. Lett. 119 (2017) 251304, arXiv:1710.05901 [astro-ph.C0]. 
[23] T. Baker, E. Bellini, P. G. Ferreira, M. Lagos, J. Noller, and I. Sawicki, "Strong constraints on cosmological gravity from GW170817 and GRB 170817A", Phys. Rev. Lett. 119 (2017) 251301, arXiv:1710.06394 [astro-ph.C0].

[24] L. Lombriser and A. Taylor, "Breaking a Dark Degeneracy with Gravitational Waves", JCAP 1603 (2016) 031, arXiv:1509.08458 [astro-ph.CO].

[25] D. Bettoni, J. M. Ezquiaga, K. Hinterbichler, and M. Zumalacarregui, "Speed of Gravitational Waves and the Fate of Scalar-Tensor Gravity", Phys. Rev. D95 no. 8, (2017) 084029, arXiv:1608.01982 [gr-qc].

[26] B. F. Schutz, "Determining the Hubble Constant from Gravitational Wave Observations", Nature 323 (1986) 310-311.

[27] D. E. Holz and S. A. Hughes, "Using gravitational-wave standard sirens", Astrophys. J. 629 (2005) 15-22, arXiv:astro-ph/0504616 [astro-ph].

[28] N. Dalal, D. E. Holz, S. A. Hughes, and B. Jain, "Short GRB and binary black hole standard sirens as a probe of dark energy", Phys. Rev. D74 (2006) 063006, arXiv:astro-ph/0601275 [astro-ph].

[29] C. L. MacLeod and C. J. Hogan, "Precision of Hubble constant derived using black hole binary absolute distances and statistical redshift information", Phys. Rev. D77 (2008) 043512, arXiv:0712.0618 [astro-ph].

[30] S. Nissanke, D. E. Holz, S. Hughes, N. Dalal, and J. L. Sievers, "Exploring short gamma-ray bursts as gravitational-wave standard sirens", Astrophys. J. 725 (2010) 496-514, arXiv:0904.1017 [astro-ph.CO].

[31] C. Cutler and D. E. Holz, "Ultra-high precision cosmology from gravitational waves", Phys. Rev. D80 (2009) 104009, arXiv:0906.3752 [astro-ph.CO].

[32] S. Camera and A. Nishizawa, "Beyond Concordance Cosmology with Magnification of Gravitational-Wave Standard Sirens", Phys. Rev. Lett. 110 no. 15, (2013) 151103, arXiv: 1303.5446 [astro-ph.CO].

[33] R. DAgostino and R. C. Nunes, "Probing observational bounds on scalar-tensor theories from standard sirens", Physical Review D 100 no. 4, (Aug, 2019) . http://dx.doi.org/10.1103/PhysRevD.100.044041.

[34] W. Del Pozzo, "Inference of the cosmological parameters from gravitational waves: application to second generation interferometers", Phys. Rev. D86 (2012) 043011, arXiv:1108.1317 [astro-ph.CO].

[35] B. S. Sathyaprakash, B. F. Schutz, and C. Van Den Broeck, "Cosmography with the Einstein Telescope", Class. Quant. Grav. 27 (2010) 215006, arXiv:0906.4151 [astro-ph.C0].

[36] W. Zhao, C. Van Den Broeck, D. Baskaran, and T. G. F. Li, "Determination of Dark Energy by the Einstein Telescope: Comparing with CMB, BAO and SNIa Observations", Phys. Rev. D83 (2011) 023005, arXiv: 1009.0206 [astro-ph.C0].

[37] S. R. Taylor and J. R. Gair, "Cosmology with the lights off: standard sirens in the Einstein Telescope era", Phys. Rev. D86 (2012) 023502, arXiv:1204.6739 [astro-ph.C0].

[38] A. Nishizawa, K. Yagi, A. Taruya, and T. Tanaka, "Cosmology with space-based gravitational-wave detectors - dark energy and primordial gravitational waves -", Phys. Rev. D85 (2012) 044047, arXiv:1110.2865 [astro-ph.C0].

[39] N. Tamanini, C. Caprini, E. Barausse, A. Sesana, A. Klein, and A. Petiteau, "Science with the space-based interferometer eLISA. III: Probing the expansion of the Universe using gravitational wave standard sirens", JCAP 1604 (2016) 002, arXiv:1601.07112 [astro-ph.Co]. 
[40] C. Caprini and N. Tamanini, "Constraining early and interacting dark energy with gravitational wave standard sirens: the potential of the eLISA mission", JCAP 1610 (2016) 006, arXiv: 1607.08755 [astro-ph.C0].

[41] The LISA Cosmology Working Group, E. Belgacem et al., "Testing modified gravity at cosmological distances with LISA standard sirens", JCAP 1907 no. 07, (2019) 024, arXiv:1906.01593 [astro-ph.CO].

[42] C. Deffayet and K. Menou, "Probing Gravity with Spacetime Sirens", Astrophys. J. 668 (2007) L143-L146, arXiv:0709.0003 [astro-ph].

[43] I. D. Saltas, I. Sawicki, L. Amendola, and M. Kunz, "Anisotropic Stress as a Signature of Nonstandard Propagation of Gravitational Waves", Phys. Rev. Lett. 113 (2014) 191101, arXiv:1406.7139 [astro-ph.C0].

[44] A. Nishizawa, "Generalized framework for testing gravity with gravitational-wave propagation. I. Formulation", Phys. Rev. D97 (2018) 104037, arXiv:1710.04825 [gr-qc].

[45] E. Belgacem, Y. Dirian, S. Foffa, and M. Maggiore, "Gravitational-wave luminosity distance in modified gravity theories", Phys. Rev. D97 (2018) 104066, arXiv:1712.08108 [astro-ph.CO].

[46] E. Belgacem, Y. Dirian, S. Foffa, and M. Maggiore, "Modified gravitational-wave propagation and standard sirens", Phys. Rev. D98 (2018) 023510, arXiv:1805.08731 [gr-qc].

[47] J. M. Ezquiaga and M. Zumalacrregui, "Dark Energy in light of Multi-Messenger Gravitational-Wave astronomy", Front. Astron. Space Sci. 5 (2018) 44, arXiv:1807.09241 [astro-ph.CO].

[48] R. A. Isaacson, "Gravitational Radiation in the Limit of High Frequency. I. The Linear Approximation and Geometrical Optics", Phys. Rev. 166 (1968) 1263-1271.

[49] R. A. Isaacson, "Gravitational Radiation in the Limit of High Frequency. II. Nonlinear Terms and the Ef fective Stress Tensor", Phys. Rev. 166 (1968) 1272-1279.

[50] C. W. Misner, K. S. Thorne, and J. A. Wheeler, Gravitation. W. H. Freeman, San Francisco, 1973.

[51] A. M. Anile, "Geometrical Optics in General Relativity: A Study of the Higher Order Corrections", J. Math. Phys. 17 (1976) 576-584.

[52] A. I. Harte, "Gravitational lensing beyond geometric optics: I. Formalism and observables", Gen. Rel. Grav. 51 no. 1, (2019) 14, arXiv:1808.06203 [gr-qc].

[53] S. R. Dolan, "Higher-order geometrical optics for electromagnetic waves on a curved spacetime", arXiv: 1801.02273 [gr-qc].

[54] R. Takahashi, T. Suyama, and S. Michikoshi, "Scattering of gravitational waves by the weak gravitational fields of lens objects", Astron. Astrophys. 438 (2005) L5, arXiv:astro-ph/0503343 [astro-ph].

[55] M. A. Oancea, C. F. Paganini, J. Joudioux, and L. Andersson, "An overview of the gravitational spin Hall effect", arXiv: 1904.09963 [gr-qc].

[56] S. Hou, X.-L. Fan, and Z.-H. Zhu, "Gravitational Lensing of Gravitational Waves: Rotation of Polarization Plane", Phys. Rev. D100 no. 6, (2019) 064028, arXiv:1907.07486 [gr-qc].

[57] C. Brans and R. H. Dicke, "Mach's Principle and a Relativistic Theory of Gravitation", Physical Review 124 (Nov., 1961) 925-935.

[58] C. Armendariz-Picon, V. F. Mukhanov, and P. J. Steinhardt, "Essentials of k essence", Phys. Rev. D63 (2001) 103510, arXiv:astro-ph/0006373 [astro-ph].

[59] A. Nicolis, R. Rattazzi, and E. Trincherini, "The Galileon as a local modification of gravity", Phys. Rev. D79 (2009) 064036, arXiv:0811.2197 [hep-th]. 
[60] G. W. Horndeski, "Second-order scalar-tensor field equations in a four-dimensional space", Int. J. Theor. Phys. 10 (1974) 363-384.

[61] C. Deffayet, X. Gao, D. A. Steer, and G. Zahariade, "From k-essence to generalised Galileons", Phys. Rev. D84 (2011) 064039, arXiv:1103.3260 [hep-th].

[62] T. Kobayashi, M. Yamaguchi, and J. Yokoyama, "Generalized G-inflation: Inflation with the most general second-order field equations", Prog. Theor. Phys. 126 (2011) 511-529, arXiv:1105.5723 [hep-th].

[63] M. Zumalacarregui and J. Garcia-Bellido, "Transforming gravity: from derivative couplings to matter to second-order scalar-tensor theories beyond the Horndeski Lagrangian", Phys. Rev. D89 (2014) 064046, arXiv:1308.4685 [gr-qc].

[64] J. Gleyzes, D. Langlois, F. Piazza, and F. Vernizzi, "Healthy theories beyond Horndeski", Phys. Rev. Lett. 114 (2015) 211101, arXiv:1404.6495 [hep-th].

[65] D. Langlois and K. Noui, "Degenerate higher derivative theories beyond Horndeski: evading the Ostrogradski instability", JCAP 1602 no. 02, (2016) 034, arXiv:1510.06930 [gr-qc].

[66] D. Langlois, "Dark Energy and Modified Gravity in Degenerate Higher-Order Scalar-Tensor (DHOST) theories: a review", arXiv:1811.06271 [gr-qc].

[67] M. Crisostomi, K. Koyama, and G. Tasinato, "Extended Scalar-Tensor Theories of Gravity", JCAP 1604 no. 04, (2016) 044, arXiv: 1602.03119 [hep-th].

[68] J. Ben Achour, D. Langlois, and K. Noui, "Degenerate higher order scalar-tensor theories beyond Horndeski and disformal transformations", Phys. Rev. D93 no. 12, (2016) 124005, arXiv:1602.08398 [gr-qc].

[69] J. Ben Achour, M. Crisostomi, K. Koyama, D. Langlois, K. Noui, and G. Tasinato, "Degenerate higher order scalar-tensor theories beyond Horndeski up to cubic order", JHEP 12 (2016) 100, arXiv:1608.08135 [hep-th].

[70] C. de Rham and A. Matas, "Ostrogradsky in Theories with Multiple Fields", JCAP 1606 no. 06, (2016) 041, arXiv: 1604.08638 [hep-th].

[71] D. Langlois, M. Mancarella, K. Noui, and F. Vernizzi, "Effective Description of Higher-Order Scalar-Tensor Theories", JCAP 1705 no. 05, (2017) 033, arXiv:1703.03797 [hep-th].

[72] C. Deffayet, O. Pujolas, I. Sawicki, and A. Vikman, "Imperfect Dark Energy from Kinetic Gravity Braiding", JCAP 1010 (2010) 026, arXiv: 1008.0048 [hep-th].

[73] M. Sasaki, "The Magnitude - Redshift relation in a perturbed Friedmann universe", Mon. Not. Roy. Astron. Soc. 228 (1987) 653-669.

[74] T. Pyne and M. Birkinshaw, "The luminosity distance in perturbed flrw spacetimes", Mon. Not. Roy. Astron. Soc. 348 (2004) 581, arXiv: astro-ph/0310841 [astro-ph].

[75] D. E. Holz and E. V. Linder, "Safety in numbers: Gravitational lensing degradation of the luminosity distance-redshift relation", Astrophys. J. 631 (2005) 678-688, arXiv:astro-ph/0412173 [astro-ph].

[76] C. Bonvin, R. Durrer, and M. A. Gasparini, "Fluctuations of the luminosity distance", Phys. Rev. D73 (2006) 023523, arXiv:astro-ph/0511183 [astro-ph]. [Erratum: Phys. Rev.D85,029901(2012)].

[77] L. Hui and P. B. Greene, "Correlated Fluctuations in Luminosity Distance and the (Surprising) Importance of Peculiar Motion in Supernova Surveys", Phys. Rev. D73 (2006) 123526, arXiv:astro-ph/0512159 [astro-ph].

[78] P. Laguna, S. L. Larson, D. Spergel, and N. Yunes, "Integrated Sachs-Wolfe Effect for Gravitational Radiation", Astrophys. J. 715 (2010) L12, arXiv:0905.1908 [gr-qc]. 
[79] V. Alba and J. Maldacena, "Primordial gravity wave background anisotropies", JHEP 03 (2016) 115, arXiv:1512.01531 [hep-th].

[80] C. R. Contaldi, "Anisotropies of Gravitational Wave Backgrounds: A Line Of Sight Approach", Phys. Lett. B771 (2017) 9-12, arXiv:1609.08168 [astro-ph.Co].

[81] N. Bartolo, D. Bertacca, S. Matarrese, M. Peloso, A. Ricciardone, A. Riotto, and G. Tasinato, "Anisotropies and non-Gaussianity of the Cosmological Gravitational Wave Background", arXiv:1908.00527 [astro-ph.C0].

[82] G. Cusin, C. Pitrou, and J.-P. Uzan, "Anisotropy of the astrophysical gravitational wave background: Analytic expression of the angular power spectrum and correlation with cosmological observations", Phys. Rev. D96 no. 10, (2017) 103019, arXiv:1704.06184 [astro-ph.C0].

[83] G. Cusin, I. Dvorkin, C. Pitrou, and J.-P. Uzan, "First predictions of the angular power spectrum of the astrophysical gravitational wave background", Phys. Rev. Lett. 120 (2018) 231101, arXiv:1803.03236 [astro-ph.C0].

[84] G. Cusin, C. Pitrou, and J.-P. Uzan, "The signal of the gravitational wave background and the angular correlation of its energy density", Phys. Rev. D97 no. 12, (2018) 123527, arXiv:1711.11345 [astro-ph.CO].

[85] A. C. Jenkins and M. Sakellariadou, "Anisotropies in the stochastic gravitational-wave background: Formalism and the cosmic string case", Phys. Rev. D98 no. 6, (2018) 063509, arXiv:1802.06046 [astro-ph.CO].

[86] A. C. Jenkins, M. Sakellariadou, T. Regimbau, and E. Slezak, "Anisotropies in the astrophysical gravitational-wave background: Predictions for the detection of compact binaries by LIGO and Virgo", Phys. Rev. D98 no. 6, (2018) 063501, arXiv:1806.01718 [astro-ph.CO].

[87] A. C. Jenkins, R. O'Shaughnessy, M. Sakellariadou, and D. Wysocki, "Anisotropies in the astrophysical gravitational-wave background: The impact of black hole distributions", Phys. Rev. Lett. 122 no. 11, (2019) 111101, arXiv:1810.13435 [astro-ph.C0].

[88] G. Cusin, I. Dvorkin, C. Pitrou, and J.-P. Uzan, "Properties of the stochastic astrophysical gravitational wave background: astrophysical sources dependencies", arXiv:1904.07797 [astro-ph.CO].

[89] G. Cusin, I. Dvorkin, C. Pitrou, and J.-P. Uzan, "Stochastic gravitational wave background anisotropies: astrophysical dependencies in the LIGO/Virgo and LISA bands", arXiv:1904.07757 [astro-ph.CO].

[90] A. C. Jenkins and M. Sakellariadou, "Shot noise in the astrophysical gravitational-wave background", arXiv:1902.07719 [astro-ph.CO].

[91] A. C. Jenkins, J. D. Romano, and M. Sakellariadou, "Estimating the angular power spectrum of the gravitational-wave background in the presence of shot noise", arXiv:1907.06642 [astro-ph.C0].

[92] D. Bertacca, A. Ricciardone, N. Bellomo, A. C. Jenkins, S. Matarrese, A. Raccanelli, T. Regimbau, and M. Sakellariadou, "Projection effects on the observed angular spectrum of the astrophysical stochastic gravitational wave background", arXiv:1909.11627 [astro-ph.C0].

[93] G. Caas-Herrera, O. Contigiani, and V. Vardanyan, "Cross-correlation of the astrophysical gravitational-wave background with galaxy clustering", arXiv:1910.08353 [astro-ph.C0].

[94] N. Seto, S. Kawamura, and T. Nakamura, "Possibility of direct measurement of the acceleration of the universe using $0.1-\mathrm{Hz}$ band laser interferometer gravitational wave antenna in space", Phys. Rev. Lett. 87 (2001) 221103, arXiv:astro-ph/0108011 [astro-ph]. 
[95] B. Kocsis, Z. Frei, Z. Haiman, and K. Menou, "Finding the electromagnetic counterparts of cosmological standard sirens", Astrophys. J. 637 (2006) 27-37, arXiv:astro-ph/0505394 [astro-ph].

[96] C. Bonvin, C. Caprini, R. Sturani, and N. Tamanini, "Effect of matter structure on the gravitational waveform", Phys. Rev. D95 no. 4, (2017) 044029, arXiv:1609.08093 [astro-ph.C0].

[97] C. M. Hirata, D. E. Holz, and C. Cutler, "Reducing the weak lensing noise for the gravitational wave Hubble diagram using the non-Gaussianity of the magnification distribution", Phys. Rev. D81 (2010) 124046, arXiv:1004.3988 [astro-ph.C0].

[98] L. Dai, T. Venumadhav, and K. Sigurdson, "Effect of lensing magnification on the apparent distribution of black hole mergers", Phys. Rev. D95 no. 4, (2017) 044011, arXiv: 1605.09398 [astro-ph.CO].

[99] L. Dai and T. Venumadhav, "On the waveforms of gravitationally lensed gravitational waves", arXiv: 1702.04724 [gr-qc].

[100] S. Mukherjee, B. D. Wandelt, and J. Silk, "Multi-messenger tests of gravity with weakly lensed gravitational waves", arXiv:1908.08950 [astro-ph.CO].

[101] S. Mukherjee, B. D. Wandelt, and J. Silk, "Probing the theory of gravity with gravitational lensing of gravitational waves and galaxy surveys", arXiv:1908.08951 [astro-ph.CO].

[102] T. T. Nakamura, "Gravitational lensing of gravitational waves from inspiraling binaries by a point mass lens", Phys. Rev. Lett. 80 (1998) 1138-1141.

[103] R. Takahashi and T. Nakamura, "Wave effects in gravitational lensing of gravitational waves from chirping binaries", Astrophys. J. 595 (2003) 1039-1051, arXiv:astro-ph/0305055 [astro-ph].

[104] L. Bieri, D. Garfinkle, and N. Yunes, "Gravitational wave memory in $\Lambda$ CDM cosmology", Class. Quant. Grav. 34 no. 21, (2017) 215002, arXiv:1706.02009 [gr-qc].

[105] E. Barausse, V. Cardoso, and P. Pani, "Can environmental effects spoil precision gravitational-wave astrophysics?", Phys. Rev. D89 no. 10, (2014) 104059, arXiv: 1404.7149 [gr-qc].

[106] E. J. Copeland, M. Kopp, A. Padilla, P. M. Saffin and C. Skordis, "Dark energy after GW170817 revisited," Phys. Rev. Lett. 122, no.6, 061301 (2019)

[107] D. Bertacca, A. Raccanelli, N. Bartolo, and S. Matarrese, "Cosmological perturbation effects on gravitational-wave luminosity distance estimates", Phys. Dark Univ. 20 (2018) 32-40, arXiv: 1702.01750 [gr-qc].

[108] F. Schmidt and D. Jeong, "Cosmic Rulers", Phys. Rev. D86 (2012) 083527, arXiv:1204.3625 [astro-ph.CO].

[109] G.-B. Zhao, T. Giannantonio, L. Pogosian, A. Silvestri, D. J. Bacon, K. Koyama, R. C. Nichol, and Y.-S. Song, "Probing modifications of General Relativity using current cosmological observations", Phys. Rev. D81 (2010) 103510, arXiv:1003.0001 [astro-ph.C0].

[110] M. Maggiore, Gravitational Waves. Vol. 1: Theory and Experiments. Oxford Master Series in Physics. Oxford University Press, 2007. http://www . oup. com/uk/catalogue/?ci=9780198570745.

[111] C. M. Will, Theory and Experiment in Gravitational Physics. Cambridge University Press, 2018.

https://www . cambridge.org/academic/subjects/physics/cosmology-relativity-and-gravitation/the 
[112] L. Visinelli, N. Bolis, and S. Vagnozzi, "Brane-world extra dimensions in light of GW170817", Phys. Rev. D97 no. 6, (2018) 064039, arXiv:1711.06628 [gr-qc].

[113] G. Calcagni, S. Kuroyanagi, S. Marsat, M. Sakellariadou, N. Tamanini, and G. Tasinato, "Gravitational-wave luminosity distance in quantum gravity", Phys. Lett. B798 (2019) 135000, arXiv:1904.00384 [gr-qc].

[114] G. Calcagni, S. Kuroyanagi, S. Marsat, M. Sakellariadou, N. Tamanini, and G. Tasinato, "Quantum gravity and gravitational-wave astronomy", JCAP 1910 no. 10, (2019) 012, arXiv: 1907.02489 [gr-qc].

[115] S. R. Dolan, "Geometrical optics for scalar, electromagnetic and gravitational waves on curved spacetime", Int. J. Mod. Phys. D27 (2017) 1843010, arXiv:1806.08617 [gr-qc].

[116] G. Cusin and M. Lagos, "Gravitational wave propagation beyond geometric optics", arXiv:1910.13326 [gr-qc].

[117] D. M. Eardley, D. L. Lee, and A. P. Lightman, "Gravitational-wave observations as a tool for testing relativistic gravity", Phys. Rev. D8 (1973) 3308-3321.

[118] F. Dar, C. De Rham, J. T. Deskins, J. T. Giblin, and A. J. Tolley, "Scalar Gravitational Radiation from Binaries: Vainshtein Mechanism in Time-dependent Systems", arXiv: 1808.02165 [hep-th].

[119] B. P. Abbott, R. Abbott, T. D. Abbott, F. Acernese, K. Ackley, C. Adams, T. Adams, P. Addesso, R. X. Adhikari, V. B. Adya, and et al., "Gravitational Waves and Gamma-Rays from a Binary Neutron Star Merger: GW170817 and GRB 170817A", The Astrophysical Journal 848 no. 2, (Oct, 2017) L13. http://dx.doi.org/10.3847/2041-8213/aa920c.

[120] C. Brans and R. H. Dicke, "Mach's principle and a relativistic theory of gravitation", Phys. Rev. 124 (1961) 925-935. [,142(1961)].

[121] P. Creminelli, M. Lewandowski, G. Tambalo, and F. Vernizzi, "Gravitational Wave Decay into Dark Energy", JCAP 1812 no. 12, (2018) 025, arXiv:1809.03484 [astro-ph.CO].

[122] P. Creminelli, G. Tambalo, F. Vernizzi, and V. Yingcharoenrat, "Resonant Decay of Gravitational Waves into Dark Energy", JCAP 1910 no. 10, (2019) 072, arXiv:1906.07015 [gr-qc].

[123] P. Creminelli, G. Tambalo, F. Vernizzi, and V. Yingcharoenrat, "Dark-Energy Instabilities induced by Gravitational Waves", arXiv:1910.14035 [gr-qc].

[124] D. Jeong, F. Schmidt, and C. M. Hirata, "Large-scale clustering of galaxies in general relativity", Physical Review D 85 no. 2, (Jan, 2012). http://dx. doi.org/10.1103/PhysRevD.85.023504.

[125] C. Dalang, P. Fleury and L. Lombriser, "Horndeski gravity and standard sirens", Physical Review D 102, no. 4, (Aug, 2020), arXiv:1912.06117 [gr-qc]. 